\title{
A low-voltage silicon condenser microphone for hearing instrument applications
}

\author{
Rombach, Pirmin; Müllenborn, Matthias; Klein, Udo; Nielsen, Lis; Frehoff, Roger
}

Published in:

Acoustical Society of America. Journal

Link to article, DOI:

10.1121/1.424815

Publication date:

1999

Document Version

Publisher's PDF, also known as Version of record

Link back to DTU Orbit

Citation (APA):

Rombach, P., Müllenborn, M., Klein, U., Nielsen, L., \& Frehoff, R. (1999). A low-voltage silicon condenser microphone for hearing instrument applications. Acoustical Society of America. Journal, 105(2), 997-997. https://doi.org/10.1121/1.424815

\section{General rights}

Copyright and moral rights for the publications made accessible in the public portal are retained by the authors and/or other copyright owners and it is a condition of accessing publications that users recognise and abide by the legal requirements associated with these rights.

- Users may download and print one copy of any publication from the public portal for the purpose of private study or research.

- You may not further distribute the material or use it for any profit-making activity or commercial gain

- You may freely distribute the URL identifying the publication in the public portal

If you believe that this document breaches copyright please contact us providing details, and we will remove access to the work immediately and investigate your claim. 
Posters from various technical sessions remain on display in the Poster Gallery.

Posters from sessions which contain both lecture and poster presentations will be attended by the authors as listed below.

$\begin{array}{ll}\text { 1pNSb12 } & \text { Weise, Volkmar } \\ \text { 1pNSb13 } & \text { Jiricek, Ondrej } \\ \text { 1pPAb12 } & \text { Niederdraenk, Torsten } \\ \text { 1pPAc12 } & \text { Menguy, Ludovic } \\ \text { 2pNSa12 } & \text { Peters, Juergen }\end{array}$

$$
\text { 10:00-12:00 }
$$

Rotational noise generation in peripheral fans

Experiences with attenuation of low frequency noise in duct

A correlation-measuring-system for ultrasonic NDT using maximum length sequences

Non-linear effect of The inertia of The fluid on acoustic streaming in cylindrical guides Prediction of railway induced vibration by means of transfer functions

Also, the following poster sessions are scheduled:

Poster Session 2aMU (in MA141)

Poster Session $2 \mathrm{aPPb}$

TUESDAY MORNING, 16 MARCH 1999

ROOM H105, 7:55 A.M. TO 12:20 P.M.

\title{
Session 2aAAa
}

\section{Architectural Acoustics: Modeling of Halls; Halls with Special Features}

\author{
Jürgen Meyer, Cochair \\ Physkalisch-Technische Bundesanstalt, Bundesalle 100, 38116 Braunschweig, Germany \\ Leo L. Beranek, Cochair \\ 975 Memorial Drive, Suite 804, Cambridge, Massachusetts 02138-5755, USA
}

Chair's Introduction-7:55

Invited Papers

8:00

2aAAa1. Room acoustic simulation in the planning of concert halls and opera houses. Eckard Mommertz (Müller-BBM, Robert-Koch-Straße 11, D-82152 Planegg, Germany)

In room acoustic planning and design of concert halls and opera houses, computerized prediction of sound transmission gains an increasing significance. The room acoustic simulation may give a more reliable prediction of the room acoustic conditions to be expected. Furthermore, the visualized and auralized results have a positive effect on the communication between acoustician and architect. In this paper, the requirements as well as present possibilities and limitations of room acoustic prediction in practice are described by means of exemplary current building projects. Some important aspects such as coupled volumes, diffuse wall reflections, curved surfaces, or grazing sound transmission above the audience are discussed.

8:20

2aAAa2. Scale model experiences in concert and opera hall design. Takayuki Hidaka (Takenaka R\&D Inst., 1-5-1, Otsuka, Inzai, Chiba, 270-1395 Japan, hidaka.takayuki@takenaka.co.jp)

Scale model experiments were executed for two concert halls and two opera houses which recently opened in Tokyo. Based on the geometrical acoustic analysis by using CAD model, effective room shapes were studied so as to minimize the extra labor at the scale model test. Then, 1:10 scale models made of wood and proper acoustical materials were constructed. Up-to-date digital signal processing technique was applied in order to improve the accuracy of the experiment. Also, several new ideas concerning the sound source, receivers, scale modeled seatings, and how to calibrate the air absorption were introduced, intended for the precise prediction of the room acoustical parameters. Thus, monaural parameters RT, EDT, C80, D, G, and ST, and binaural parameter IACC were measured in the scale models, and they were compared with those measured in real halls. The accuracy and problems of this method were discussed with the acoustical data found here. 
2aAAa3. Recent multi-purpose hall designs in Denmark with physically variable acoustics. Anders Chr. Gade (Dept. of Acoust. Technol., Tech. Univ. of Denmark, Bldg. 352, DK 2800 Lyngby, Denmark, gade@ dat.dtu.dk) and Niels Vilhelm Jordan (Jordan Akustik, Gevninge, Denmark)

This paper describes two recent Danish multi-purpose hall designs, in which acoustic variability has been accomplished in different ways: through variable absorption and variable volume, respectively. The different approaches were chosen in order to insure that not only reverberation time $(T)$ and so clarity $(C)$, but also the strength parameter $(G)$ could be varied in a favorable direction in view of the prescribed uses of the halls. The hall with variable absorption is the assembly hall in the new extension to the Royal Library in Copenhagen to open in fall 1999. This hall will seat 600 people and be used for chamber music concerts, symphony orchestra rehearsals, and amplified speech. For the latter two functions, a reduction in $G$ along with reduction in $T$ was considered favorable. The hall with variable volume is the recently opened Esbjerg Musikhus seating 1100 and equipped with a full stage house. This hall is used for symphonic concerts and musicals as well as drama and conferences. In this hall, maintaining or even increasing $G$ along with a reduction in $T$ is advantageous, especially in the drama theatre mode. The paper will describe the design of these two halls along with acoustic measurement results.

\section{9:00}

2aAAa4. Variable-volume/coupled-volume response at Sala Julio Prestes. Christopher N. Blair (Jaffe Holden Scarbrough Acoustics, 114A Washington St., Norwalk, CT 06854) and José Augusto Nepomuceno (Acústica \& Sônica, 05416-011 São Paulo-SP, Brazil)

Sala Julio Prestes (opening in early 1999) is an adaptive conversion of a railway station in São Paulo, Brazil into a traditional "shoe-box" concert hall. A unique feature of this 1600-seat room is the ceiling, formed in 15 large, tightly spaced, coffered panels. The nine central panels can be raised or lowered independently, while the six edge panels move in symmetric pairs. When the ceiling pieces move together, the room volume can be incrementally adjusted, from a minimum of approximately $12000 \mathrm{~m}^{3}$ to a maximum of about $28000 \mathrm{~m}^{3}$. When the pieces are placed at different levels, various coupled-volume conditions between the lower audience chamber and the volume above the ceiling are also possible. The coupled response is further tunable by provision of absorptive banners which can be deployed in the space above the ceiling panels. Acoustic measurements and binaural recordings of orchestral excerpts in this room with an audience are planned for late 1998 and early 1999. This paper presents preliminary results of these efforts, including recorded musical examples played with various ceiling configurations.

\section{9:20}

2aAAa5. Links between concert hall geometry, objective parameters, and sound quality. Robert Essert (Arup Acoustics, Boston House, 36-38 Fitzroy Square, London W1P 5LL, UK)

For decades, the design of concert halls was driven by considerations of time history alone (T60, C80, ITDG), and as a result, little importance was attached to room geometry. The subjective importance of binaural dissimilarity has been a strong, though often simplistic, influence on recent designs. While listening experience has shown which fundamental room forms sound better than others, computer modeling and statistical analysis have enabled systematic investigation of the degree to which the geometry affects the sound. Using simple parametric models, this study will investigate effects of surface parallelism, concavity, and convexity on spatial and monaural objective acoustical parameters and on essential subjective attributes.

\section{9:40}

2aAAa6. Consistency in acoustic design when building symphonic performance halls. Christopher Jafe, Mark Holden, and Robert Lilkendey (Jaffe Holden Scarbrough Acoustics, Inc., 114A Washington St., Norwalk, CT, cjaffe@jhsacoustics.com)

The acoustic success of all concert hall designs depends on meeting the subjective response of the audience and the performers. Since it is now possible to correlate subjective preferences of listeners and players with quantitative physical acoustic criteria, it is axiomatic that the acoustician should be consistent in setting acoustic environmental criteria for all spaces programmed for symphony performances. This paper describes the application of key physical acoustic criteria related to hall liveness, warmth, and intimacy in the design of rectangular and surround concert halls, multiuse proscenium style performances spaces, and symphonic music pavilions. In addition, the authors will illustrate how the consistent application of these same acoustic criteria can enable the practitioner to develop successful symphony halls and virtual concert facilities utilizing electronic reflected energy systems (ERES). All of the facilities described in this paper incorporated the reflections corelated to listener and performer preferences and were extremely well received by audiences, performers, and the press.

10:00

2aAAa7. Hammerson Hall, The Living Arts Centre in Mississauga, Ontario. Robert Wolff and Damian Doria (Artec Consultants, Inc., 114 W. 26th St., 9th Fl., New York, NY 10001-6812, dd@artec-usa.com)

This paper describes how adjustable acoustics features, effective micro and macro shaping, low background noise levels, and appropriate seat count contribute to a high-quality flexible performance environment. Diagrams, graphs, and images explain the techniques. Hammerson Hall is a concert theater designed to provide suitable acoustic environments for such varied performance media as symphony orchestra, chamber orchestra, recital ensembles, opera, musical theatre, ballet, dance, popular music, speech plays, comedy, lectures, and meetings. The room accommodates 1233 patrons for events when an orchestra is in the pit or on a concert 
platform, but it can quickly accommodate 1315 patrons for events where seats are located on seating wagons on the orchestra pit lift. Hammerson Hall is basically rectangular in shape; however, architectural elements make it appear to be curvilinear. The room also has flexible acoustic absorption and volume. Horizontal balconies and shelves act as reflectors, lateralizing sound energy for acoustic music events.

10:20-10:40 Break

\section{Contributed Papers}

\section{0:40}

2aAAa8. Assessing and optimizing the acoustical parameters of concert halls for Korean traditional music. Kyoungsoo Park, KoengMo Sung (School of Elec. Eng., Seoul Natl. Univ., Seoul, Korea, med@acoustics.snu.ac.kr), and Yeongha Choi (Dept. of Audio Production, Dong-Ah Broadcasting College)

The western music has been studied for many decades in acoustical viewpoint. Many objective parameters are developed for the purpose of assessing the concert halls and optimal values of them were studied. Oriental music, however, and musics of the other cultures have not been studied well yet and neither is Korean traditional music. Although Korean traditional music is somewhat different from western music in sense of the structure of instruments, the style, and the concert halls, something making common people feel the music to be beautiful may have many factors in common. In this paper, a few acoustical parameters of Korean concert halls have been studied by that assumption. Many parameters of traditional concert halls and modern concert halls for Korean traditional music have been measured. The halls were modeled for computer simulation and then varied to find optimal conditions of the halls for Korean traditional music by listening to artificially generated live music in the simulated virtual halls. The method of assessing and finding the optimal condition proposed in this paper may be applied to the other cultural music, too.

\section{1:00}

2aAAa9. Acoustical design of the Walt Disney Concert Hall in Los Angeles. Yasuhisa Toyota (Nagata Acoust., Inc., Hoshino-Bldg. 8F, 5-23-13 Sendagaya, Shibuya-ku, Tokyo 151-0051, Japan, toyota@nagata.co.jp)

A completely new concert hall is scheduled to be opened in the downtown of Los Angeles. The hall was named the Walt Disney Concert Hall, after the late Walt Disney, since the project was started based on the donation of 50 million dollars by Mrs. Disney. The hall was designed as a monopurpose concert hall mainly for orchestral music and is scheduled to be a home hall of the Los Angeles Philharmonic Orchestra and Esa-Pekka Slonen, the Music Director of the orchestra. The hall has approximately 2400 seats and widely spread seating arrangements around the stage like the Berlin Philharmonic hall and the Suntory Hall in Tokyo. Computer simulation studies were carried out to determine the room shape, and acoustic tests with $1 / 10$ scale model were introduced in the final process of the design stage. Construction work is scheduled to start in March 1999, and be completed in 2002. Acoustical design of the hall, especially on its room acoustics and the results of the 1/10 scale model tests, will be reported.

\section{1:20}

2aAAa10. The acoustics evolution of Chicago's orchestra hall: Construction details. Dawn R. Schuette and Scott D. Pfeiffer (Kirkegaard and Assoc., 4910 Main St., Downers Grove, IL 60515)

Chicago's Orchestra Hall is a space that has undergone numerous modifications in its 94-year history. Recent efforts have been the most substantial, producing significant changes. This paper will supplement an invited paper by presenting details of design and construction methods that resulted in the acoustic improvements. Particular issues to be discussed will include construction methods of adding volume to an existing and operating building, details of massive construction used to increase bass response, and techniques of sealing fireproofing, plaster, and concrete within the acoustic volume.

\section{1:40}

2aAAa11. Sopukka, the new experimental stage of the Turku City Theatre. Anssi Ruusuvuori (Akukon Oy Consulting Engineers, Kornetintie 4 A, FIN-00380 Helsinki, Finland, anssi.ruusuvuori@akukon.fi)

Sopukka, the new experimental stage of the City Theatre of Turku, Finland, was built inside the frame of the old small stage. The new stage was designed as a "black box," with movable seating areas for 80 to 150 spectators. The main design goal was flexibility, which would allow as many different staging arrangements as possible. Because of the lack of traditional stage area, the main acoustical design goal was to have an even distribution of sound from any part of the room to any possible seating area. As the ceiling was too high for useful reflections, it had to be absorbed, and the reflecting surfaces were designed as separate elements. The first idea was to hang movable reflecting panels from the ceiling, but the idea was dropped because their moving and adjustment for different settings would have been too cumbersome. Instead, the walls were equipped with fixed diffusers, formed by curved surfaces inside rectangular boxes. These diffusers reflect sound evenly across the room and also back to the performers. The building was completed in March 1997. It has been used for musicals, childrens' plays, and experimental theatre. The acoustics has been considered very favorable.

\section{2:00}

2aAAa12. Excellent acoustics and a low RT? A case study of the recent renovation of the Kennedy Center Concert Hall, Washington, DC. Christopher Jaffe, Paul Scarbrough, Russell Cooper, and Pamela Clements (Jaffe Holden Scarbrough Acoustics, Inc., 114A Washington St., Norwalk, CT 06854)

In October 1997, the renovated Concert Hall at the Kennedy Center reopened to critical acclaim. The hall is designed on the shoebox model, but with an audience of 2759 and a volume of 250 cubic feet per person, its occupied midfrequency reverberation time was below the $2.0 \mathrm{~s}$ that is now considered fundamental to acoustical excellence. The musicians were also having difficulties hearing on stage. The acoustic redesign involved rebuilding the stage with reshaped walls, an acoustical canopy, resonant floor, orchestra risers, seating at stage sides and rear, and refurbished organ. Parterrre walls were added at orchestra level to increase side reflections. Since the ceiling could not be raised, reverberation was enhanced by building small reverberation chambers beside the stage and reducing seat count. Removing parts of the upper side balconies created an area near the stage where the sound field could develop without audience absorption. Changes also included echo control, new seats, and new stage HVAC system. The paper presents data from acoustical measurements and computer models made before and after the renovation, and discusses the combination of acoustical parameters that enables the hall to achieve such high acclaim despite its relatively low reverberation time. 


\title{
Contributed Poster
}

This poster will be on display in the Poster Gallery from Monday to Wednesday, 15-17 March. Author will be at poster from 10:00 a.m. to 12:00 noon on Wednesday, 17 March.

2aAAa13. CAAD—Computer aided architectural and acoustical design. Marius Smigelschi, Alexandru Badescu, and Radu Pana (Inst. of Architecture, Bucharest, Romania)

The computer program "AUDIO" is intended to encourage architects to include (integrate) the room acoustics study in the architectural design from its very beginning, by using in both ways, interactively, the same computer descriptions (AUTOCAD environment) of the room. The main stages of the design are: first: - first architectural variants: - 2-D acoustical comparison, improvement of the room shape in plan, longitudinal, and transversal sections; second: - architectural 3-D model: - 3-D detailed shape analysis, proposed absorbtion characteristics of room bound- aries; third: - architectural details and specifications: - proposals for finishings and materials, taken from the database of the program. In both 2and $3 \mathrm{D}$ stages the program is based on a very performant ray-tracing method. Entry: - room boundaries taken from the architectural model and internally processed (boundary area, volume), audience area, sources (characteristics and location), acoustical processing: - in almost real time, the complete history of impingements (collisions), that is almost statistical, - output: graphic representation of first 2-4 reflections, equal contours on the audience surface of up to ten chosen parameters, (reverberation time, sound level, clarity, spatial impression, lateral efficiency, time gravity point ...), detailed impulse response, reverberation time, and the same ten parameters for any reception point.

TUESDAY MORNING, 16 MARCH 1999

ROOM H2032, 7:55 A.M. TO 12:00 NOON

\section{Session 2aAAb}

\section{Architectural Acoustics and Noise: Modeling of Noise in Work Rooms}

\author{
Murray Hodgson, Cochair \\ Occupational Hygiene Program, University of British Columbia, 2206 East Mall, Vancouver, British Columbia V6T 1Z3, \\ Canada \\ Wolfgang Probst, Cochair \\ ACCON GmbH, Graefelfingerstrasse 133A, D-81375 Munich, Germany
}

Chair's Introduction-7:55

\section{Invited Papers}

\begin{abstract}
8:00
2aAAb1. Prediction of noise in workrooms including extended sources, directivity of radiation, and screening. W. Probst (ACCON GmbH, Graefelfinger Str. 133 A, 81375 Munich, Germany, Wolfgang.Probst@accon.de)

For many years the mirror image method has been used to calculate noise levels in workrooms. Fittings are taken into account by a fitting density defined by Kuttruff and extended to this application by Jovicic. In a research program financed by BAUA Dortmund it was examined how directivity of radiation and screening can be integrated. A removable screen was installed in different halls and it measured the sound distribution in the shadow region, which was produced by a uniformly radiating loudspeaker radiating white noise. Calculation of the same situation by taking into account screening showed that only low orders of reflection are influenced by the screen. This gives the necessary information for a modification of software programs to include screening. Similar to this procedure, a loudspeaker with high directivity was used as source with known emission to measure sound decay curves in workrooms. The same situation was calculated by mirroring the complete directivity of the source. Measured and calculated levels are in good agreement. With these techniques the German standard VDI 3760 for noise calculation in rooms can be improved in the next years.
\end{abstract}

2aAAb2. Experiences with the application of a software for noise prediction in workrooms. Jürgen $H$. Maue (Berufsgenossenschaftliches Institut für Arbeitssicherheit-BIA, Alte Heerstraße 111, D-53757 Sankt Augustin, Germany, J.Maue@hvbg.de)

For a couple of years, the BIA has been using a computer program for the calculation of room acoustic parameters and the prediction of sound pressure levels at the workplace. Experiences gathered with the program in connection with industrial consulting activities are presented and illustrated by examples. The software is based on a calculation method referred to in the German VDI guideline 3760 (image sound source method) requiring a mean degree of complexity in the description of the room. The software comprises a large library, indicating sound absorption coefficients for all tested materials. The program is mainly used for planning room acoustic improvements and new workrooms. Noise prediction in connection with production changes (replacement of machinery, new productions) is also possible. In some cases of hearing damage, the program was used for a retrospective determination of 
noise exposure at former workplace. A comparison of the calculated spatial sound decay curves and the thus-derived room acoustic parameters with the measurement readings showed good correspondence. The development of noise maps and the consequent presentation of the achievable noise reduction by means of concrete measures has become an important instrument for motivating and convincing companies.

8:40

2aAAb3. Is it worth applying sound particle scattering methods to sound propagation in factory halls?-Results and comparisons of some simulations. Uwe M. Stephenson (Fachhochschule Hamburg, Fb Bauingenieurwesen, Hebebrandstr. 1, D-22297 Hamburg, Germany, U.M.Stephenson@t-online.de)

Sound particle simulation, a version of ray tracing, is meanwhile a well-established method in room acoustics. In usual closed rooms, sound propagation is dominated by multiple reflections. In factory halls, the problem arises that a lot of inner sound scattering obstacles (machines, etc.) may practically not be registered in detail. A principal deficiency of ray tracing is the impossibility of taking diffraction into account. Often only a mean level decay per distance is of interest. Therefore, it is a well-founded and -tested semi-analytical approach of using only a mean scattering surface area per volume $q=S /(4 \mathrm{~V})$ and combining that with a simple mirror image source method for rectangular rooms (German guideline VDI 3760). With the sound particle method, this multiple scattering is directly simulated in a Monte Carlo manner. This has several advantages: rooms of arbitrary form, diffuse reflecting surfaces, and inhomogeneous distributions of scattering bodies may be simulated. Meanwhile, on modern PCs, computation times lie in the range of only a few minutes. A disadvantage is the much higher effort of defining the (often uncertain!) input data. Some recent results of simulations of typical setups in factory halls (not computable by common methods) are presented and compared.

9:00

2aAAb4. Modeling of noise in factory workrooms-A practical perspective. David N. Lewis (Unilever Research, Port Sunlight, Wirral L48 6EU, UK, David.N.Lewis@Unilever.com)

It has been known for a considerable time that classical diffuse field theory cannot be applied to workrooms where either of the floor dimensions are greater than approximately five times the ceiling height [H. J. Sabine, Proc. Third Annual Noise Abatement Symposium, Vol. 3, 10 October (1952)]. In such large workrooms there are often many sources of noise and the benefits of alternative noise control stategies are often not obvious. Computerized methods of noise prediction in factory workrooms were first developed in Unilever around 1979. The first major application and validation of the technique was on a project to reduce noise in a manufacturing facility in 1980. The main tool for noise prediction is an in-house package which calculates the combined effect of multiple sources at all points $1 \mathrm{~m}$ apart within the building. Source sound power, directivity, and the propagation curves for the building are input, the latter having been derived from ray tracing predictions, empirical models, or measurement. The technique has been used successfully on numerous projects in the last 20 years and is a valued tool for illustrating the likely benefits of acoustic treatment or defining noise control specifications. An overview of the technique and its application is presented.

9:20

2aAAb5. Modeling of sound fields in enclosed spaces with absorbent room surfaces. Stephen M. Dance and Bridget M. Shield (South Bank Univ., SESD, Borough Rd., London SE1 0AA, UK, dances@sbu.ac.uk)

Commercial computer models are now generally available for the prediction of sound levels in industrial workshops. Four models have been used in this investigation; including RAYNOISE v3.0, RAMSETE v1.3, RAYSCAD+v3.3, and FAME v1.0. These models were validated in rooms with highly absorbent room surfaces using standard and Millington-based absorption coefficients. The rooms included hypothetical, performance, experimental, and industrial spaces. Measurement data were available for the six central octave bands. The results are presented for sound levels and reverberation time, in addition to which the execution time for each model is detailed. It was found that Millington-based absorption coefficients provided consistently more accurate predictions for each type of space than standard absorption coefficients.

9:40

2aAAb6. PlantNoise system for the prediction, visualization, and auralization of industrial noise during computer walkthrough. Murray Hodgson, Nelson Heerema, and Kurtis Halingten (Occupational Hygiene Prog. and Dept. of Mech. Eng., U. B. C., 3rd Fl., 2206 East Mall, Vancouver, BC V6T 1Z3, Canada, hodgson@mech.ubc.ca)

PlantNoise, a novel hardware and software system for predicting, visualizing, and auralizing noise in typical industrial workrooms, is described and demonstrated. It is simple, rapid, accurate, and user-friendly, responding to the needs of industrial practitioners. Input data describing a workroom and its noise sources are input. The workroom floor-plan is visualized, along with the noise sources, a 1-m receiver grid, and a receiver icon. Octave-band and total noise levels, and octave-band reverberation times, are predicted at the receiver location, using new empirical models, and are displayed. The noise is also auralized, using a sound card. The receiver can be "walked-through" the workroom arbitrarily under user control. Visualized and auralized data are updated in real time. Noise contours can be plotted. At any time, the workroom can be modified—for example, to introduce noise-control measures—and the modified noise visualized and auralized. 
2aAAb7. An adaptive beam tracing method for room acoustics prediction. Yiu W. Lam and Ian Drumm (Dept. of Acoust. and Audio Eng., Univ. of Salford, Salford M5 4WT, UK)

A new adaptive beam-tracing algorithm was developed and implemented into a computer model to predict the propagation of sound in enclosed spaces. Unlike previous beam-tracing methods, the model predicts a near perfect geometrical representation of a propagating sound field by adaptively tailoring it to the shape of the reflecting surfaces. Using this adaptive approach, problems such as gaps and overlaps between beams, and the ambiguity of reflection direction when a beam hits more than one surface can be eliminated. In addition, the beam tracing can start with only a small number of beams. Child beams are generated automatically upon each reflection to adapt to the complexity of the room. This method runs faster than traditional beam and ray-tracing methods in the early part and faster than the image method in the later part of the reverberation process. Diffuse reflections are handled by a radiant exchange process and combined with the specular reflections to calculate the impulse response of a room. This model is applied to several spaces with representative workspace shapes and fittings and the result is compared with traditional prediction methods. The suitability of using diffuse reflections to model the effect of factory fittings is investigated.

10:20-10:40 Break

\section{Contributed Papers}

10:40

2aAAb8. Call center as an acoustical challenge. Ernst Joachim Voelker and Sabine Fischer (Inst. for Acoust. and Bldg. Phys., Kiesweg 22, 61440 Oberursel, Germany)

Call centers differ from the standard open plan offices in that telephone calls using headsets lead to special types of activities at the working places. Phone calls with clients produce almost simultaneously an A-weighted speech-noise level of around $52-58 \mathrm{~dB}$ when, for instance, 12 or 18 neighboring working places are occupied. On the other hand, the background noise produced by all persons working is much lower at around $43-45 \mathrm{~dB}$ in well-known open plan offices when quiet work (such as paperwork) is being performed, or when employees are absent. In both cases, there are certain levels of privacy expected. Call centers belong to grade three privacy with a permitted signal-to-noise ratio of around $9 \mathrm{~dB}$. Many room acoustical properties must be considered, such as the falling off of sound levels with increasing distances, avoidance of disturbing sound reflections, short reverberation times, and well-designed sound barriers which must have a soundproofing quality and a high sound absorption. Measurements in existing call centers can be related to the grades of privacy within a certain field of confidence. The same applies to other open plan offices. Artificial background noise such as sound conditioning must be introduced in order to reach the privacy accommodations.

\section{1:00}

2aAAb9. Acoustic quality at workplaces. Lia Kortchmar, Michael Vorländer (Inst. of Tech. Acoust., RWTH Aachen, Templergraben 55, D-52056 Aachen, Germany, lia@akustik.rwth-aachen.de), and Jules G. Slama (PEM-COPPE-UFRJ, Rio de Janeiro, Brazil)

The pursuit of a methodology to elucidate the workplaces issue is a challenge within the area of acoustic quality. The matter involves the search for subjective attributes to be employed on rating acoustic quality judgements. In this search for evaluation strategies, it is appropriate to associate a physical property of the enclosure to a subjective feature. Objective measures offer an intermediate description between design and the subjective effect. The emphasis here is on the objective attributes significant for sound perception. This work looks at some practical experience gained from the connection between experimentally measured values and subjective sensations related to sound quality at workplaces. Information is crossed among the different techniques used for its evaluation. The qualitative features of extremely nondiffuse sound fields are examined by the analyses of the physical properties, objective measures, and subjective quality of various types of rooms. [This work has been carried out at the Institute of Technical Acoustics at the RWTH Aachen University in collaboration with the Federal University of Rio de Janeiro (COPPE/UFRJ).]

\section{1:20}

2aAAb10. Modeling huge sound sources in a room acoustical calculation program. Claus L. Christensen (Dept. of Acoust. Technol., DTU, Bldg. 352, DK-2800 Lyngby, Denmark, clc@dat.dtu.dk)

A room acoustical model capable of modeling point sources, line sources, and surface sources is presented. Line and surface sources are modeled using a special ray-tracing algorithm detecting the radiation pattern of the surfaces of the room. Point sources are modeled using a hybrid calculation method combining this ray-tracing method with image source modeling. With these three source types it is possible to model huge and complex sound sources in industrial environments. Compared to a calculation with only point sources, the use of extended sound sources is shown to improve the agreement with measured data.

\section{1:40-12:00 Discussion}




\title{
Session 2aAB
}

\section{Animal Bioacoustics: Physics of Animal Bioacoustics I}

\author{
James H. Miller, Cochair \\ Department of Ocean Engineering, University of Rhode Island, Narragansett, Rhode Island 02882, USA \\ Christine Erbe, Cochair \\ Institute of Ocean Sciences, 9860 West Saanich Road, Sidney, British Columbia V8L 4B2, Canada
}

Invited Papers

8:00

2aAB1. Insect sounds in agriculture. Robert Hickling (Natl. Ctr. for Phys. Acoust., Univ. of Mississippi, University, MS 38677)

Insects make sounds, either in the course of normal activity (for example, eating or moving about), or to communicate. To investigate activity sounds, it is generally necessary to have a quiet environment, which occurs naturally in grain or soil, and can be created artificially in a soundproof box or anechoic chamber. These sounds can be used to detect pests in agricultural commodities, such as rice weevils in grain, grubs in soil, fruit-fly larvae in fruit, and bollworm in cottom bolls. Examples can be heard on the internet [www.olemiss.edu/ lee/]. A brief discussion is presented of sound transmission in grain and soil. Sound does not appear to be used for communication by larvae. However some grubs in soil may use sound to warn off other grubs from their feeding areas. Acoustic communication appears to be important to ants. Results are presented of sounds made by the black imported fire ant Solenopsis richteri. Examples can be heard on the internet [www.olemiss.edu/ hickling/]. It is shown how ants can make use of the near-field properties of a simple sound source in air. [Work supported by the USDA.]

8:20

2aAB2. Directional hearing in small animals: Tympanal mechanics and evolutionary innovations. Daniel Robert (Inst. for Zoology, Zurich Univ., Winterthurerstr. 190, CH-8057 Zurich, Switzerland)

Tympanal hearing is an evolutionary innovation that occurred at least twice independently in flies (Diptera). Both in Tachinidae and Sarcophagidae fly families, hearing organs evolved that are located on the anterior thorax and that present two thin tympanal membranes rigidly connecting to a pair of mechano-receptive sensory organs. Remarkably, the small interaural distance $(1 \mathrm{~mm})$ constitutes a serious challenge for directional hearing at 3-6 kHz. The mechanical response of these ears to incident random noise was investigated by microscanning laser Doppler vibrometry. Mechanical transfer functions of tympanal displacements show that the ipsiand contralateral tympana vibrate with an unexpectedly large amplitude difference and interaural delay [R. N. Miles et al., J. Acoust. Soc. Am. 98, 3059-3070 (1995)]. The deflection shape analysis shows that in both cases the tympanal membranes are mechanically coupled across the animals midline. However, morphological and biomechanical evidence indicates that the process of mechanical coupling differs between both families, indicating the presence of two alternative and distinct mechanisms for directional sensitivity. This biomechanical and morphological study exemplifies how evolutionary innovation can generate original and specific solutions to the general problem of directional auditory processing. [Work supported by NIH, NSF, and the Swiss Science Foundation.]

8:40

2aAB3. Directional hearing in grasshoppers: Biophysical mechanisms and animal performance. Marc Holderied, Johannes Schul, Dagmar v. Helversen, and Otto v. Helversen (Inst. of Zoology II, Univ. of Erlangen, Staudtstr. 5, 91054 Erlangen, Germany, mholderi@biologie.uni-erlangen.de)

Grasshopper hearing organs are pressure difference receivers: two tympana are connected by air-filled tracheal sacs. Sound reaches a tympanum's outer surface directly and its inner surface through these sacs. Recently, Michelsen and Rohrseitz determined the transfer function of these sacs and developed a biophysical model to predict directional hearing. Tympanal nerve recordings made in Erlangen revealed that this model can predict hearing even for complex stimulus situations with two sound sources emitting pure tones of varying phase and amplitude. Behavioral tests using broadband grasshopper songs showed that a 1-dB intensity difference between two lateral sound sources is sufficient for correct lateralization. It is, however, difficult to predict actual interaural intensity differences for broadband noise by model calculation. A direct method to determine the actual interaural intensity difference necessary for correct lateralization is to decouple the two tympana. For this purpose, light magnetomechanical earphones for free-moving grasshoppers were developed. Lateralization performance using earphones was similar to acoustic stimulation. The possible influence of coherence in broadband signals to the model is discussed. 
2aAB4. Middle ear adaptations in an old world amphibian. Peter M. Narins, Alejandro P. Purgue (Dept. of Physiological Sci., Univ. of California, Los Angeles, 405 Hilgard Ave., Los Angeles, CA 90095), and Edwin R. Lewis (Univ. of California, Berkeley, CA 94720)

Each tympanic membrane (TM) of males of the Old World frog Petropedetes parkeri is decorated with a single, prominent, fleshy papilla during the breeding season. The papilla is composed of a nonossified, spongiform tissue that contain fluid-filled canals that terminate at or near the papillar surface. Roughly three-quarters of the papillar mass is located on the outer surface of the TM, while one-quarter is found on the inner TM surface. Injecting sound into the vocal cavity and measuring the vibration response of a point of the tympanic membrane using laser Doppler vibrometry revealed that the papilla vibrates little in response to sound, but clear modes are observed on the remaining portion of the TM. Moreover, the Fourier transform of the impulse response of the male's intact TM exhibits a peak at $\sim 750 \mathrm{~Hz}$, close to the dominant frequency of the male's advertisement call $(780 \mathrm{~Hz})$. This tuning is interpreted as an adaptation for broadcasting of the call from the eardrums of the male. Surgically removing the papilla resulted in a surprising change in the TM response properties. [Work supported by NIDCD Grant Nos. DC-00222 to PMN and DC-00112 to ERL.]

\section{9:20}

2aAB5. The traveling-wave amplifier model of the cochlea adapted to dolphins. Lars N. Andersen (Dept. of Math. Modeling, Bldg. 321, Tech. Univ. of Denmark, 2800 Lyngby, Denmark, nonboe@eivind.imm.dtu.dk) and Whitlow W. L. Au (Hawaii Inst. of Marine Biol., Kailua, HI 96734)

The traveling-wave amplifier (TWA) model of the cochlea [A. Hubbard, Science 259, 68-71 (1993)] has been shown to produce outputs that compare quite well with experimental data. A TWA model with parameters adjusted to fit the physiological properties of the dolphin cochlea was used as part of a sonar signal discrimination system. The system was tested on a cylinder wall thickness discrimination problem. Broadband echoes from cylinders with different wall thicknesses were aligned using a matched filter and envelope detection. The aligned signals were used as inputs to the TWA model and energy from different locations along the cochlea model were computed in time increments. Data were reduced by principal component analysis and finally classified by a neural network. Results from the experiment demonstrate the potential of the framework.

\section{Contributed Papers}

\section{9:40}

2aAB6. An excitation of surface acoustic waves in the ear canal. E. Grigori Evreinov (Lab. for DIIS, Specvuzavtomatika Design Bureau, 44/ 5-13 Lenin St., Rostov-on-Don, Russia, 344038, evreinovg@usa.net)

Usually electrodynamic loudspeakers have a small flexibility of the diaphragm. Therefore, at sound reproduction of low frequencies, a coupling space between the diaphragm and the ear must be hermetic. In a range of high frequencies, distortions are stipulated by the presence of reflected sound waves, and depend on the ear canal's position in the closed sound space. There is a coupled acoustic resonant system which consists of the pinna, the eardrum, and the ear canal. Sound oscillations excited in a cartilaginous part of the outer ear are partly reflected, but an absorbed component is spread through subcutaneous conjunctive tissue toward an attachment place of the tympanic membrane via surface acoustic waves (SAW). The hearing thresholds' data received by use of a special sound conductor which is generating SAW, in a range of frequencies $125-8000$ $\mathrm{Hz}$, did not show statistic distinctions regarding measurings carried out via conventional tonal audiometry. It is supposed that mechanical energy necessary for excitation of auditory sensation with the help of oscillations spreading through the conjunctive tissue of the ear canal is required even less than at the creation of equivalent levels of air pressure because an amplitude of acoustic oscillations is directly attached to the eardrum edge.

\section{0:00-10:20 Break}

\section{$10: 20$}

2aAB7. Sound production in wasps and ants (Hymenoptera). Gunther Tschuch (Inst. f. Zoologie d. Univ., Entwicklungsbiologie, Domplatz 4, D-06099 Halle, Germany, tschuch@zoologie.uni-halle.de) and Denis J. Brothers (Univ. of Natal, Scottsville, Pietermaritzburg 3209, South Africa)

Some species of ants (Formicidae) and stinging wasps (some Sphecidae, Bradynobaenidae, Rhopalosomatidae, and all species of Mutillidae) possess stridulatory organs without resonance structures. The stridulatory organs occur in both sexes between the 3rd and 4th, 4th and 5th, or 5th and 6th abdominal tergites. The sphecid wasp Pseudoplisus phaleratus makes use of all three positions. The main part of the stridulatory organ is an area of 60 to 200 very regular ripples with species-specific ripple distances of 1.2 to $5 \mu \mathrm{m}$. To prevent mechanical destruction of the ripples during sound production, the force is spread over a width of more than $100 \mu \mathrm{m}$. The signals are optimized to serve as warning signals. Frequently wasps and ants present the acoustical signals together with optical (aposematic coloration) and chemical signals (allomones). The potential predators (e.g., lizards, mammals) are phylogenetically very diverse. Therefore the acoustical signals must have a broad frequency spectrum. The mechanisms used for the production of such spectra were investigated by laser vibrometry. The signals were analyzed by cepstrum and by an adaptive optimal-kernel time-frequency representation [D. L. Jones and R. G. Baraniuk, IEEE Trans. Signal Process. 43, 2361-2371 (1995)]. [Work supported by DFG.]

\section{0:40}

2aAB8. The target strength of the northern right whale (Eubalaena glacialis). James H. Miller (Dept. of Ocean Eng., Univ. of Rhode Island, Narragansett, RI 02882), David C. Potter (Woods Hole Oceanogr. Inst., Woods Hole, MA 02543), Thomas Weber, and James Felix (Univ. of Rhode Island, Narragansett, RI 02882)

The greatest cause of anthropogenic mortality in great whales is ship strikes. Avoiding collisions, which are almost exclusively with baleen whales, may be critical to the survival of the northern right whale (Eubalaena glacialis). A potential solution to this problem is the use of an active forward-looking obstacle avoidance sonar. This sonar could alert the ship's crew of the presence of the whales or other obstacles at useful maneuvering ranges. The prediction of these detection ranges depends on the reflectivity of the whales. The focus of this study is the determination of target strength of the right whale. In May and September of 1998, a prototype phased-array sonar was mounted on the bow of a $25-\mathrm{m}$ research vessel to collect these measurements. The sonar used a $\mathrm{CW}$ ping with a frequency of $87 \mathrm{kHz}$, a duration of $2 \mathrm{~ms}$, a source level of $175 \mathrm{~dB}$ re: 1 $\mu \mathrm{Pa}$ at $1 \mathrm{~m}$. The receive array consisted of an $8 \times 8$ element array with BD wavelength spacing. The frequency was chosen to be inaudible to the baleen whales. Target strengths were also collected for humpback whales (Megaptera novaeangliae), and compare well with previously published data. [Work supported by NMFS and NSF.] 
2aAB9. Modeling animal FM tones using a cubic spline interpolation. Etienne Douaze (Dolphin Study Group, Tropical Marine Sci. Inst., 14 Kent Ridge Rd., Singapore 119 223), Eric Delory, John Potter (Tropical Marine Sci. Inst., Singapore 119 223), and Elizabeth Taylor (Tropical Marine Sci. Inst., Singapore 119 223)

The use of FM tones for communication is widespread among birds and mammals. Analysis of these signals by biologists is currently limited to a qualitative visual inspection of spectrograms and manual logging of discrete signal features from a spectrogram window. Human-related measurement errors are usually not considered and a robust numerical model of animal FM tones is lacking. A model should take at least three signal parameters into account: frequency and amplitude modulation, and the harmonic content. In this work a numerical model for animal FM tone fundamentals is presented. A piecewise polynomial interpolation is used to fit bottlenose dolphin (Tursiops truncatus) FM tones, commonly called whistles, from manually logged spectrogram fundamentals. Cubic splines are used to achieve the best visual fit to the signal fundamental by controlling locally the slope and the curvature of the curve fit. A smoothing factor, calculated from an independent experiment on six human subjects, is applied to the interpolation to adjust for human measurement errors. Dolphin tone fundamentals are modeled with a set of 80 coefficients, a number conveniently independent of signal duration because longer dolphin whistles are observed to be associated with a lesser degree of frequency modulation.

\section{1:20}

2aAB10. Physical constraints on blue whale sound production mechanisms derived from matched-field processing. Aaron M. Thode, Gerald L. D'Spain, and William A. Kuperman (Marine Physical Lab., Scripps Inst. of Oceanogr., Univ. of California, La Jolla, CA 92093-0205)

Matched-field processing (MFP) is a technique for estimating the range and depth of an acoustic source using data collected from a multiple hydrophone array. Physical constraints on the sound production mechanism used by blue whales can be established using this method, including a vocalizing animal's depth, depth and range changes during vocalization, source level estimates, and removal of propagation effects from the animal's source signature. Data are presented from a 1996 experiment off the California Channel Islands that contain a $30-\mathrm{min}$. sequence wherein a single individual produces 10 pulses and 22 FM sweeps from distances up to $5 \mathrm{~km}$ away. The animal appears to vocalize at depths between 15 and 25 $\mathrm{m}$ during both types of vocalizations, and swims with horizontal speeds between 2 to $5 \mathrm{~m} / \mathrm{s}$. The source levels generated by a type A call vary from 176-192 dB re:1 $\mu \mathrm{Pa} @ 1 \mathrm{~m}$ over a 10- to 100-Hz band, consistent with previous estimates [W. C. Cummings, J. Acoust. Soc. Am. 50, Pt. 2, p. 1193]. The type B source levels will also be analyzed and discussed. [Work supported by ONR.]
2aAB11. Using broadband humpback whale vocalizations to locate nonvocal whales in shallow water. Nicholas C. Makris, Yi-San Lai (MIT, 77 Massachusetts Ave., Cambridge, MA 02139), and Douglas H. Cato (Defence Sci. and Technol. Organization, Pyrmont, NSW 2009, Australia)

In a previous paper [Makris and Cato, J. Acoust. Soc. Am. 96, 3270 (1994)], it was shown that a vocal member of a humpback whale herd can be used as a source of opportunity to locate nonvocal members with a passive towed array. That analysis employed full-field but narrow-band propagation and scattering models to emphasize the high spatial array gains available. In the present paper, full-field simulations are performed to determine the structure of actual broadband humpback whale vocalizations after scattering from whales in a shallow-water waveguide. The simulations show that the time signature of a whale vocalization is significantly altered during each of the three stages of (1) propagating from vocal to nonvocal whale, (2) scattering from the nonvocal whale, and (3) propagating from the nonvocal whale to a receiver. The large time-bandwidth gains available in humpback vocalizations then cannot be optimally exploited without first modeling broadband propagation and scattering of the whale vocalization for the given waveguide and bistatic geometry. This raises serious questions about whether the humpbacks themselves, who have limited spatial gains over the noise, can actively detect nonvocal herd members with their vocalizations, as was discussed in the above reference.

\section{2:00}

2aAB12. Anthropogenic ocean noise: Negligible or negligent impact? Darlene Ketten (Woods Hole Oceanogr. Inst., Woods Hole, MA and Harvard Med. School, Boston, MA 02114, dketten@whoi.edu) and John R. Potter (Acoust. Res. Lab., NUS, Singapore 117596)

The increasing use of the oceans is accompanied by rising acoustic pollution. From explosive transients to the nearly continuous drone of ships, anthropogenic noise accompanies virtually every human activity in the sea. Anthropogenic noise has the potential for significant impact on marine species, especially marine mammals, yet existing data are insufficient to predict accurately any but the grossest consequences. There are almost no controlled data on how the acoustic environment is changing as a result of human activity. There is also little information on how marine mammals respond physically and behaviorally, either to intense transients or to a long-term background increase in ambient noise. Ignorance and resulting caution in testing potentially harmful acoustic devices may be seriously hampering our learning progress and the development of effective acoustic deterrents that could decrease marine mammal by-catch. This work discusses the current understanding of acoustic trauma and ambient noise characteristics in the context of what is known about marine mammal hearing and its variations. The work focuses on how species vary in their potential for impact and on determining whether acoustically vulnerable species coincide with acoustic "hot spots" in the ocean where Man's activities may damage hearing and disrupt key behaviors. 


\title{
Session 2aAO
}

\section{Acoustical Oceanography: Innovations in Fish and Plankton Acoustics I}

\author{
Kenneth G. Foote, Cochair \\ Institute of Marine Research, P.O. Box 1870, Nordnes, N-5024 Bergen, Norway \\ David A. Demer, Cochair \\ Southwest Fisheries Science Center, P.O. Box 271, La Jolla, California 92038, USA
}

Invited Papers

\begin{abstract}
8:00
2aAO1. A seven-octave-bandwidth echo sounding system for application to fish and zooplankton. Kenneth G. Foote, Tor Knutsen (Inst. of Marine Res., P.O. Box 1870 Nordnes, N-5024 Bergen, Norway, tor@imr.no), Philip R. Atkins, Claire Bongiovanni, David T. I. Francis (Univ. of Birmingham, Birmingham B15 2TT, UK), Peter K. Eriksen, and Tom Mortensen (RESON A/S, DK-3550 Slangerup, Denmark)

A new echo sounder has been designed and built for measuring broadband acoustic scattering signatures of fish and zooplankton (BASS), which is also the name of the underwriting EU MAST-III project. Development of the system is described in terms of four elements: (1) acoustics, consisting of seven nominally octave-bandwidth transducers spanning the frequency range $25 \mathrm{kHz}$ to 3.2 $\mathrm{MHz}$, depth rated to $300 \mathrm{~m}$, (2) electronics, for control of the transmission and echo reception processes, (3) man-machine interface, allowing remote operation of the transducers and electronics by a menu-driven personal computer, and (4) housing, including mounting of transducers on a bracket and packaging of electronics in a proximate pressure vessel. The system is presently configured for use as a vertical sonde, with ship-derived power. Early in situ applications to euphausiids and Norwegian spring-spawning herring observed on cruises in October and December 1998 are described. The issue of calibration is addressed. Supporting work on modeling the backscattering cross section of marine organisms based on measurement of their morphology and physical properties, and on measuring the same cross section ex situ in a so-called mesocosm of approximate volume 100 cubic meters, is mentioned. [Work supported by the EU through RTD Contract No. MAS3-CT95-0031.]
\end{abstract}

8:20

2aAO2. A multiple-frequency method for potentially improving the accuracy and precision of in situ target strength measurements. David A. Demer (Southwest Fisheries Sci. Ctr., P.O. Box 271, La Jolla, CA 92038, ddemer@ucsd.edu), Michael A. Soule (Sea Fisheries Res. Inst., Cape Town, South Africa), and Roger P. Hewitt (Southwest Fisheries Sci. Ctr., La Jolla, CA 92038)

The effectiveness of a split-beam echosounder system to reject echoes from unresolvable scatterers, thereby improving the measurements of in situ target strengths (TS) of individuals, is dramatically enhanced by combining synchronized signals from two or more adjacent split-beam transducers of different frequencies. The accuracy and precision of the method was determined through simulations and controlled test tank experiments using multiple standard spheres and 38- and 120-kHz split-beam echosounders. By utilizing the angular positional information from one of the split-beam transducers, additional corresponding TS measurements were shown to be obtainable from a juxtaposed single-beam transducer. Both methods were utilized to extract in situ TS measurements of Antarctic scatterers simultaneously at 38,120, and $200 \mathrm{kHz}$. The ultimate efficiency of the multiple-frequency technique is shown to be limited by phase measurement precision, which in turn is limited by the scattering complexity of targets and the receiver bandwidth. Imprecise phase measurements also result in significant beam-compensation uncertainty in split-beam measurements. Differences in multi-frequency TS measurements provided information about the identity of constituents in a mixed species assemblage. The taxa delineation method has potential, but is limited by compounding measurement uncertainties at the individual frequencies and sparse spectral sampling.

\section{8:40}

2aAO3. The investigation of physiologically controlled resonance scattering data from fish using a new acoustic approach. C. Feuillade (Naval Res. Lab., Stennis Space Center, MS 39529-5004, cf@nrlssc.navy.mil)

Low-frequency acoustic scattering from fish is typically dominated by the swimbladder resonance response. Swimbladders are physically similar to bubbles, and it has been traditionally assumed that, as with bubbles, resonance frequency measurements may be directly used to calculate the swimbladdeer volume. Typically, swimbladder models used to interpret fisheries scattering data, and thus determine fish size and abundance, have adopted this premise. Questions are raised, however, by one set of measurements on depth-adapted Atlantic cod by Sand and Hawkins [J. Exp. Biol. 58, 797-820 (1973)], which indicates resonance frequencies much higher, and with more rapid depth variations, than can be realistically explained using such simple assumptions. They argued that the anomalous resonances they observed were actively controlled by the fish, and not just a passive property of the swimbladders. To investigate this phenomenon, a new model was recently developed which incorporates both viscous and elastic properties of fish flesh as variables influencing swimbladder scattering [J. Acoust. Soc. Am. 103, 3245-3255 (1998)]. This presentation will demonstrate how this model provides a basis for understanding the physiological behavior of the fish, and a starting point for determining which species of fish may be reliably surveyed using the traditional approach. 
2aAO4. Fisheries and plankton acoustics: State of the art and beyond. David N. MacLennan (Marine Lab., P.O. Box 101, Victoria Rd., Aberdeen AB11 8DB, Scotland, MacLennan@marlab.ac.uk)

Acoustical methods are well established as a means of remotely observing aquatic organisms. The range of applications reported in the literature is wide, from studies of isolated animals to populations extending over large areas. The geometric scale of target organisms is similarly huge, from microscopic plankton to the largest of marine mammals. The information required from acoustical investigations may be simple quantities like abundance estimates, or more descriptive output like species identification. In each case, there are different problems to be considered. The multi-disciplinary nature of acoustical techniques is important. Success depends on a combined appreciation of scattering physics, animal physiology, statistics, and sonar technology to mention just some of the contributing fields. The historical context is explained, leading to a critical review of recent developments. To a large extent, the driving force has been new technology, especially the rapid growth of computing power. It is important to ensure that appropriate scientific research is done to achieve the benefits of new technology, in acoustics as in other fields, for a better understanding of the living resources in the sea and fresh waters.

\section{Contributed Papers}

9:20

2aAO5. Acoustic observations of the annual cycle of fish and plankton populations in the Beaufort Sea. Robert Pinkel (Scripps Inst. of Oceanogr., 9500 Gilman Dr., La Jolla, CA 92093-0213)

During the period November 1997 through September 1998, a 160$\mathrm{kHz}$ Doppler sonar was operated at the SHEBA ice camp. During this period, the camp drifted clockwise around the Beaufort Sea. The sonar, constructed at the Marine Physical Laboratory of the Scripps Institution of Oceanography, transmitted coded pulses with an 8-kHz bandwidth and recorded echo intensity, spectral bandwidth, and mean Doppler shift. The fall 1997 data featured very weak return echoes. Distinct hard targets were observed over $\sim 5 \%$ of the profile. These have been tentatively identified as Arctic Cod. With changing season and camp location, both plankton and fish populations have evolved significantly. Algorithms have been implemented to track the discrete hard targets and the underlying continuum planktonic population through the course of the year.

\section{9:40}

2aAO6. Acoustic abundance estimation of midwater animals. Kenneth G. Foote and Ingolf Roettingen (Inst. of Marine Res., P.O. Box 1870 Nordnes, N-5024 Bergen, Norway, ingolf.rottingen@imr.no)

The echo integration method of estimating the abundance of midwater animals, specifically pelagic fish and zooplankton, is reviewed. The roles of the modern scientific echo sounder and complementary postprocessing system are emphasized, but requirements imposed by availability of the target animals to surveying by vertical transducer beam, environment including weather and possible presence of other significant scatterers, and the animal itself, are mentioned. The process of converting acoustic measurements of animal density to abundance over the survey region is illustrated in detail for the case of Norwegian spring-spawning herring (Clupea harengus) when wintering in a fjord system. A simple explanation is given for continued interest in the scattering properties of midwater animals, specifically their backscattering and extinction cross sections. The constant need for positive scatterer identification, as through physical capture or optical registration, or, failing these, acoustic classification, is described. The usefulness of geostatistics in estimating abundance and the variance of the abundance estimate, as well as in quantifying the observed spatial distributional properties of the animal, is mentioned. [Partial support of the EU through RTD Contract Nos. AIR2-CT94-1007 and MAS3CT95-0031 is acknowledged.]

\section{0:00-10:20 Break}

10:20

2aA07. Use of acoustics in large tuna trophic-habitat characterization in French Polynesia. Arnaud Bertrand (ORSTOM, BP 70, 29280 Plouzane, France, arnaud.bertrand@orstom.fr)

Tuna have a high metabolic rate, yet they often live in regions characterized by low primary productivity rates. Improving knowledge on tuna-habitat trophic relationship is of main importance for understanding tuna distribution and catchability. Distribution of large tuna food webs, i.e., micronekton, was described in French Polynesia at depths up to $500 \mathrm{~m}$ using acoustics and pelagic trawls. At large scale, acoustics profile response, morphological characterization of acoustic structures, and quantitative descriptors of sound scattering variability were used. Due to water masses advection and oxygen limitation main micronektonic production was localized 8 degrees south of the equatorial upwelling. At a smaller scale, micronekton was classified into seven scattering type: gas-filled swimbladder fish, absent swimbladder fish, gas-bearing invertebrates, cephalopods, fluidlike crustacean, fluidlike jelly-fish, and elastic-shelled invertebrates. Qualitative and quantitative aspects of the scattering structures were also described. Then scattering structure composition and the tuna trophic interest of aggregations was determined.

\section{0:40}

2aAO8. Multi-frequency measures and models of Lake whitefish (Coregonus clupeaformis) backscatter from Lake Michigan. J. Michael Jech, John K. Horne (CILER, Univ. of Michigan, 2205 Commonwealth Blvd., Ann Arbor, MI 48105, jech@glerl.noaa.gov), Lee A. Powell, and James H. Grandt (Univ. of Wisconsin, Madison, WI 53706)

To improve chances for discriminating and identifying fish and zooplankton species, more acoustic information is better. Multifrequency, digital echosounders increase information bandwidth by increasing the number discrete frequencies transmitted and received through multiple transducers. Multifrequency data provide a range of aural perspectives just as colored spotlights accent different components of a painting. Lake whitefish (Coregonus clupeaformis) represent an increasing biomass in Lake Michigan and have a disproportionately large, single-chambered swimbladder relative to the body volume. Frequency-dependent, echo amplitude predictions from a Kirchhoff-ray mode model were compared to in situ backscatter measurements of constrained and free ranging lake whitefish at five discrete frequencies $(38,50,120,200$, and $420 \mathrm{kHz})$. In addition to geometric scattering, backscattering characteristics of whitefish in the resonance region were also modeled. This combination of theoretical model prediction and multifrequency measures allows the quantification of the importance of intraspecies backscatter variability due to fish sizes and behavior. [Work supported by ONR.]

\section{1:00}

2aA09. Status and distribution of the dolphins in the Black Sea-By the acoustically improved line transect sampling technique. Ali $\mathrm{C}$. Gucu (Inst. of Marine Sci., Middle East Tech. Univ., P.O. Box 28, Erdemli, 33731, Icel, Turkey, gucu@ims.metu.edu.tr)

Although dolphins are an endangered species, until 1983 they had been harvested in the Black Sea. Due to severe ecological changes taking place in this sea, the fish stocks experienced a decline within the last decade. The dolphins preying upon fish stocks were adversely affected and their population size was shrunk due to food shortage. But there is an ever growing lobbying on the government for legalizing dolphin fishery, because fishermen believe that the dolphin population has increased since the ban of dolphin fishery, resulting in an increased predation over the fishes 
by dolphins, causing fishery to collapse in 1989 . The aim of this study is to assess dolphin population size within the Turkish EEZ. One of the best techniques to assess the population size of dolphins is the line transect sampling. Being a direct visual census method, the major disadvantage of this technique is the probability of detection, which is not a uniform unity; as far as the eye can see is the basis of the line transect sampling. This disadvantage was eliminated acoustically by using fisheries' acoustic devices, and the sounds of the dolphin recorded by a scientific echosounder were used to detect the existence of the animals on the transect.

\section{1:20}

2aA010. Schools and clusters: Interannual variability in the aggregative behavior of North Sea herring from acoustic surveys. Richard Aukland and David Reid (Marine Lab. Aberdeen, Victoria Rd., P.O. Box 101, Aberdeen AB11 9DB, Scotland, UK, reiddg@marlab.ac.uk)

Commercially exploited pelagic fish species have a strong tendency to aggregate together. Individual fish aggregate into schools of varying sizes and schools will also tend to aggregate into clusters of many schools. If the stock abundance changes, it is expected that the pattern of these aggregations will change. The numbers, sizes, densities of the schools, and clusters may be expected to change with changing stock level. Such changes will in turn influence the conduct of both fishing and survey activities. A knowledge of fish aggregation patterns and the processes involved should improve the reliability of stock monitoring techniques and provide an understanding of the impact of exploitation on dynamically changing populations. Using image processing techniques a database of schools has been developed from data recorded during acoustic surveys for herring in the NW North Sea between 1993-1998. Positional, morphometric, environmental, and energetic descriptors have been computed for each school. The paper will present the results of the analysis of this database, giving particular attention to variation in schooling and clustering patterns, and the spatial distribution of these aggregations, in relation to the changing herring stock size in the period 1993-1998.

\section{1:40}

2aA011. Assessing the relationships between oceanographic parameters and fish distribution: Methodological access and potential problems. Patrick Schneider (Inst. de Ciencias del Mar, Paseo Juan de Borbon, s/n, 08039 Barcelona, Spain, patrick@icm.csic.es)

The management of living resources, such as fish, is depending on the information available on the interrelationships between these resources and other factors-biotic or nonbiotic-prevailing in the system of interest. As far as the impact of oceanographic parameters on the distribution of pelagic fish is concerned, little is known. A profound understanding of distributional patterns of fish, of shifts in distribution, and the interactions between environmental factors and distribution requires at first the simultaneous acquisition of both acoustic and oceanographic data. Haul data and hydroacoustic data, gathered on five small scale surveys in two Mediterranean areas simultaneously with data for temperature, salinity, sigma$t$, and currents, was compared visually and statistically to reveal possible interrelations. As new methods needed to assess the interrelations between environmental and acoustic data are still being developed or adapted from other applications within the scientific community, methodological problems related with this approach are discussed and preliminary results and implications for further investigations and survey designs are presented. Further, new techniques for visualizing survey data are presented and discussed with the objective to open new insights in well-known data. [This work has been financed by the European Commission under Contract No. FAIRGT950139.]

\section{2:00}

2aA012. Observation and extraction of three-dimensional information on fish schools. John Simmonds (Marine Lab., Aberdeen AB11 9DB, Scotland), Francois Gerlotto (ORSTOM, Montpellier, France), Paul Fernandes, and David MacLennan (Marine Lab., Aberdeen AB11 9DB, Scotland)

The paper describes the performance calibration and use of a 90-deg sector scanning sonar for the collection and extraction of information on the 3D structure of fish schools. The equipment, which consists of a 455$\mathrm{kHz}$ 60-beam sector scanning sonar linked to a PC is described briefly. The specific calibration problems of a high-frequency instrument with multiple beams is discussed and calibration data from on-axis and beam shape measurements are presented. The deployment of the instrument for data collection at sea and the data collection methods are described. Examples of the data collected are given. A three-dimensional data processing algorithm is presented along with results of reconstruction from selected schools. The statistical properties of within school data are discussed along with indications of the precision of internal structures that can be evaluated using the sonar. The development of this system is supported by the European research program, AIR. 


\title{
Session 2aEA
}

\section{Engineering Acoustics: Silicon Sensors}

\author{
Ilene J. Busch-Vishniac, Cochair \\ Whiting School of Engineering, Johns Hopkins University, 3400 North Charles Street, Baltimore, Maryland 21218-2694, USA \\ Gerhard M. Sessler, Cochair \\ Institute for Telecommunications and Electroacoustics, Darmstadt University of Technology, Merckstrasse 25, D-64283 \\ Darmstadt, Germany
}

Chair's Introduction-7:55

Invited Papers

2aEA1. Silicon microphones: An overview. Marc Fischer and Gerhard M. Sessler (Inst. for Telecommun. and Electroacoust., Darmstadt Univ. of Technol., Merckstrasse 25, 64283 Darmstadt, Germany)

Over the past few years, silicon micromachining has become a well-established technology. This development was promoted by the expectation that new types of sensors and actuators can be fabricated at low cost. Very early, attempts were made to realize acoustic sensors by the use of micromachining. Only now, the first commercial silicon microphones are available. This paper presents an overview of the technology and discusses different principles to build silicon microphones. There are acoustic sensors, e.g., the capacitive silicon microphone, which are designed by downscaling classical transducers. As opposed to this, there are new types of sensors, making use of novel transduction principles, or of the possibility to integrate active elements on the silicon substrate. The focus of this paper is on the following principles: the capacitive microphone in either single-chip or two-chip design, the piezoelectric microphone with organic or ceramic piezolayer, the piezoresistive microphone utilizing monocrystalline or polycrystalline silicon, the optical microphone based on modulating light propagation in a micromechanical waveguide, and the FET microphone, consisting of an acoustically modulated field-effect transistor. Each transducer will be discussed briefly, including the typical technological process necessary for its design.

8:20

2aEA2. Silicon micromachined microphone chip at Siemens. Alfons Dehé, Thomas Bever, Stephan Schmitt, Sven Michaelis, Hans-Jörg Timme, Ewald Pettenpaul (Siemens AG, Semiconductor Group, HL HF T SNS, Balanstr. 73, D-81617 Munich, Germany), Klaus Oppermann, and Robert Aigner (Siemens AG, D-81739 Munich, Germany)

Applications ranging from hearing aids over communication to noise cancellation open up a high volume market for low-cost, batch producible and reliable microphones. To obey these conditions, a single-chip capacitive microphone has been developed at Siemens, utilizing a modified standard CMOS process with adjacent bulk micromachining. In a first step, the microphone is integrated with a source follower, enabling low output impedance of the signal. The technology allows for the future integration of advanced circuitry. The microphone consists of an acoustically sensitive polycrystalline silicon membrane and a highly perforated back-plate as the counter electrode. To achieve highly sensitive devices, special emphasis was given to the stress of the polycrystalline silicon membrane, which should be slightly tensile. Another key issue during the fabrication and in operation is to prevent stiction of the sensitive membrane. Since the overall chip size is below 3-mm side length, surface mounting in low-cost SMD packages is possible.

8:40

2aEA3. A low-voltage silicon condenser microphone for hearing instrument applications. Pirmin Rombach, Matthias Müllenborn, Udo Klein (Microtronic A/S, Byleddet 12-14, DK-4000 Roskilde, Denmark), Lis Nielsen, and Roger Frehoff (Mikroelektronik Ctr., DTU, DK-2800 Lyngby, Denmark)

Silicon microphones have been the subject of investigations since the early 1980s. Due to their poor performance, no silicon microphone for hearing instrument applications has been commercially available until today. Usually the sensitivity of the electromechanical transducer is too low. Thus the input-related noise of the following preamplifier stage becomes dominant and results in a high equivalent input-related noise. Here a silicon condenser microphone with the potential for hearing instrument applications will be presented. To get the best properties for the different mechanical parts, e.g., membrane and back plate, a dedicated process sequence has been developed. Therefore, circuitry and mechanical parts have to be produced separately and mounted later in a stacking process. The microphone has a $2 \times 2 \mathrm{~mm}^{2}, 0.4 \mu \mathrm{m}$-thick membrane and an air gap of $1.0 \mu \mathrm{m}$. Wafers with different membrane stress have been produced. The microphones have been acoustically, mechanically, and electrically characterized, partly on wafer-scale, and compared with an acoustical/electrical lumped element model. The sensitivity for the low-stress microphones using a $1.5 \mathrm{~V}$ power supply is about $7.0 \mathrm{mV} / \mathrm{Pa}$ and the input related A-weighted noise is $23 \mathrm{~dB}$ SPL, including the preamplifier. Due to a conservative layout, the parasitic capacitance is about $50 \%$. An increase of $2-3 \mathrm{mV} / \mathrm{Pa}$ sensitivity and hence $3 \mathrm{~dB}$ SPL less noise can therefore be achieved by design optimization. 

Technol. Univ., Singapore 639798)

Several miniature silicon capacitive microphones that are newly developed, including a single-chip fabricated condenser microphone and a floating-electrode-electret microphone, and a double-chip fabricated condenser microphone, will be presented. All these microphones take advantage of the corrugated diaphragm technique, which has been proven to be an excellent technique for highly sensitive structures. This technique has greatly improved the performance of the silicon capacitive microphones, especially the sensitivities and noise levels. High reproducibility of the microphone performance has also been achieved as the result of the reduction of the thin-film initial stress effects on the mechanical membranes. Moreover, the corrugation technique makes it possible to integrate easily the electric circuits on the single chip that contains the microphone. Overall design considerations for various microphones will be presented, followed by the finite-element analyses for the mechanical properties of the diaphragms and backplates. Fabrication processes for these microphones are briefly described. Finally, the experimental results are compared with the calculations. The study shows that the single-chip fabricated condenser and electret microphones with integrated preamplifiers have higher sensitivities or greater potentials compared with other capacitive microphones of the same diaphragm sizes, and many promising applications can be expected.

9:20

2aEA5. A novel microelectromechanical system (MEMS) design for an underwater acoustic field sensor. Thomas G. Bifano, Robin O. Cleveland, Debora A. Compton, and Allan D. Pierce (Dept. of Aerosp. and Mech. Eng., Boston Univ., 110 Cummington St., Boston, MA 02115, adp@enga.bu.edu)

A concept for underwater acoustic imaging exploits current technology for constructing MEMS devices from silicon wafers. Anisotropic etching creates frustum-shaped (pyramids with tops cut off) holes in a wafer chip; the bonding of two such chips creates double frustum-shaped holes, each analogous to an hour glass. The front side of each hole is a tapered duct with a baffled open end. The acoustic disturbance generated by an incident plane wave magnifies as the flow converges toward the waist. The back half of the double frustum is covered by a third silicon layer and acts as a Helmholtz resonator, the waist being the neck. The manufacturing process places a thin membrane across the waist which oscillates sychronously with the acoustically induced fluid motion. An appropriate design can achieve fluid velocities at the neck much larger than what would be associated with the incident acoustic wave in free space, and exploratory experiments confirm this. The oscillating membrane is sensed by an optical system, and theoretical extrapolation of the measured displacement amplitude yields a measurement of the amplitude of the incident acoustic wave. [Work supported by Indian Head Divison, Naval Surface Warfare Center, as part of the DARPA Sonoelectronics Program.]

\section{9:40-10:00 Break}

\section{Contributed Papers}

\section{0:00}

2aEA6. Slot silicon microphone: Analytical and experimental study. Zdenek Skvor (CTU Prague, Technicka 2, 166 27, Prague 6, Czech Republic) and Axel Stoffel (FH Furtwangen, Furtwangen im Schwarzwald, Germany)

The diaphragm, as an acoustically active part of the electrostatic silicon transducer, is usually located in the front of the transducer, and the size of the substrate plate determines the entire size of the assembly. It is possible to place on the substrate plate a higher number of sensors and thus obtain a higher sensitivity of the whole system. The increasing number of diaphragms provokes the enlargement of the frontal area of the assembly and the device loses its miniature size. In this contribution a new arrangement is presented of electrostatic or similar pressure sensors with an indirect pressure excitation of their diaphragms and with a small frontal area of the assembly [A. Stoffel and Z. Skvor, Slot microphone, Applic. for U.S. Patent 9,010,032]. Together with an adjacent plate, the substrate plate with diaphragms forms a thin rectangular air-gap with constant or variable cross section and with frontal or frontal and lateral opening. The analytical description is based on the solution of the two-dimensional Helmholtz equation and the transfer function was derived with respect to visco-thermal losses and was experimentally verified.

\section{0:20}

2aEA7. Model measurement of the microphone array. Libor Cerny (Dept. of Radioelectronics, CTU Prague, Technicka 2, 166 27, Prague 6, Czech Republic, CERNYL@FELD.CVUT.CZ)

The use of silicon has allowed the fabrication of microphones with integrated electronic circuitry and the development of the FET microphone. The introduction of lithographic technique has resulted in microphones with very small $\left(1 \mathrm{~mm}^{2}\right)$ diaphragms with specially shaped back- plates. The application of corrugated diaphragms ensures promising future development of silicon microphones. On the basis of this work the arrangement can be prepared for an encapsulation of a miniature microphone chip. Frequency response of the microphone arrays of various types was measured on models in an enlarged scale. It was proven by the numerical results and by the measurements on the model that this manner of encapsulation is possible and advantageous. It was concluded that there is a satisfactory agreement between the analytical description and experimental results of model measurements. The measurement can be transposed to frequency ranges in the ratio of wavelength and dimensions and the damping of modes can be calculated. This problem solution is cheaper than measurement of the miniature microphone chip [Z. Kvor, "Study of the Air-gap and the Influence of the Microphone Position on the Substrate Plate,' Report Copernicus CP 940515 (1997), P. R. Scheeper, Sensors and Actuators A44, 1-11 (1994)].

\section{0:40}

2aEA8. Theoretical and experimental study of the electrostatic transducer with a nonplanar back plate. Jan Skvor (Dept. of Radioelectronics, CTU-Prague, Technicka 2, 166 27, Prague 6, Czech Republic, skvorjan@feld.cvut.cz)

Usually the electrostatic transducer as a microphone or pressure sensor is formed by a circular or rectangular membrane and a planar back electrode with holes. The number, the diameter, and the position of the holes in the back electrode determine the acoustic impedance of the air-gap and especially the damping of the transducer. The aim of this contribution is to show the possibility of the realization of a transducer with a thin diaphragm and a back electrode of convex shape and without holes. The convex shape of the back electrode, because of the nonuniform displacement distribution over the membrane, has a small influence on the transducer sensitivity, but a very high influence on the membrane damping. In 
the contribution is shown the application of the theory of this type of transducer with a circular membrane vibrating in the fundamental mode with approximate parabolic distribution of membrane displacement and a paraboloidal back electrode without the holes. This transducer modification is applicable for classical and silicon micro-mechanical technologies [Z. Skvor and J. Skvor, Proc. 4th Congress on Acoust., SFA, Marseille (1997), Vol. 1, pp. 63-66].

\section{1:00}

2aEA9. A comparison of surface transverse wave propagation on quartz and gallium orthophosphate. Emmanuel Bigler and JeanBernard Briot (Laboratoire de Physique et Métrologie des Oscillateurs Associé à l'Université de Franche-Comté, Besançon, France and 25044 Besancon Cedex, France, bigler@lpmo.univ-fcomte.fr)

Gallium orthophosphate $\left(\mathrm{GaPO}_{4}\right)$ is a piezoelectric material (32-class) with the same symmetry as quartz, but with a higher electromechanical coupling factor and richer possibilities of temperature-compensated acoustic modes in a wide range of temperatures, mainly at high temperatures above $300^{\circ} \mathrm{C}$. Surface transverse waves (STWs) can be generated on both quartz and $\mathrm{GaPO}_{4}$ crystals on Y-rotated plates, so it is interesting to compare both materials on this respect. It is shown that a temperaturecompensated orientation exists on $\mathrm{GaPO}_{4}$ for STWs, but with a lower velocity $(2500 \mathrm{~m} / \mathrm{s})$ than for quartz $(5100 \mathrm{~m} / \mathrm{s})$. Propagation of STWs on $\mathrm{GaPO}_{4}$ under an array of metal strips or grooves is studied and dispersion curves for grooves, aluminum, and gold strips are presented. It is shown that energy trapping near the surface will occur in all cases, allowing STW resonators to be built on $\mathrm{GaPO}_{4}$. The sensitivity of STW devices on $\mathrm{GaPO}_{4}$ to metal thickness and geometrical parameters of electrodes will be investigated. Possible applications to high-temperature devices will be discussed. [This work is supported by CEC, Thomson Microsonics (France) and CNRS (France).]

\section{1:20}

2aEA10. An integrated-optical silicon microphone with a Fabry-Pérot cavity as modulator. Martin Klaiber (Inst. for Telecommunications and Electroacoustics, Darmstadt Univ. of Technology, Merckstr. 25, 64283 Darmstadt, Germany)

A new construction principle for an integrated-optical microphone is presented. It uses a Fabry-Pérot cavity as modulator. This combines the very sensitive interferometric multiple reflection scheme with a direct intensity modulation. The microphone consists of two chips, the carrier chip and the membrane chip. Both are fabricated in silicon using standard semiconductor technology. The membrane chip is mounted on top of the carrier chip, so that both chips together form the Fabry-Pérot cavity. Unmodulated primary light is generated by a laser diode and partially transmitted to the microphone via a $3-\mathrm{dB}$ coupler. A single fiber, which is attached to the bottom side of the carrier chip, connects the coupler and the microphone. The incoming light is modulated in the Fabry-Pérot cavity by the membrane deflection and is reflected back into the fiber. It passes the coupler in reverse direction and is thereby partially transmitted to a receiver device, e.g., a photo diode. The main advantage of this kind of microphone is that a very low inherent noise level $(<30 \mathrm{~dB} / \mathrm{A})$ can be achieved with a relatively simple design.

\section{1:40}

2aEA11. Development of compact fiber-optic microphones. Young C. Cho, Charles K. Gary, Meric Ozcan (NASA Ames Res. Ctr., MS 269-3, Moffett Field, CA 94035-1000, ycho@mail.arc.nasa.gov), and Thomas George (Jet Propulsion Lab., Pasadena, CA 91109)

Advanced pressure sensors have been developed at NASA Ames Research Center, using fiber-optic technology. The development includes: compact size microphone for aeroacoustic measurements in wind tunnels with minimized flow-sensor interaction, and compact size pressure sensors for real-time measurements of flow transition over an airfoil. A prototype pressure sensor was designed and fabricated with a silicon nitride diaphragm mounted on a miniaturized ferrule. The sensor has a 3-mm diameter. Preliminary tests demonstrated excellent performance. The frequency response is steady and uniform within the design frequency limit, 100 to $5000 \mathrm{~Hz}$. Its sensitivity, measured in terms of signal-to-noise ratio, is almost $10 \mathrm{~dB}$ better than the best condenser microphone available in a similar size (0.125-in diameter), and is better than any existing fiber-optic pressure sensor by at least three orders of magnitude. The overall performance of this sensor exceeds the initial expectation. The compact size and light weight of these sensors provide several advantages. The small size could allow tens of hundreds of sensors to be used together for applications such as microphone arrays. The flow-sensor interaction is smaller, providing more accurate measurements of pressure fluctuation of air flows over flight surfaces. 


\title{
Session 2aMU
}

\section{Musical Acoustics: Modeling Versus Measurements of Wind Instruments}

\author{
Avraham Hirschberg, Cochair \\ Eindhoven University of Technology, N-LAAG, P.O. Box 513, 5600 MB Eindhoven, The Netherlands \\ Shigeru Yoshikawa, Cochair \\ Department of Acoustical Design, Kyushu Institute of Design, 4-9-1 Shiobara, Minami-Ku, Fukuoka 815-8540, Japan
}

Chair's Introduction-7:55

Invited Papers

8:00

2aMU1. Mouth tones of flue organ pipes: A control of sound aesthetics. Michele Castellengo (Laboratoire d'Acoustique Musicale, Universite Paris 6, 11 Rue Lourmel 75015 Paris, France)

The quality of the steady sound of organ pipes is determined by pipe geometry and blowing pressure. In contrast to this, the attack transient is controlled by both the voicer and to some extent by the player. The initial part of the transient is related to the so-called "mouth tones," which will be discussed in some detail. On the basis of analyses of the sound production of many organs, the importance of mouth tones during the attack transients of some characteristic stops is shown: Diapason family with initial broadband noises; stopped and chimney pipes with inharmonic "pings;"' Viola stop of the Italian organ with long and intense mouth tones persisting during the stationary sound. As frequency content of mouth tones is directly dependent on the air velocity at the flue, any variation of the pallet opening time in the mechanical action or small uncontrolled fluctuations existing in the wind channel produce many variations in the attack transients. Such variations due to the mouth tones give life to the perceived sound quality of the organ, an instrument where sound production is hardly influenced by the musician.

\section{8:30}

2aMU2. Numerical calculations of woodwind impedances without adequate experimental data: Personal experiences. William J. Strong (Dept. of Phys. and Astron., Brigham Young Univ., Provo, UT 84602)

Some numerical calculations of impedances of an oboe showed relatively good qualitative agreement with experimental data [G. R. Plitnik and W. J. Strong, J. Acoust. Soc. Am. 65, 816-825 (1979)]. With the apparent success of the numerical method, input impedances were calculated for a number of "woodwind-like" structures. On the basis of these calculations a paper was prepared and submitted for publication. Some six months after its submission it was returned with numerous comments regarding errors in its content. A recurring comment from the reviewer was "the digital computer where used alone has contributed directly to most of the difficulties of this paper." Previous to receiving the reviewer's comments the author was using the same numerical method to calculate resonance frequencies of a flute. Difficulty was being experienced in getting reasonable agreement with experimental results. However, after incorporating empirical data into the representation of toneholes rather good results were obtained with the numerical method [W. J. Strong, N. H. Fletcher, and R. K. Silk]. A brief history of these experiences will be presented along with pertinent details from the papers and the reviewer's comments.

9:00

2aMU3. Flow and upstream impedance in wind instruments. P. L. Hoekje (Dept. of Phys. and Astron., Baldwin-Wallace College, 275 Eastland Rd., Berea, OH 44017)

The salient features of the behavior of a wind instrument can be explained by the now-common model of an air column resonator coupled with a flow controller; this paper presents clarifications in that model. The reed flow controller is driven by a pressure difference $p$ and generates a flow $u$; its nonlinearity spreads energy across the spectrum, converting energy from the dc energy source of the player's breath. The instrument air column is characterized by its input impedance $Z_{d}=p_{d} / u_{d}$, where the subscript $d$ denotes the downstream side of the reed. Upstream is the player's airway, characterized by $Z_{u}=p_{u} / u_{u}$ and controlled by the player. The sum $\left(Z_{d}+Z_{u}\right)$ provides feedback to the reed, and when $Z_{u}$ is comparable to $Z_{d}$, the playing qualities of the instrument appear to change. The flow waveform can be estimated from $u_{d}=p_{d} / Z_{d}$, which can then be compared to predictions from dynamical models of the reed motion. To the extent that the reed is incompressible, then $u_{d}=-u_{u}$, and $Z_{u}$ can be estimated from the measurements $\left(Z_{d} p_{u} / p_{d}\right)$. Evidence will also be presented of the nonlinearity of the air column response at loud playing levels. 
2aMU4. Why are historical brass instruments hard to play in tune? D. Murray Campbell (Dept. of Phys. and Astron., Univ. of Edinburgh, Edinburgh EH9 3JZ, UK)

Historical cup-mouthpiece instruments, especially those from the Renaissance and Baroque periods, have the reputation of being harder to play in tune than modern brass instruments. This paper considers what is meant by "playing in tune" from both acoustical and musical standpoints, and surveys acoustical features of some early cup-mouthpiece instruments which may have contributed to their reputation for uncertain intonation. Three different classes of instruments are distinguished. The first includes instruments of fixed acoustical length, such as the natural trumpet; the second includes instruments in which the acoustical length may be lengthened by moving a slide or depressing valves, as on the trombone and the valved trumpet; the third includes cup-mouthpiece instruments in which the acoustical length can be decreased by opening fingerholes, such as the cornetto and the serpent. Different considerations affect intonation in each class of instrument. Experimentally derived input impedance curves, frequency spectra of played notes, and threshold pressure measurements using artificial lips are presented and discussed in the context of intonation sensitivity.

\section{9:40-10:00 Break}

\section{Contributed Posters}

Posters will be on display in Room MA141 on Tuesday, 16 March, from 10:00 a.m. to 12:20 noon to allow contributors an opportunity to see other posters, contributors of odd-numbered papers will be at their posters from 10:00 a.m. to 11:00 a.m. and contributors of even-numbered papers will be at their posters from 11:00 a.m. to 12:00 noon.

2aMU5. Modeling of air-jet-driven instruments: An unstable jet driven by pressure gradient. Seiji Adachi (Dept. of Information Sci. and Technol., Aichi Prefectural Univ., Nagakute, Aichi, 480-1198 Japan)

A new model of the jet deflection is developed to understand the sound production of an organ flue pipe, which is representative of air-jet-driven instruments. This model provides a dynamical equation governing the displacement of a jet, which has the aerodynamical instability and is driven by the acoustic pressure gradient in the direction perpendicular to jet travel. Due to the instability, the growth factor of the deflection becomes exponential, as found in measurements of the jet movement such as hotwire anemometry and flow visualization. The oscillation condition of an experimental E4 organ pipe is examined by considering the phase difference between the jet displacement at the labium and the acoustic pressure at the mouth. The sound is simulated by means of the physical modeling technique. The simulated pipe behavior is compared with that found in an actual pipe. Similarities and differences found in the transition between oscillation regimes are discussed.

2aMU6. Variation of frequency with blowing pressure for an airdriven free reed. James P. Cottingham, Christopher H. Reed, and Michael Busha (Phys. Dept., Coe College, Cedar Rapids, IA 52402, jcotting@coe.edu)

In free reed instruments, an approximately linear decrease of playing frequency with increasing blowing pressure is normally observed. Laboratory measurements on harmonium-type reeds from an American reed organ have shown additionally that at very low pressure there is a small region of increasing frequency with increasing blowing pressure, and at extremely high blowing pressures, the frequency of reed vibration increases rapidly with increasing pressure. Measurements of growth and damping rates confirm the previously reported result [A. O. St. Hilaire, T. A. Wilson, and G. S. Beavers, J. Fluid Mech. 49, 805-815 (1971)] that at low air-flow rates aerodynamic forces add to the mechanical damping of the vibrating reed, but at higher flow rates, the aerodynamic forces contribute negative damping, resulting in self-sustained oscillations. Measurements of reed damping or growth rates have been made over a wide pressure range. Incorporation of these values along with other appropriate parameters in Fletcher's model of reed vibration [N. H. Fletcher, Acustica 43, 63-72 (1979)] permit theoretical calculation of the variation in frequency with pressure for the air-driven free reed. The results of these calculations agree well with experimental data.
2aMU7. Jet and vortex behaviors visualized during the starting transient in organ pipes. Shigeru Yoshikawa (Dept. of Acoust. Design, Kyushu Inst. of Design, Fukuoka, 815-8540 Japan, shig@kyushu-id.ac.jp)

Three types of transient jet behaviors are demonstrated using slowmotion pictures ( 2 to 30 pictures per second) taken by a high-speed digital video camera (1297 or $1440 \mathrm{pps}$ ). (1) The jet issuing from the flue almost always deviates toward the pipe outside and results in a large vortexlike trajectory. When the lower surface of this outwardly curved jet just touches the edge tip, a tiny vortex is likely to be formed beneath the edge. This vortex introduces the first stimulus from the jet to the pipe inside, and in turn significant disturbances are created along the jet. As communicated to the pipe, these disturbances gradually become periodic and wellorganized to build a jet wave. This tiny vortex beneath the edge yields a smooth and fast build-up. (2) A much faster build-up is realized when the jet impinges the edge straightforwardly and divides into the two from the jet center. However, this case is rare. (3) A much slower build-up is caused when the blowing pressure is relatively low and a small vortex tends to stand between the flue and the edge. This stagnant vortex may yield a "two-crest" jet wave prior to the formation of an ordinal "onecrest" jet wave.

2aMU8. Foot resonance in an organ pipe. Claire Segoufin, Benoit Fabre (LAM, UPMC case 161, 4 place Jussieu, 75252 Paris cedex 05, France, segoufin@ccr.jussieu.fr), Vincent Rioux, Munetaka Yokota, Malte Kob, and Mendel Kleiner (Chalmers Univ. of Technol., Gothenburg, S-41279, Sweden)

Pressure measurements in the foot of an organ pipe under playing conditions show acoustical pressure fluctuations induced by the acoustic field in the pipe. Fluctuations of the pressure difference between the foot and the mouth of the instrument results in jet velocity fluctuations that can absorb or produce acoustic energy depending on relative phase with the acoustic pressure in the mouth of the pipe. In most flue organ stops, pipe dimensions indicate that the first pipe resonance and the Helmholtz foot resonance should lie close to each other at least for one pipe in the stop. The present work was triggered by the question of knowing why this does not seem to bother organ builders. Measurements of Helmholtz foot resonances as a function of the driving pressure carried on a pipe with adjustable foot volume are presented. Measurements indicate a damping of the resonance due to the flow through the mouth that can be accounted for by 
an elementary model of the flow through the mouth. Analysis indicates that, unlike recorder playing, the jet velocity guarantees an efficient damping.

2aMU9. Analysis of time-frequency structure of woodwinds' sounds, with usage of discrete wavelet transform. Magdalena Klapper (Dept. of Computer Sci., Univ. of Mining and Metallurgy, Cracow, Poland)

The purpose of this paper is a presentation of analysis results of sounds of woodwinds. The aim of the research was to observe the characteristic properties of the time-frequency structure of particular instruments' sounds, considering the sound register and dynamics. Special attention was paid to the range of tone color registers and possible loudness of sound within them. A trial was undertaken to define the reasons for the division of the instruments' scale in several tone color registers. The mathematical methods used to perform the time-frequency analysis of the instruments' sounds were: mainly multilevel discrete wavelet transform, and also traditional methods: fast Fourier transform and short-time Fourier transform. Discrete wavelet transforms seems to be an especially useful tool for observation of changes of sound parameters. The methods mentioned above vary in distribution of time-frequency analysis. Each of them delivers fragmentary information of the time-frequency structure of the sound; however, they are complementary to one another. Within the framework of the paper a comparison of the analysis results of instrument sounds of quite different properties of time-scale structure are presented.

2aMU10. Attack transients of free reed organ pipes. Jonas Braasch (Inst. of Commun. Acoust., Ruhr Univ., D-44780 Bochum, Germany, braasch@ika.ruhr-uni-bochum.de) and Christian Ahrens (Ruhr Univ., D-44780 Bochum, Germany)

Free reed pipes in organs have fallen out of favor, because they were reputated to have a sluggish attack transient. To test this theory, the starting transients of the "Klarinette $8 \mathrm{ft}$ " pipes were measured on the new Klais organ in the Auditorium Maximum, Bochum. Further measurements to determine the role of the wind pressure were done on the wind chest of the Klais workshop in Bonn. The transients of the free reed pipes were compared to those of bound reed pipes and diapason pipes (measured on the same organ). As the results show, the attack transient of the free reed pipes was indeed slower than that of bound reed pipes, but on the same order of that of diapason pipes. However, in contrast to the free reed pipes, diapason pipes have a noticeable broadband noise preceding the sound. In addition, there is a delay between the onset of the first-order harmonic and the onset of the higher-order harmonic in the starting transient of the free reed pipe. This effect is not usually found in the transient of diapason pipes.

2aMU11. Noise quality of transient sounds: Perception of "hiss" and “cough" in a flue organ pipe. Vincent Rioux, Daniel Västfjäll, Munetaka Yokota, and Mendel Kleiner (Dept. of Appl. Acoust., Chalmers Univ. of Technol., S-41279 Göteborg, Sweden)

According to an expert organ builder, the onomatopoeia "hiss" and "cough" must be distinguished from the well-known general term "chiff" in order to describe the characteristic bursts of noise occuring during the transient of many flue organ pipes. These particular sounds can be adjusted and aligned to a certain aesthetic, but this matter has not yet been much discussed by organ experts. This exploratory study mainly relies on a computer-based listening test which has been carried out on two groups of people (musicians and acousticians). The experiment focused on the participants perception of "hiss" and "cough" and also on their ability to extract the "noisy" part from the harmonic components. Even though the sounds were quite similar, the noise/harmonic separation task was successful for a large number of subjects. This supports the hypothesis of a natural perceptual difference between the noise bursts and the concomitant harmonic setup and also the validity of this decomposition. To differentiate and identify "cough" and "hiss" sounds, however, is a much more elaborate task which requires much experience.

2aMU12. Experimental investigations of wall influences on woodwind instrument sound. Cornelis J. Nederveen (Acacialaan 20, 2641 AC Pijnacker, The Netherlands) and Jean-Pierre Dalmont (Université du Maine, 72085 Le Mans Cedex 9, France)

Influences of wall vibrations and thermal/viscous dissipation at the walls on loudness and sound quality of some woodwind instruments (clarinets, recorders, organ flue pipes) were investigated experimentally. Instruments with various wall thicknesses and wall porosities were studied. Wall vibrations were not found to influence the air oscillations except for a very thin-walled cylindrical organ flue pipe. For this pipe, level increases of 3 $\mathrm{dB}$ and perceptible sound quality changes were observed when the upper part of the pipe was clamped. From input impedance measurements of instruments geometrically identical, but varying in wall porosity, it was found that the quality factor of the resonances could vary by a factor of 2 . The sound level produced by blowing these instruments appeared (at a certain blowing pressure) to be proportional to the real part of the input impedance. The pressure level at which a recorder overblows appeared to increase with the input impedance. Besides wall damping, variations in the bore, which determine the exact positioning of eigenfrequencies, were also found to affect loudness and sound spectrum.

2aMU13. Mechanical response of artificial buzzing lips. John S. Cullen (Dept. of Phys. and Astron., Univ. of Edinburgh, Edinburgh EH9 3JZ, UK), Jöel Gilbert (l’Université du Maine, 72085 Le Mans Cedex 9, France), and D. Murray Campbell (Univ. of Edinburgh, Edinburgh, UK)

Brass instruments sound when the coupling between the acoustical resonator (the instrument) and the mechanical oscillator (the lips) results in a self sustained oscillation. It is the aim of this investigation to characterize experimentally the mechanical response of the lips and to examine its influence on the lip motion observed under near-threshold playing conditions. In this investigation artificial latex rubber lips replace the lips of a human player. Particular emphasis is placed on studying the development of the mechanical response as the embouchure is tightened or as airflow through the lips is introduced. The effect of the acoustical coupling provided by the mouthpiece is investigated. Also presented are playing test measurements, carried out at a level just above the threshold of oscillation. The artificial lips are used to excite resonators of various acoustical lengths; for each resonator the relation of the playing frequency to the nearest lip and resonator resonance frequencies is examined and the measured phase difference between lip opening area and mouthpiece pressure is compared with the phase angles of lip mechanical response and resonator input impedance at the playing frequency. The observed oscillation threshold behavior is compared with that predicted by theoretical physical models.

2aMU14. Reed instruments, from small to large periodic oscillations. Jöel Gilbert, Jean-Pierre Dalmont, and Jean Kergomard (Lab. d'Acoust. de l'Univ. du Maine, UMR CNRS 6613, Ave. Olivier Messiaen, 72085 Le Mans Cedex 9, France)

Reed instruments are physical systems which can be considered as self-sustained oscillators. The "normal operation" of the instrument corresponds to the establishment of a permanent periodic regime. On the one hand, the behavior of the small periodic oscillations in the fixed point (threshold) vicinity is well known: the bifurcation's nature and the oscillation's characteristics depend on both the excitator and the resonator. On the other hand, a theory has been developed for lossless resonators coupled with the excitator: compared to experimental results, this theory is relevant for large amplitude oscillations. The matching between the analytical solutions of the two theories is not instantaneous. The matching is 
studied using the harmonic balance technique with the continuation method coming from known analytical results near the threshold. The bifurcation graphs are constructed for different resonators. The particular resonator with two harmonic resonances is discussed in detail. The role of the octave regime is unexpected: the octave is matched to some of the fundamental regimes. Some resonators (cylinder, lattice of cylinders, cone) are arranged on an artificial mouth designed for single-reed woodwinds. Measurements of periodic regimes are compared to the theoretical results. A movie will be shown.

2aMU15. A virtual reconstruction of the trumpet. Lamberto Tronchin and Alessandro Cocchi (DIENCA-CIARM Viale Risorgimento, 2 40136 Bologna, Italy, tronchin@ciarm.ing.unibo.it)

Virtual reconstructions of musical/instruments have already been analyzed in past years on violins. The "virtual" instruments can be used in subjective listening tests for the evaluation of the sound quality of different instruments, as well as in the restoration of ancient instruments, and for preliminary listening tests on new designed instruments. In this paper, the trumpet is treated as a linear system, characterized by its impulse response. From the IRs measured in different positions in the trumpet, an inverse numeric filter of three trumpets has been obtained, through a new developed technique. From the recording of some original pieces of music, sampled directly inside one of the trumpets, an "anechoic" signal has been obtained by convolution with the inverse filter calculated in the same position. The "anechoic" signal, convolved with the IRs just measured in the other trumpets, produced a signal containing all the acoustic characteristics of the instruments, avoiding all nonacoustic phenomena, and allowed the realization of "virtual" reconstruction of the trumpet. The first results of the listening tests confirm the similarity between the direct acoustic recording and the convolution technique also for the trumpet, as well as already found for the violins.

2aMU16. The wave digital filter brass mouthpiece model. Maarten van Walstijn (Faculty of Music, Univ. of Edinburgh, 12 Nicholson Square, Edinburgh EH8 9DF, Scotland) and Murray Campbell (Univ. of Edinburgh, Edinburgh, Scotland)

Wave digital filter (WDF) techniques are applied to develop an efficient discrete-time two-port mouthpiece model that can be used in traveling-wave based modeling of brass instruments. WDF's were principally developed for simulation of analog networks, and are used here to discretize the classical first-order lumped element mouthpiece model [J. Backus, J. Acoust. Soc. Am. 60, 470-480 (1976)]. The model is segmented into two-port units that are individually mapped to the digital domain using the two-port WDF approach [S. Lawson and A. Mirzai, Wave Digital Filters (Ellis Horwood, London, 1990)]. The realizability of the filter structure is ensured by choosing the port resistances in between the units such that delay-free loops are avoided. The two-port mouthpiece can be directly coupled to models of the lips and leadpipe, although special care must be taken concerning the local characteristic impedance. The WDF mouthpiece model is easy to plug in, its parameters are very intuitive, and it has low computational costs, which makes it particularly suitable for physical modeling with musical sound synthesis purposes. The functioning of the WDF brass mouthpiece model will be further illustrated in a digital wave guide modeling application of a trumpet, wherein all model parameters are derived from measured data.

2aMU17. A computer program for optimization of brass instruments. Part I. Concept, implementation. Wilfried Kausel, Paul Anglmayer, and Gregor Widholm (Inst. f. Wiener Klangstil, Univ. f. Music and Performing Arts in Vienna, Vienna, Austria)

Computer optimization is used to improve characteristics of real world systems. Different strategies are known and have successfully been applied in areas like filter design, circuit synthesis, layout, etc. Common to all optimization methods is the need for mathematical models and target functions evaluating to a single number, reflecting the sum on all deficiencies depending on variables to be varied. In a brass instrument optimizer these variables are representing physical dimensions which later on can be changed by the instrument maker (e.g., by inserting sleeves or modifying the bore). In the target function, differences between actual and desired characteristics like intonation or responsiveness are calculated, weighted, and added. Because these characteristics can only be derived from the instrument's calculated input impedance, physical modeling is required. Systematically and algorithmically, the optimizer approaches the best possible instrument step by step, varying optimization variables. It is directed by the result and tendency of the target function which is evaluated after each step. The brass instrument optimization program introduced here makes computer optimization available for musical instrument manufacturers. Much expert knowledge like defining target functions, specifying optimization variables, and modeling instruments are hidden from the user, making the tool easy enough to work with.

2aMU18. A computer program for optimization of brass instruments. Part II. Applications, practical examples. Paul Anglmayer, Wilfried Kausel, and Gregor Widholm (Inst. f. Wiener Klangstil, Univ. f. Music and Performing Arts in Vienna, Vienna, Austria)

The brass instrument optimization program presented in Part I has been used in a variety of practical cases, demonstrating its power for the improvement of existing brass instruments. In the first and possibly most difficult step, bore lists describing the instrument's geometry have to be obtained. Sometimes data can be imported directly from a CAD system, otherwise mechanical measurements must be done. Next, the dimensions which are to be varied by the program have to be specified. Constraints can be defined. Specific parametric modification rules representing typical working steps of instrument makers (inserting sleeves, narrowing and widening of certain areas) can be applied. Finally, optimization targets have to be defined. Any combination of intonation, impedance magnitude matching, shape, or envelope goals in user-defined frequency intervals is possible. The optimizer will search for the best possible compromise. During the optimization run, all activities can be observed on screen, and target parameters can be adjusted dynamically. In practical examples, real instruments have been optimized and the proposed modifications have been performed by an instrument maker. Measurements and evaluation results made before and after the modifications have been compared. It was shown that the actual performance improvement matches the predicted one well.

2aMU19. Directivity measurement of a singer. Malte Kob and Harald Jers (Inst. of Tech. Acoust., Tech. Univ. Aachen, Templergraben 55, D-52056 Aachen, Germany)

The convenient method of using broadband excitation for fast and accurate evaluation of narrow-band directivity cannot be applied to the human vocal tract. If the voice itself is used as the excitation, the variable properties of the vocal tract change with each differently pronounced phoneme, and therefore a vowel does not yield the same directivity as a consonant. Additionally, the nonstationary character of the voice does not allow for simple measurements with one scanning microphone. In the method described here, the singer stands on a turntable and sings a glissandolike vowel through at least one octave. The sound-pressure level is recorded with two microphones, one as a reference attached to the singer's nose and the other at a 2-m distance. The spectra of both signals are calculated with a sufficiently long FFT, and the levels are subtracted. For each position of the turntable and every angle of elevation, the spectra are stored on disk and postprocessed within a MATLAB environment. Measured directivity plots of several human singers are compared with those of an artificial singer that has been built to reproduce the singing voice accurately. 


\title{
Session 2aNSa
}

\author{
Noise: Community Noise Annoyance \\ Brigitte Schulte-Fortkamp, Cochair
Acoustics/Physics, University of Oldenburg, D-26111 Oldenburg, Germany \\ Sanford Fidell, Cochair \\ BBN Technologies, 21128 Vanowen Street, Canoga Park, California 91303, USA
}

Chair's Introduction-7:55

\author{
B. Berglund, Sweden \\ D. Botteldooren, Belgium \\ S. Fidell, USA \\ T. Gjestland, Norway \\ R. Guski, Germany
}

Panel:

\author{
T. Kaku, Japan \\ P. Lercher, Austria \\ H. Miedema, The Netherlands \\ B. Schulte-Fortkamp, Germany \\ M. Vallet, France
}

Invited Papers

\begin{abstract}
8:00
2aNSa1. Limits of utility of dosage-response analysis for predicting the Prevalence of annoyance. Rainer Guski (Dept. of Psych., Ruhr Universitaet Bochum, D-44780 Bochum, Germany, Rainer.Guski@Ruhr-Uni-Bochum.De)

Dose-response analyses from field studies are essential parts of the information necessary for establishing limits for tolerable noise immissions within the sites (e.g., residential areas with certain immissions) and persons actually studied. But they have their limits when (1) dose-response data are compared between independent studies, (2) annoyance data are extrapolated to other sites, other immission types, levels resp. numbers of noise events, other people, or future times, and (3) the effect of noise abatement programs is predicted. These limitations are presented, using empirical data for illustration purposes. The discussion concentrates (a) on methodological aspects of measuring annoyance, (b) methodological aspects of converting continuous annoyance scales to the percentage of highly annoyed people, and (c) on the contribution of nonacoustic factors to annoyance and to empirical dose-response relationships. It is concluded that dose-response analyses must not be discarded, but their limited utility should be kept in mind.
\end{abstract}

\section{8:20 Panel Discussion chaired by M. Vallet}

\section{8:40}

2aNSa2. Joint noise annoyance: A field evaluation of models concerning road and railway exposure. Peter Lercher (Inst. of Social Medicine, Univ. of Innsbruck, A-6020 Innsbruck, Austria, Peter.Lercher@uibk.ac.at) and Ulrich Widmann (Mller BBM, D-82152 Planegg bei Munchen, Germany)

There is still controversy about how to deal appropriately with a noise exposure situation where two noise sources are of equal importance to an exposed population. Reanalyzing an older German study [Planungsbro Obermeyer (1983)] Ronnebaum et al. (1996) have shown that in a situation where no source is dominant and both sources produce more than $53 \mathrm{~dB}$, Leq, overall annoyance is less than annoyance of the most annoying source. In an ongoing large-noise survey $(N=2000)$ in the Austrian part of the Alps (TYROL) legal requirements force noise ingenieurs to use simple energy summation models in the absence of state of the art alternatives. Selected models [Flindell (1983), DELTA Acoustic \& Vibration (1995)] are evaluated in a complex topographical situation (narrow valley with residential areas on the slopes) where noise annoyance due to railway noise has increased over the last decade in spite of a constant number of train passages. The results of this evaluation will be reported and discussed in terms of its usefulness for environmental risk assessments.

\section{9:00 Panel Discussion chaired by T. Gjestland}

\section{9:20}

2aNSa3. Social survey of the annoyance of low-frequency aircraft ground noise. S. Fidell, L. Silvati, S. Lind, and K. Pearsons (BBN Technologies, 21128 Vanowen St., Canoga Park, CA 91303)

Concerns about the appropriateness of representing low-frequency aircraft ground and near-ground noise in A-weighted units, and about the adequacy of standard interpretive criteria for assessing community response to low-frequency noises, are becoming more common at large civil airports. Residents of a neighborhood adjacent to a busy runway were interviewed to determine the annoyance of runway sideline noise at frequencies below $100 \mathrm{~Hz}$, and of its audible manifestations inside homes. Extensive measurements were made to estimate low-frequency noise contours in the interviewing area, and street addresses of respondents were geo-coded to permit 
assignment of low-frequency noise levels to each household. Residents who were highly annoyed by low-frequency sideline noise were concentrated in areas with maximum sound levels summed in one-third octave bands between $25 \mathrm{and} 80 \mathrm{~Hz}$ (inclusive) that were in excess of 75-80 dB. These levels are consistent with Hubbard's (1980) estimates of low-frequency airborne sound levels capable of inducing secondary emissions in light architectural elements of residences.

\section{9:40 Panel Discussion chaired by R. Guski}

$$
\text { 10:00-10:20 Break }
$$

10:20

2aNSa4. Measurements of noise annoyance: Decisions on appropriate evaluation procedures in field and laboratory settings. Brigitte Schulte-Fortkamp (Acoust./Phys., Univ. of Oldenburg, D-26111 Oldenburg, Germany)

The complicated context of noises that are annoying has to be taken into account to measure annoyance adequately. The measurement has to provide insight information improving the understanding of the complex processes causing the annoyance judgments built up in ranking results, e.g., based on categorical judgments. Another problem will be defined by asking which procedure is more or less sufficient to solve the problems of annoyance measurements or should those measurements be carried out in studies combining both field and laboratory. There is the necessity of a consensus that studies on annoyance have to include the context of activities or interference of activities. Two laboratory studies based on a field study evaluating noise from different sources are carried out in different countries. The results point out the context sensitivity of any judgment as well as the measurement procedure guarantee a detailed characterizing of the noises. The subjective judgments correlate with those parameters relevant for the living situation of each subject. For the laboratory experiments the context is defined by associations evoked by the noises presented. Results of both of the studies strongly demonstrate the need for measurements including the context evaluation and an integrative procedure of field and laboratory studies.

10:40 Panel Discussion chaired by P. Lercher

2aNSa5. Predicting the joint annoyance of multiple noise sources. Birgitta Berglund and Mats E. Nilsson (Inst. of Environ. Medicine, Karolinska Inst. and Dept. of Psych., Stockholm Univ., S-106 91 Stockholm, Sweden)

Noise annoyance is always linked to a complex of environmental noises (noise emission) even if source-specific annoyance is possible to report. Therefore, there is a lack of agreement between total or joint annoyance predicted from source emissions and the actual annoyance reported under field conditions. Models developed from laboratory experiments are commonly pure mathematical constructs based on simple energy summation of totally overlapping sound, whereas the field conditions require yet undeveloped mathematical models that account for perceptual-cognitive integration processes. To be successful in predicting joint annoyance of multiple noise sources, it is necessary that the integration of information derived from noise emission(s) is modeled, rather than simply to sum mathematically noise-source emissions. Important factors for perceptual-cognitive integration processes are kinds of noise sources, their levels, time pattern of joint emissions, discernibility of sounds, and the role these play in the soundscape. Existing models can to varying degree accommodate these factors, but it seems necessary that new models particularly take into account the time pattern of joint emissions as well as the role of sounds in (perceived) soundscapes.

\section{1:20 Panel Discussion chaired by $\mathbf{H}$. Miedema}

\section{1:40}

2aNSa6. Predicting overall reaction to multiple noise sources. Jiro Kaku (Kobayasi Inst. Phys. Res., 3-20-41 Higashimotomati, Kokubunji, Tokyo, 185-0022, Japan), Tohru Kato (Otemon Gakuin Univ., Ibaraki, Osaka, 567-8502, Japan), Sonoko Kuwano (Osaka Univ., Osaka, 565-0871, Japan), and Seiichiro Namba (Takarazuka Univ., Hyogo, 665-0803, Japan)

The validity of models predicting overall human response to complex sound environments, which are constituted of road traffic and railway noises, is discussed using results of psychological experiments carried out both in a laboratory and outdoors, as well as by using results of social surveys. Existing predictive models (energy summation model, independent effects model, response summation model, and so on) were applied to the data. The result shows that the simple energy summation model gives the poorest predicting ability in every case. It suggests that differences of response among different noise sources must be considered. The effective level $D$ of road traffic noise relative to railway noise was calculated using Ollerhead's response summation model as approximately $10 \mathrm{~dB}$ in the laboratory experiment. The $D$ values decreased as compared to the above value in cases of field experiments and social surveys, but it was still clear that the contribution of road traffic noise to the overall judgment was greater than that of railway noise. Judging from the coefficient of determination and standard errors shown in the results, the independent effects model seems to be the most suitable to predict the overall human response to complex sound environments.

12:00 Panel Discussion chaired by D. Botteldooren 


\title{
Session 2aNSb
}

\section{Noise: Computational Aeroacoustics I: Numerical Simulation of Jet and Shear Flow Noise}

\author{
Christopher K. W. Tam, Cochair \\ Department of Mathematics, Florida State University, Tallahassee, Florida 32306-4510, USA \\ Philippe Lafon, Cochair \\ Department of Acoustics and Vibration Mechanics, Electricité de France, 1, Avenue de Général de Gaulle, 92141 Clamart, \\ Cedex, France \\ Invited Papers
}

8:00

2aNSb1. Jet noise simulations using the nonlinear disturbance equations. Philip J. Morris (Dept. of Aerosp. Eng., Penn State Univ., 233P Hammond Bldg., University Park, PA 16802)

Large eddy simulations (LES) of turbulent high Reynolds number flows require significant computational resources if scales in the inertial subrange are to be resolved. However, solutions of the Reynolds-averaged Navier-Stokes (RANS), with an appropriate turbulence model, can provide an efficient and accurate solution for the time-averaged flow. The present formulation recasts the Navier-Stokes equations into equations for the nonlinear perturbations about the RANS approximation to the mean flow. The instantaneous fluctuations are decomposed into a time-averaged component, a resolved large-scale perturbation about this mean, and an unresolved small-scale perturbation. A subgrid scale (SGS) model is used to describe the unresolved scales. The resulting equations are solved on parallel computers using a domain decompositon strategy. Calculations are performed for both circular and rectangular jets. The grid used for the numerical simulations is described. The turbulent fluctuations in the jet, including the time average of the nonlinear perturbations, are presented. Direct simulations of the near and far fields of the jets are given. The jet far field is computed by both direct simulations and with a Ffowcs Williams and Hawkings method. Comparisons are made with available experimental data. [Work supported by NASA Langley Research Center.]

8:30

2aNSb2. Numerical simulation of jet noise: Fundamental issues, progress and outlook. Sanjiva K. Lele (Dept. of Aeronautics and Astronautics and Dept. of Mech. Eng., Stanford Univ., Durand Bldg., Stanford, CA 94305-4035)

High-speed jets remain noisy and loud today. Can high-fidelity numerical simulations provide a new and unique vantage point in future studies of jet-noise and its suppression? What physical and numerical challenges must be overcome in such computational approaches? What features of jet turbulence must be explicitly computed? What is the impact of "sub-grid" scale models, and what precautions must be exercised to avoid "spurious" sources? These fundamental issues will be discussed in the first part of the talk. Recent progress in simulations of high-speed jets, including large eddy simulation (LES), will be presented. The need to examine carefully the interplay between the effects of numerical approximation and physical models being used, will be stressed. The role of reduced-complexity model problems related to jet noise will be discussed and results on sound generation from shock-vortex interaction in a jet mixing layer will be highlighted. Open issues in jet simulations and jet noise modeling will be stressed throughout. This talk includes collaborative work with colleagues Jon Freund, Bendiks-Jan Boersma, Ted Manning, Tim Colonius, and Parviz Moin. [Financial support was (in part) provided by AFOSR under Grant No. F49620-98-1-0355 and NASA under Grant No. NAG 2-1213.]

9:00

2aNSb3. Numerical simulation of jet screech tones. Christopher K. W. Tam (Dept. of Mathematics, Florida State Univ., Tallahassee, FL 32306-4510)

Screech tones are discrete frequency sounds radiated from imperfectly expanded supersonic jets. The tones are generated by a feedback loop driven by the large-scale instability waves of the jet flow. In the plume of an imperfectly expanded jet is a quasiperiodic shock cell structure. Screech tones are produced by the interaction of the instability waves and the shock cells as the former passes through the latter. Although the screech phenomenon has been studied over many years, many of its features are still not understood. Presently, there are accurate tone frequency prediction formulas. However, there is no known way to predict tone intensity. In this study, the screech phenomenon is investigated through numerical simulation using CAA methods. The simulation reproduces all the characteristic features of jet screech, including the staging phenomenon. The computed screech frequencies and intensities are found to compare well with experimental measurements. Numerical results on the effects of jet temperature also compare well with experiments. The favorable agreements indicate that numerical simulation is a valuable tool for investigating the jet screech phenomenon. [Work supported by NASA Lewis Research Center Grant NAG 3-2102.] 
2aNSb4. Inviscid and viscous computations of supersonic jets. San-Yih Lin (Inst. of Aeronautics and Astronautics, Natl. Cheng Kung Univ., 70101, Tainan, Taiwan, ROC)

Numerical investigations of unsteady flow fields and associated sound radiations due to supersonic jets are performed numerically. The axisymmetric Euler and Navier-Stokes equations are solved by two numerical methods, modified Osher-Chakravarthy and WENO. The Reynolds numbers based on the jet diameter and the axial velocity are from 700 to 70000 . For higher Reynolds number flows, the large-eddy equations are used to accurately capture the large-scale vortical structures. The Smagorinsky model is chosen and the Smagorinsky constant is carefully chosen to avoid excessive damping of large-scale fluctuations. The boundary condition treatments are also studied. Two numerical methods are compared with each other to see the effect of dissipation of each method. Kirchhoff's integration method is applied to calculate the far-field noise, with the sound source obtained from the calculated timedependent near field. Details of the flow structures, such as mean flow distributions, shear layer, and the corresponding sound pressure level, are investigated. It is noted that the sound-pressure level obtained by the Euler solver is very high even in the downstream of the jet. Three-dimensional computations are also performed roughly to understand more complicated structures of jet flows.

10:00-10:20 Break

10:20

2aNSb5. Direct numerical simulation of sound generation in turbulent shear flows. Tim Colonius (California Inst. of Technol., Mail Code 104-44, Pasadena, CA 91125)

Direct numerical simulations of turbulent shear flows and their radiated acoustic fields have provided new insights into the mechanisms of sound generation, as well as the efficacy and limitations of the acoustic analogy approach. At present, these computations are expensive, and limited to relatively low Reynolds number and canonical flows. The computational approach consists of using high-order accurate numerical methods, together with accurate and robust nonreflecting and buffer-zone boundary conditions. This talk will focus on the results of computations of sound generated in mixing layers, jets, and open cavity flows. The relationship between linear stability waves in the flow and the radiated acoustic field is examined, in the context of a forced subsonic mixing layer and a fully turbulent supersonic round jet. For the subsonic flow, the acoustic sources can be modeled as wave packets, which radiate a superdirective acoustic field. For the supersonic jet, the directly computed (i.e., nonlinear source) acoustic field is compared to predictions based on radiating stability waves. Finally, the flow/acoustic instabilities in the subsonic flow over an open cavity are examined, and control strategies for reducing the internal acoustic load are explored. [Work supported by NSF (CTS-9501349) and AFOSR (F49620-98-1-0095).]

\section{0:50}

2aNSb6. Vortex dynamics, transition to turbulence, and aeroacoustics in rectangular free jets. Fernando F. Grinstein (Lab. for Computational Phys. and Fluid Dynam., Naval Res. Lab., Washington, DC 20375-5344)

An overview of recent studies of compressible, rectangular, free jets is presented, with special focus on understanding the dynamics and topology of coherent vortical structures controlling the jet development, and identifying major near-jet noise generation mechanisms. Relevant issues of subgrid and supergrid modeling in free-jet numerical simulations are addressed in this context. The vortex dynamics underlying axis-switching and vortex bifurcation phenomena are examined, including: the roles of self-induced vortex-ring deformation, reconnection, braid vortices, aspect ratio (AR), and the transition to turbulence from laminar initial jet conditions. Qualitatively different vorticity geometries characterizing the near field of low-AR, $M<2$, ideally expanded, rectangular jets are demonstrated, involving: (i) self-deforming and (ii) bifurcating vortex rings; interacting ring and rib (braid) vorticesincluding, (iii) single ribs aligned with corner regions $(A R>1)$, and (iv) rib pairs aligned with the corners $(A R=1)$; (v) smaller-scale, elongated, "worm" vortices in the turbulent jet regime. The near-field entrainment of low-AR rectangular jets is shown to be largely determined by the characteristic geometry of the ring-rib interactions; progress in the study of noise-generation mechanisms in low-AR jets is discussed. [Work sponsored by AFOSR, ONR, and the DoD HPC-MP.]

11:20

2aNSb7. Use of linearized Euler equations for subsonic flow noise prediction. Philippe Lafon (Electricité de France, Dept. of Acoust. and Vib. Mech., avenue du Général de Gaulle, 92141 Clamart Cedex, France)

The introduction of efficient numerical techniques like Tam's DRP scheme allows one to develop aeroacoustic modeling based on Euler equation solvers and applied on realistic configurations. It is already clear that the system of Euler equations is the most complete one in order to compute acoustic propagation in fluids, but the way to calculate noise generation with the same equations needs to be established. So, it is necessary to introduce in the propagation calculation some information coming from a previous or a simultaneous flow calculation. One approach is to try to define source terms for the Euler equations as it was set up for Lighthill's or Lilley's equation. Others approaches can rely on the coupling of the two equation systems by domain decomposition or by introducing disturbances linked to flow calculation. The SNGR model belongs to the first category. A source term is defined by analogy with Lilley's equation. A stochastic space time turbulent field is used in order to avoid expensive LES or DNS flow computations. This approach is mainly applied to the calculation of noise radiated by internal flows where the methods based on the classical acoustic analogy do not work easily. 
2aNSb8. Analysis of sound generated by free shear flows using direct numerical simulations. Eldad J. Avital, Kai H. Luo, and Neil D. Sandham (Dept. of Eng., Queen Mary \& Westfield College, Mile End Rd., London E1 4NS, UK)

The sound produced by large-scale structures in free shear flows is investigated using direct numerical simulations (DNS). Both temporal and spatial DNS approaches are investigated, with special attention given to the former due to its lower computational cost. It is shown that the temporal approach can be used to investigate Mach-wave generation but not the general sound emitted by large-scale subsonic sources. Consequently, temporal DNS data of supersonic mixing layers undergoing transition to turbulence are analyzed. Two dominant Mach waves are found, each of which is identified with vortical features in the flow. The spatial DNS approach is then investigated to study the effect of the computational box size on the sound calculation. A large-scale model is used to show that the low-subsonic case is more restrictive in that sense than the supersonic one. The first case is further investigated by calculating the basic radiation emitted by axisymmetric jets, using the compact Lighthill and Mohring-Kambe formulations. Boundary corrections are developed and tested to reduce the box size effect and to improve the agreement between the two formulations. Finally, the effect of the retarded-time variation along the jet is discussed.

TUESDAY MORNING, 16 MARCH 1999

ROOM H2013, 8:35 TO 11:40 A.M.

Session 2aNSc

Noise: Hearing Conservation Program Effectiveness

\author{
Larry H. Royster, Cochair \\ MAE Department, North Carolina State University, Raleigh, North Carolina 27695-7910, USA \\ Laszlo Matéfi, Cochair \\ Department of Occupational Medicine, Swisss Accident National Insurance Fund, \\ Suva, Fluhmattstrasse 1, CH-6002 Lucerne, Switzerland
}

Chair's Introduction-8:35

Invited Papers

8:40

2aNSc1. Hearing conservation for workers in British Columbia and Canada. Christine Harrison (Workers' Compensation Board of British Columbia, Hearing Conservation Section, 6951 Westminster Hwy., Richmond, BC V6B 5L5, Canada, charrison@wcb.ca)

Comprehensive hearing conservation programs for noise-exposed workers in British Columbia have existed since 1978. From 1978 to 1996, a program consisted of engineered noise controls, hearing protection use, and annual hearing tests. In 1996 more statutory components were added: exposure monitoring of workers, posting of noisy work areas, education and training, and annual review of the program. Several administrative aspects of the province-wide program are unique; for example, all hearing tests are recorded on a single, standardized computer form and submitted to the central data base of the Workers' Compensation Board. Industries which have submitted hearing tests include forestry, construction, and manufacturing. The construction program itself is unique in a number of aspects. As of 1998, well over one million audiometric records (each of which includes information regarding occupation, years of noise exposure, threshold values, hearing protection use, nonoccupational noise exposure, age, gender, and more) are in the data base. Analysis of this data has only begun, but interesting trends have been identified. These trends will be presented, with a discussion of methods of program evaluation, as well as an overview of hearing conservation practices across Canada.

9:00

2aNSc2. Distortion product otoacoustic emissions-Valuable method for hearing conservation programs? Laszlo Matéfi (Dept. of Occupational Medicine, Swiss Accident National Insurance Fund, Suva, Fluhmattstrasse 1, CH-6002 Lucerne, Switzerland)

Pure tone audiometry as a screening method for hearing conservation programs has been well established for years. However, it takes a certain time, requires a good cooperation of the patient, and indicates only the damage already caused. Otoacoustic emissions and especially the distortion products have recently been discussed as an alternate method for screening examinations. In the context of this prevention program, some hundred subjects have been examined as usually pure tone audiometry and in addition by measuring the distortion product otoacoustic emissions. The evaluation has been done in the context with the hearing loss measured by pure tone audiometry, with the history of noise exposure, and with the reliability of the distortion product otoacoustic emissions. The present 
results show that there is no reliable association between neither distortion products and hearing loss measured by pure tone audiometry nor between the distortion products and the noise exposure history. The only association found was between important hearing loss measured by pure tone audiometry and absence of distortion products. So, it can be said that distortion product otoacoustic emissions are not a reliable method to be used as screening measurement in hearing conservation programs.

9:20

2aNSc3. Hearing thresholds of pupils and apprentices between the ages of 15-19 years. Karl Körpert (AUVA-HUB, A. Stifterstraße 65, A-1200 Wien, Austria)

Since the early 1990s, several authors have reported on the decreasing hearing ability of young people. The audiograms of the 15-19-year-old men and women show a pronounced dip at 4 and $6 \mathrm{kHz}$, which was not observable a decade before. This fact was observed also in the hearing conservation programs applied to nonoccupationally noise-exposed apprentices in Austria. A commonly suggested reason for that is increased noise exposure in leisure time. To find out if there are differences between apprentices and pupils of the same age, respectively, if information campaigns run during the last years led to an improvement in the hearing status, audiometric investigations were performed in factories and schools in 1992 and 1997. The results of these measurements and relation to leisure time behavior, investigated by questioners, will be reported.

\section{9:40-10:00 Break}

\section{0:00}

2aNSc4. Assessment of hearing conservation program implemented in chosen plants in Poland. Krystyna Pawlas (Inst. of Occupational Medicine and Environ. Health, 13 Koscielna str., 41-200 Sosnowiec, Poland, kape@imp.sosnowiec.pl)

In Poland, as in other industrialized countries, noise is a serious problem of occupational medicine. On the basis of the Polish state law, managers of noisy plants are obliged to implement the hearing conservation program. The program consists of noise measurement, noise reduction by technical means, periodical hearing examination of workers exposed to noise, use of hearing protectors, and training of workers. Nevertheless, $30 \%$ of all new cases of occupational diseases recognized every year in Poland are the noiseinduced hearing loss (NIHL). Most cases of NIHL are recognized in the mining and metallurgical industries. One of the projects previously carried out analyzed the efficiency of medical prophylaxis [J. Grzesik and K. Pawlas, Ochrona Zdrowia Publicznego 96, 516-523 (1985), in Polish]. Hearing conservation programs performed in chosen plants (mines and foundries) were evaluated. Each point of the hearing conservation program was investigated on the basis of the data collected in the plants. Hearing protector devices used by workers were examined, too. The researchers discovered weak points in the activities of health services and occupational hygiene departments as well as of managers and workers.

10:20

2aNSc5. ANSI S12.13: Using audiometric data variability to assess hearing conservation success or failure. Julia D. Royster (Environ. Noise Consultants, Inc., P.O. Box 30698, Raleigh, NC 27622-0698, EFFECTIVE_HCPS@ @ompuserve.com) and Larry H. Royster (North Carolina State Univ., Raleigh, NC 27695-7910)

In 1991, draft standard ANSI S12.13 was published, offering methods for using the variability in audiometric data as an indicator of the effectiveness of hearing conservation programs (HCPs) in protecting noise-exposed personnel from occupational hearing loss. In contrast to methods which require many years of data before hearing thresholds of noise-exposed groups may be compared to control groups, ANSI S12.13 methods may be applied to as few as two successive audiograms. If high variability is found between successive audiograms, it may be due either to unreliable testing (a problem in itself) or to inadequate protection from noise, allowing a temporary and eventually permanent threshold shift to occur. In either case, the evaluator is alerted that the HCP is ineffective. Identifying poor audiometry prevents erroneous decisions from being made based on bad data, both for individuals and for groups. This information enables the evaluator to make needed improvements to the HCP. Examples of analyses will be shown to illustrate patterns of results indicating both procedural problems and insufficient protection. Experience with ANSI S12.13 confirms the value of two simple procedures.

10:40

2aNSc6. An overview of hearing conservation practices in the U.S.A. Larry H. Royster (North Carolina State Univ., P.O. Box 7910, Raleigh, NC 27695-7910) and Julia D. Royster (Environ. Noise Consultants, Inc., P.O. Box 30698, Raleigh, NC 27622-0698, EFFECTIVE_HCPS@compuserve.com)

Federal governmental regulations dealing with the prevention of occupational noise-induced hearing loss began in 1969 with the publication of the Walsh-Healey Act and was extended to general occupations when the U.S. Department of Labor published the OSHA (U.S. Occupational Safety and Health Administration) regulations in 1971. Finally, required procedures for developing an acceptable hearing conservation program were promulgated in 1983 . This presentation will briefly review the history and implementation of the OSHA regulations in U.S. industry. In addition, the present activities by U.S. industry in implementing the OSHA regulations and by OSHA in enforcing them will be reviewed to answer the question: "Is the present implementation of the OSHA noise regulations effective in preventing noise-induced hearing loss in U.S. occupational environments?'” 


\section{Contributed Papers}

11:00

2aNSc7. Research strategies for evaluating effectiveness of industrial hearing loss prevention programs (HLLP). Mary M. Prince, John R. Franks, Carol Merry, and Michael Colligan (Natl. Inst. for Occupational Safety and Health (NIOSH), 4676 Columbia Pkwy., Cincinnati, OH 45226)

NIOSH recommends that hearing loss prevention programs (HLPPs) be implemented for all workers whose unprotected 8-h TWA exposures equal or exceed $85 \mathrm{~dB}$ and that the programs include at least the following components: (1) initial and annual audits of procedures, (2) noise exposure assessment, (3) engineering and administrative control of noise exposures, (4) audiometric surveillance and evaluation, (5) use of hearing protectors (for exposures $>85 \mathrm{~dB}$, regardless of duration), (6) education and motivation, (7) recordkeeping, and (8) program evaluation. All components except program evaluation and routine in-hour audits are generally discussed in the Occupational Safety and Health Administration (OSHA) Hearing Conservation Amendment (HCA). This paper presents research approaches for systematically evaluating OSHA-mandated hearing conservation programs. The intent of such research is to (a) develop methods for evaluating hearing conservation programs that can be used across several industrial sectors and (b) identify ways in which "hearing conservation" can effectively become "hearing prevention" programs. General approaches are presented for each program element and data collection issues are discussed with specific examples of how various methods can been used for program evaluation. The goal of such research strategies is to identify the best predictors of program effectiveness.
11:20

2aNSc8. Assessment of methods for determining field-equivalent sound levels from real-ear and manikin measurements. Dorte Hammershoei and Henrik Moller (Acoust. Lab., Aalborg Univ., Fredrik Bajers Vej 7 B4, DK-9220 Aalborg O, Denmark)

The usual method of measuring noise with a sound-level meter at the position of the exposed person, but with the person absent, is not applicable for sound sources placed close to the ears. For measurement of the noise emission from such sources, the coupling between the sound source and the ear must be similar to that of the real life situation. This can be achieved by using the microphone in real ear technique (the MIRE technique) or the manikin technique as described in the upcoming ISO 11904, parts 1 and 2, respectively. For evaluation and comparison with traditional criteria, the measured ear or manikin sound-pressure levels are subsequently converted into either free-field or diffuse-field equivalent soundpressure levels. The uncertainty of the final result depends on the method used. For the MIRE technique, the uncertainty is mainly related to the statistical uncertainty as determined by the variation between humans and the number of subjects used. For the manikin technique, the uncertainty is mainly related to the deviation of the manikin's acoustical characteristics from those of humans. The paper presents an overview of the methods and examples of practical results.

\section{Contributed Poster}

This poster will be on display in the Poster Gallery from Monday to Wednesday, 15-17 March. The author will be at the poster from 10:00 a.m. to 12:00 noon on Wednesday, 17 March.

2aNSc9. The confounding influence of collapsing the ear canal on audiological assessment of noise-exposed workers. Michel Picard, Chantale Tremblay, and Veronique Dumont (Ecole d'orthophonie et d'audiologie, Univ. de Montreal, Montreal, QC H3C 3J7, Canada, Picardmi@magellan.umontreal.ca)

Collapsing of the ear canal resulting from using supra-aural earphones has been studied in a group of 52 noise-exposed workers. Prevalence and audiometric manifestations of collapsing the ear canal were determined based on comparison of pure-tone air-conduction thresholds obtained when insert earphones are substituted for supra-aural ones in the same individuals. Results indicate that as many as $17 \%$ of candidates showed in one ear or the other, threshold elevations of $6.6 \mathrm{~dB}$ on average at $3 \mathrm{kHz}$, of $8.1 \mathrm{~dB}$ on average at $4 \mathrm{kHz}$, and of $9 \mathrm{~dB}$ on average at $6 \mathrm{kHz}$ as a result of shifting from insert to supra-aural earphones. This finding is of special concern when considering the particular attention paid for the correct positioning of the two types of devices, and the systematic tearing of the tragus in the anterior direction to force the ear canal opening in the positioning of supra-aural earphones. It suggests supra-aural earphones may partially collapse the entrance of the ear canal as a result of the excess pressure applied to the pinna. A model that may help predict the effects of this insidious yet pervasive condition of collapsing ear canals on hearing thresholds of noise-exposed workers will be discussed. 


\title{
Session 2aPAa
}

\section{Physical Acoustics: Thermoacoustics I}

\author{
Cila Herman, Cochair \\ Department of Mechanical Engineering, Johns Hopkins University, 3400 North Charles Street, Baltimore, \\ Maryland 21218, USA \\ Martin Wetzel, Cochair \\ BMW AG, Hufelandstrasse, 80788 Muenchen, Germany
}

Chair's Introduction-7:55

Invited Papers

8:00

2aPAa1. Thermoacoustic natural gas liquefier. Gregory W. Swift (Condensed Matter and Thermal Phys. Group, Los Alamos Natl. Lab., Los Alamos, NM 87545) and John J. Wollan (Cryenco, Inc., Denver, CO 80239)

An experimental thermoacoustic natural-gas liquefier has been built and tested. It comprises a standing-wave thermoacoustic engine and a traveling-wave refrigerator (a pulse-tube refrigerator). The engine and refrigerator share a common resonator, filled with $3 \mathrm{MPa}$ helium gas and operating at $40 \mathrm{~Hz}$. The engine is heated by external combustion of natural gas, while the refrigerator cools and liquefies a methane stream, so the complete system functions as a natural-gas-fired natural-gas liquefier having no moving parts. Both the engine and refrigerator have operated above $20 \%$ of their respective Carnot efficiencies. Compared to other thermoacoustics research hardware, this experimental system is very powerful, with up to $20 \mathrm{~kW}$ of acoustic power delivered from the engine to the resonator and up to $2 \mathrm{~kW}$ of refrigeration power at $130 \mathrm{~K}$ provided by the refrigerator. Nevertheless, much larger systems will be required for commercial success. [Work supported by the Office of Fossil Energy in the U.S. DOE and by Cryenco, Inc.]

8:20

2aPAa2. A pistonless Stirling engine-A new direction in thermoacoustics. Scott N. Backhaus and Gregory W. Swift (Condensed Matter and Thermal Phys. Group, Los Alamos Natl. Lab., Los Alamos, NM 87545)

Due to its thermodynamic reversibility, the Stirling cycle has an inherent advantage in thermal efficiency over the standard, irreversible standing-wave thermoacoustic cycle. The main disadvantage of the Stirling cycle has been the need for high-pressure, sliding piston seals. Building significantly on the ideas of Ceperley [J. Acoust. Soc. Am. 66, 1508 (1979)], a thermoacoustic version of a 1-kW Stirling engine has been constructed and tested. This engine enjoys both the efficiency advantage of the Stirling cycle and the no-moving-parts advantage of thermoacoustics. In this first attempt, experimental data show that the engine has an efficiency above $35 \%$ of the Carnot efficiency, much higher than that of any prior thermoacoustic engine. [Work supported by the Office of Basic Energy Sciences in the U.S. DOE.]

\section{8:40}

2aPAa3. The thermoacoustic oscillator as a musical instrument. Werner Lauterborn and Georg Müller (Drittes Phys. Inst., Univ. Göttingen, Bürgerstr. 42-44, D-37073 Göttingen, Germany)

The air in a tube with a short stack of thin parallel plates inside starts to oscillate when a steep heat gradient is applied. The known device can be considered as an oscillator, more specifically a thermoacoustic oscillator (TAO). It radiates sound when the tube is open to the ambient air. The pitch is determined by the tube length. Thus different pitches can be generated by altering the tube length, allowing the possibility to build musical instruments based on TAOs. As in other instruments with tubes, there are several possibilities to alter the tube length. It may be adjusted by holes that are opened and closed as in a recorder, or by a sliding piston with a hole in it to transmit the sound to the exterior. With the sliding piston, trombonelike sliding sounds can be generated. Examples of musical sounds out of a TAO will be given. A thermoacoustic organ is easily conceivable consisting of TAOs of different lengths played by keys opening and closing the respective TAOs. 
2aPAa4. Measurements of the performance of a thermoacoustic refrigerator prototype. B. L. Minner, J. E. Braun, and L. Mongeau (School of Mech. Eng., Purdue Univ., West Lafayette, IN 47907)

The performance of a thermoacoustic prototype designed for a cooling capacity of $140 \mathrm{~W}$ and operating stack end temperatures of 282 and $306 \mathrm{~K}$ was measured. The device was operated using a 55\%-45\% helium-argon mixture at a mean pressure lower than or equal to 20 bars. The electrodynamic moving coil driver coupled to the half-wavelength resonator was tuned such that the system resonance frequency (i.e., the operating frequency) was around $180 \mathrm{~Hz}$. The stack was made of a coiled polyester film and nylon filament spacers. The secondary system included finnedtube heat exchangers with water as the operating fluid. The power delivered to the driver coil was measured using a power meter. The cooling power was estimated from the water-side temperature drop across the heat exchangers measured using thermopiles, and from the water flow rate. Acoustic pressure, piston acceleration, and temperature measurements were also made at various locations inside the resonator. The measured values were compared with predictions obtained using DeltaE.

\section{9:20}

2aPAa5. Measurements of thermoacoustic functions for unusual stacks. Gabriela Petculescu and Larry A. Wilen (Dept. of Phys. and Astron., Ohio Univ., Athens, OH 45701, wilen@ helios.phy.ohiou.edu)

Following earlier work [G. Petculescu and L. A. Wilen, J. Acoust. Soc. Am. 103, 2841(A) (1998)], a volume-modulation technique is employed to measure directly the complex compressibility of gas in a stack. When a temperature gradient is imposed on the stack, the complex compressibility is sensitive to thermoacoustic effects which can be measured to high precision. These effects depend on both thermal and viscous properties of the gas. Direct measurements of the thermoviscous functions analogous to $F(\lambda)$ and $F\left(\lambda_{T}\right)$ will be presented for unusual geometry stacks, such as conical shapes or stacks constructed from reticulated vitreous carbon (RVC). The behavior of a stack with a sharp temperature gradient approximating a temperature discontinuity will also be described. Time permitting, nonlinear effects may also be discussed. [Work supported by the Office of Naval Research and Ohio University Research and Sponsored Programs.]

\section{9:40}

2aPAa6. Conventional heat exchanger design methods and their applicability to thermoacoustics. Cila Herman (Dept. of Mech. Eng., Johns Hopkins Univ., 3400 N. Charles St., Baltimore, MD 21218, herman@titan.me.jhu.edu)

Heat exchangers (HEX) play an important role in many engineering applications, and well established methods for their modeling and optimization are available. Conventional heat exchangers take advantage of forced convection or phase change to transport thermal energy from the hot to the cold fluid, as opposed to thermoacoustic applications where the mean velocity of the oscillatory flow is zero. Conventional HEX are typically classified according to flow arrangement and type of construction. The most commonly implemented methods for HEX analysis are the logarithmic mean temperature difference (LMTD) method and the number of transport units (NTU) approach. Both methods require a set of input parameters and the analysis yields, for example, the appropriate heat exchanger size, i.e., the heat transfer surface area, and type that achieve the desired fluid outlet temperature. The applicability of conventional heat exchanger design methods to thermoacoustic HEX is explored. HEX design solutions developed for thermoacoustics are analyzed in light of the LMTD and NTU approaches. Key differences and issues requiring further research are addressed in the study. [Work supported by the Office of Naval Research.]
10:20

2aPAa7. Computational analysis of a thermoacoustic refrigerator. Thomas Schneider, Rupert Klein (FB Mathematik \& Informatik, Freie Universität, D-14195 Berlin, Germany), Etienne Besnoin, and Omar M. Knio (Johns Hopkins Univ., Baltimore, MD 21218)

The velocity and temperature fields in an idealized, parallel-plate, thermoacoustic refrigerator are analyzed using a low-Mach-number computational approach. Two-dimensional unsteady calculations of the flow field in the neighborhood of the stack and heat exchangers are performed using: (a) a semi-implicit, finite-volume, Godunov-type, compressible flow solver, and (b) a vorticity-based scheme for stratified flows. The first model is used to address the limiting case of plates with vanishingly small thickness, while the second model is used to analyze finite blockage effects. The predictions of both models are compared to each other and are used to study the effect of heat exchanger properties on the thermal performance of the device. [The work of T.S. and R.K. is supported by DFG. The work of E.B. and O.M.K. is supported by the Office of Naval Research.]

\section{0:40}

2aPAa8. Effects of gas mixture on a thermoacoustic refrigerator driven at high amplitudes. Matthew E. Poese and Steven L. Garrett (Grad. Prog. in Acoust., P.O. Box 30, State College, PA 16804, matt@sabine.acs.psu.edu)

Measurements of a thermoacoustic refrigerator driven at pressure ratios up to $6 \%$, with a He/Ar gas mixture have been reported [M. E. Poese and S. L. Garrett, "Performance measurements of a thermoacoustic refrigerator driven at high amplitudes," Proc. 16th Intl. Congress Acoust. and 135th meeting Acoust. Soc. Am. 2, 809-810 (1998)] and show a $15 \%$ lower heat pumping power at $6 \%$ pressure ratio compared to the fourfold increase in heat pumping power expected by doubling the pressure ratio from $3 \%$. To further characterize the high-amplitude performance, measurements with other gas mixtures $\left(\mathrm{He} / \mathrm{SF}_{6}\right.$ and $\left.\mathrm{He} / \mathrm{Xe}\right)$ were made. These experiments show that while the $\mathrm{He} / \mathrm{Xe}$ mixture enables the machine to operate with a larger COPR than with a He/Ar mixture, the nonlinear cooling power degradation is more severe: $10 \%$ at a $3 \%$ pressure ratio and $20 \%$ at $6 \%$. Experiments with a $\mathrm{He} / \mathrm{SF}_{6}$ mixture show no significant increase in COPR compared to a He/Ar mixture, only a positive shift of the COPR curve with cooling power of about 1.5 in $15 \mathrm{~W}$. [Work supported by Office of Naval Research, NASA, through the Pennsylvania Space Grant Consortium and the Applied Research Lab through the Enrichment and Foundation Program. The authors thank Scott Diehl for generous help with making these measurements.]

\section{1:00}

2aPAa9. Experimental verification of Rayleigh's interpretation for thermoacoustic Sondhauss-type oscillations. Guadalupe Huelsz and Eduardo Ramos (Centro de Investigación en Energía, UNAM, AP 34 CP 62580, Temixco, Mor. Mexico)

A century ago, Rayleigh [J. W. S. Rayleigh, The Theory of Sound (Dover, New York, 1945), Vol. 2, Sec. 322] gave a qualitative explanation of the acoustic power production in terms of the relative phase of the pressure wave and the heat transferred to the fluid. In quantitative terms his interpretation is equivalent to saying that a wave is promoted if the absolute value of this relative phase is less than $\pi / 2$, otherwise the wave is attenuated. The authors have demonstrated [G. Huelsz and E. Ramos, Int. Comm. Heat Mass Transfer 22, 71-80 (1995)] that in standing waves Rayleigh's criterion for acoustic power production can be translated to the relative phase of the pressure and the temperature oscillations in the limit when the distance to the solid boundary goes to zero; if the absolute value of this quantity is larger than $\pi / 2$ there is local acoustic power production. In the experimental work reported here, this criterion was used to provide 
experimental verification for Rayleigh's interpretation of the acoustic power production in a standing wave parallel to a wall with an axial temperature gradient. [Partial support for this work has been provided by DGAPA-UNAM IN103197 and CONACYT G0044E projects.]

\section{1:20}

2aPAa10. Thermoacoustic resonator and its sound source: Model of the coupling and experiments. Pierrick Lotton, Helene Bailliet, Michel Bruneau (Laboratoire d'Acoustique, IAM UMR 6613, Univ. du Maine, 72085 Le Mans Cedex 9, France, lotton@laum.univ-lemans.fr), and Vitali Gusev (Univ. du Maine, 72085 Le Mans Cedex 9, France)

Current thermoacoustic devices typically consist of a sound source loaded by both a "rear" cavity and the "front" thermoacoustic resonator. The main element placed in the resonator is a stack supporting a temperature gradient, sandwiched between heat exchangers. In thermoacoustic re- frigerators, heat transfer along the stack depends on the acoustic power flux available. In the framework of a previous model [Bailliet et al., "'Coupling between loudspeakers and thermoacoustic cavities,', submitted to Acta Acustica], this acoustic power can be determined knowing the electrical voltage applied to the source, an electrodynamic loudspeaker, the goal being to optimize the coupling between the loudspeaker and the thermoacoustic resonator. However, in this model, the temperature gradient along the stack is set to zero. The main purpose of this paper is to include in this model the temperature gradient along the stack. The expression of the input acoustic impedance of the thermoacoustic resonator is modified using an approximated analytical solution of the linear equation for the acoustic pressure inside the stack. The efficiency of the coupled system is expressed, yielding the highest acoustic power flux available in the stack (controlled in this study by LDA measurements) for a given electrical voltage applied to the loudspeaker.

TUESDAY MORNING, 16 MARCH 1999

ROOM MA042, 7:55 A.M. TO 12:20 P.M.

\title{
Session 2aPAb
}

\section{Physical Acoustics: Ultrasonic Imaging: Diagnostic Methods in Material Testing and Medicine}

\author{
Walter Arnold, Cochair \\ Fraunhofer Institute for Nondestructive Testing, University, Building 37, D-66123 Saarbrücken, Germany \\ Bernhard R. Tittmann, Cochair \\ Department of Engineering Science and Mechanics, Pennsylvania State University, University Park, \\ Pennsylvania 16802, USA
}

Chair's Introduction-7:55

\section{Invited Papers}

\section{8:00}

2aPAb1. Acoustic wave field imaging and measurement by scanning probe methods. Eduard Chilla (Paul-Drude Inst. for Solid State Electron., Nanoacoustics Group, Hausvogteiplatz 5-7, D-10117 Berlin, Germany, e.chilla@pdi-berlin.de)

Acoustic waves are widely utilized for the investigation of elastic properties of solids and layered systems. However, since the spatial resolution is limited due to Abbe's criteria, small structures can not be investigated by elastic waves using conventional techniques. These limitations can be overcome by applying scanning probe methods. A scanning acoustic force microscope (SAFM) and a scanning acoustic tunneling microscope (SATM) for the measurement of high-frequency surface acoustic waves were developed. The methods are based on the mixing at the nonlinearities of the tip-to-sample interactions. The amplitude and phase of the surface oscillation can be imaged with a lateral resolution in the nanometer range. The phase velocities of surface acoustic wave for propagation distances as small as $20 \mathrm{~nm}$ were measured. By using the SATM the surface oscillation was measured on the atomic scale. The SAFM was applied for the investigation of high-frequency interdigital transducers up to some GHz. Scattering and dispersion of elastic waves were studied. Recently, it has been shown that in-plane polarized surface acoustic waves can be detected by using a scanning acoustic probe technique. The phase velocity of Love waves in quartz-layered systems was determined.

$$
\text { 8:20 }
$$

2aPAb2. Acoustic images at the nanometer scale-Why and how? Andrew Kulik, Emmanuel Dupas, Frederic Oulevey, Delphine Gourdon, Nancy Burnham, and Gerard Gremaud (Ecole Polytechnique Federale de Lausanne, Phys. Dept., CH-1015 Lausanne, Switzerland, root@igahpse.epfl.ch)

For years, researchers have attempted super-resolution acoustical images. Using a subwavelength detector one can map acoustical fields with a spatial resolution determined by the size of the detector. A breakthrough approach is to use an atomic force microscope (AFM) in order to detect ultrasound. The lateral resolutions that have been achieved by various groups are better than $50 \mathrm{~nm}$. Different methods are in use to generate and detect the signal. Three main setups have been developed: (i) Contact-mode medium-frequency (typically $500 \mathrm{kHz}$ ) low-amplitude methods (SLAM), (ii) "mechanical diode" high-frequency (typically $5 \mathrm{MHz}$ ) high-amplitude pulsed setups (UFM), and (iii) contact-mode high-frequency (typically 5-400 MHz) methods using mixing of two ultrasonic waves through the nonlinearity of the tip-sample interaction. All three systems map different properties of the sample and have advantages and disadvantages. An overview of these methods will be illustrated by images and measurements. 
2aPAb3. Atomic force acoustic microscopy: Acoustical imaging on the $\mathbf{n m}$ scale. Ute Rabe and Sigrun Hirsekorn (Fraunhofer Inst. for Nondestructive Testing, Bldg. 37, Univ., D-66123 Saarbruecken, Germany, rabe@izfp.fhg.de)

High-resolution imaging techniques are important tools in materials evaluation and nondestructive testing. Conventional acoustical imaging techniques allow the determination of elastic properties and defect detection with a spatial resolution dependent on the wavelength of the imaging ultrasound. Research work over the last years has shown that this limit can be overcome by combining atomic force microscopy (AFM) with acoustic microscopy. Inducing ultrasonic vibrations in the contact formed by the sample surface with the sensor tip of an AFM opens its high lateral resolution to ultrasonic imaging. Different measurement techniques in which linear and nonlinear effects play a role are possible and have been demonstrated experimentally. An overview of the imaging techniques will be given. Theoretical models which allow the explanation of the image contrast and the derivation of quantitative surface qualities will be discussed.

\section{9:00}

2aPAb4. Material characterization using micro-machined air transducers. Butrus T. Khuri-Yakub, F. Levent Degertekin, Sean T. Hansen, and Neville R. Irani (Stanford Univ., E. L. Ginzton Lab., Stanford, CA 94305, khuri-ya@ee.stanford.edu)

This paper presents the application of micro-machined ultrasonic air transducers (MUT) to material characterization. The transducers are made of thin membrane capacitors with very thin vacuum gaps. A system analysis that details the design of the transducers and the transmitter and receiver electronics to deliver over a $100-\mathrm{dB}$ dynamic range while operating in the $1-5-\mathrm{MHz}$ range is presented. The transducers and electronics system are used both in pulse-echo and pitch-catch modes to evaluate materials. In transmission and reflection, the system is used to image defects in carbon epoxy composites. Transducers are designed, using Lamb wave theoretical calculations, to excite selected Lamb wave modes in composite and other materials. The transducers are then used to characterize the composites with respect to defect detection (via Lamb wave velocity changes) and material characterization (via slowness curve inversion).

9:20

2aPAb5. Imaging with a 16 000-element 2-D array. Ken Erikson and Tim White (Lockheed Martin, 2 Forbes Rd., Lexington, MA 02421)

Imaging with acoustical lenses in the low $\mathrm{MHz}$ frequency range using fully populated 2-D arrays offers the potential for high-resolution, real-time, 3D volume imaging, together with low power and low cost. A 2-D composite-piezoelectric receiver array bonded directly to a large custom-integrated circuit is the enabling technology for a new implementation of the original acoustical imaging paradigm. This $128 \times 128$ (16384 total) element transducer hybrid array (THA) uses massively parallel, on-chip signal processing and is intended for medical and underwater imaging applications. The system under development, which is the acoustical analog of a video camera, will be discussed in this paper.

9:40-10:00 Break

Contributed Papers

\section{0:00}

2aPAb6. Time-domain ultrasound diffraction tomography. $T$. Douglas Mast (Appl. Res. Lab., Penn State Univ., University Park, PA 16802, mast@sabine.acs.psu.edu)

A quantitative ultrasonic imaging method employing time-domain scattering data is presented. This method provides tomographic images of inhomogeneous media using scattering measurements made on a surface surrounding the medium of interest, e.g., on a circle for two-dimensional problems or on a sphere for three-dimensional problems. These scattering data are used to construct a time-domain analog of the far-field scattering operator. Images of compressibility variations are then reconstructed using a coherent combination of the far-field scattered waveforms, delayed and summed in a manner that numerically focuses on the unknown medium. This approach is closely related to synthetic aperture imaging; however, unlike conventional synthetic-aperture methods, the present method provides quantitative reconstructions of compressibility variations, analogous to frequency-compunded filtered backpropagation images weighted by the spectrum of the incident wave. Example reconstructions, obtained using synthetic data for two-dimensional scattering of wideband pulses, show that the time-domain reconstruction method can provide image quality superior to single-frequency reconstructions for objects of size and contrast relevant to medical imaging problems such as ultrasonic mammog- raphy. Reconstructions also illustrate the dependence of image quality on the number of incident-wave insonifications and on the range of scattering angles available for measurements.

10:20

2aPAb7. Transient elastography in biological tissues. Laurent Sandrin, Stefan Catheline, and Mathias Fink (ESPCI-LOA, 10 rue Vauquelin, 75005 Paris, France, laurent.sandrin@espci.fr)

Elastography is used in different ways to characterize soft tissues. Ophir uses static elastography to estimate strains in the tissue after a quasi-static compression. Strains can also be measured by sonoelasticity using mechanically forced low-frequency vibrations and the ultrasonic pulsed Doppler method (Parker and Sato). These techniques are subjected to bias due to unknown boundary conditions. In this article a technique called transient elastography is presented which is not sensible to boundary conditions. It uses a low-frequency pulsed vibration $(\sim 100 \mathrm{~Hz})$ and a cross-correlation technique to measure displacements on the order of $1 \mu \mathrm{m}$. This technique was first developed for one-dimensional measurements on the axis of the ultrasonic transducer. It is now used with an array of 64 transducers to get time-dependent two-dimensional displacements at a rate of 2000 frames per second. Movies of the shear wave propagation 
through homogeneous and inhomogeneous phantoms and biological tissues have been obtained. It will be discussed how to inverse near-field data in order to recover the medium shear viscosity and elasticity fields.

\section{0:40}

2aPAb8. Comparison between first and second harmonic imaging in ultrasound tomography. D. Kourtiche, A. Chitnalah, and M. Nadi (Lien, Université H. Poincaré, Nancy 1, BP 239, 54506, Vandoeuvre, France, kourtich@lien.u-nancy.fr)

In biomedical ultrasound imaging, quality of images can be enhanced using the second harmonic generated by nonlinear distorsion in the biological medium. Recent works have showed that the use of harmonics improves lateral resolution. The goal of this paper is to show the advantage of ultrasound tomography imaging using the second harmonic. The method of image reconstruction is based on diffraction Fourier theorem which relates the Fourier transform of the measured scattered field with the Fourier transform of the object function. This experimental setup is based on a $3-\mathrm{MHz}$ circular transducer $2 \mathrm{~cm}$ in diameter. The transmitted field is measured by a wide bandwidth hydrophone $1 \mathrm{~mm}$ in diameter. A cylindrical object is centered at $10 \mathrm{~cm}$ from the emitting transducer. A mechanical system permits the translation of the hydrophone step by step $(\lambda / 2)$. For each position, the signal is detected by a numerical oscilloscope and digitized. Data are transferred to the PC for posttreatment. The first and the second harmonics are then obtained from measurement by FFT. Tomographic imaging is obtained from the first and second harmonic measurements. These images are compared and the performance of the second harmonic imaging in terms of resolution is discussed.

\section{1:00}

2aPAb9. Amplitude dependence of down-converted acoustic waves due to a nonlinear force interaction. E. Dupas (Dept. of Phys., EPFL, CH-1015 Lausanne, Switzerland), G. Behme (Paul Drude Inst., Berlin, Germany), A. Kulik, N. A. Burnham (EPFL, CH-1015 Lausanne, Switzerland), E. Chilla, and H.-J. Froehlich (Paul Drude Inst., Berlin, Germany)

The interpretation of atomic force microscopy (AFM) data suffers from the lack of a detailed knowledge of the nonlinearity of the tip-sample force interaction. The AFM output signal as a function of the amplitude of two independent acoustic oscillations has been experimentally investigated in order to determine the interaction's power law. Two complementary experiments were performed: (1) Two separate $500-\mathrm{kHz}$ acoustic waves were propagated within the cantilever and through a cleaved mica sample; (2) 200-MHz surface acoustic waves (SAW) excited surface oscillations on a quartz sample in contact with the cantilever. In both experiments, the signals were amplitude modulated at a low frequency in order to record the dc mixing term. The cantilever vibration amplitudes at the modulation frequency, as well as at the difference frequency, were recorded using a lock-in amplifier. The experimental results have been compared with a physical model assuming a nonlinearity of the form $F(1 / d)^{n}$, with $F$ being the force, $d$ the penetration depth, and $n$ lying between 0 and 5. This model predicts an output signal depending approximately quadratically on each of the oscillation amplitudes. This approach to force interaction analysis will be evaluated.

\section{1:20}

2aPAb10. Double focus technique for quantitative measurements in time-resolved acoustic microscopy. Volker Haenel and Bernhard Kleffner (Fraunhofer Inst. of Biomed. Eng., Ultrasound Dept., Ensheimer Str. 48, D-66386 St. Ingbert, Germany)

Simultaneous measurement of sound velocity and thickness of thin specimens is a well-known technique in time-resolved acoustic microscopy. However, the applicability is restricted, because a substrate in contact with the back of the sample is required. Furthermore, the substrate must have an area without coating, because a reference signal from the substrate's surface is needed. In order to overcome these performance limitations, a double focus technique has been developed. By focusing the lens on the front, and afterwards on the back of the sample, only signals from the sample itself are required. Based on a simple geometrical model, the relevant acoustical equations for sound velocity and thickness were deduced analytically. Afterwards the method was applied to PVDF film in order to experimentally examine the feasibility of the approach. The measured results were compared with reference values and showed reasonable agreement. Based on these experiments, the influence of factors limiting the precision of the model, for example the spherical aberration, will be discussed, yielding an approach for an improved model. Eventually, the significance of the results for applying the method to manufacturing processes of thin films or foils, especially for online process control, will be described.

\section{1:40}

2aPAb11. A new method for nonlinear nondestructive testing of developing defects inside inhomogeneous solids. Vadim A. Robsman (Dept. of Information Technol., Transport Construction's Res. Inst., 1 Kolskay St., 129329 Moscow, Russia) and Oleg V. Rudenko (Moscow State Univ., 119899, Moscow, Russia)

Nonlinear acoustic interactions in defect solids subjected to the growing force load are studied both experimentally and theoretically. Three types of nonlinear processes are considered, which can be named as "wave-defect" interaction, "defect-defect" interaction (through the acoustic radiation), and the "defect-secondary radiation" one. Acoustic signals passed through the different building materials and constructions being under a load were recorded, as well as pulses radiated by growing defects at the increase in load. The nonlinear parameter of defect solid containing cracks was evaluated from the measured data. A new mathematical model describing the kinetics of statistical ensemble of cracks and based on nonlinear integrodifferential equation was developed. At an increase in force applied to the solid (including extremely high load up to a destructive one) new phenomena were observed, namely, jumps from one statistical distribution to another, radically different stages of nonlinear transformations in frequency spectra, and transitions from dynamic to stochastic behavior. Such processes can serve as new highly sensitive indicators of the defect solid structure and as a forerunner of catastrophic destruction.

\section{2:00}

2aPAb12. Investigations in ultrasonic tomography. Jean-Pierre Lefebvre, Philippe Lasaygues, Armand Wirgin, Sebastien Delamare (CNRS-Laboratoire de Mecanique et d'Acoustique, 31 chemin Joseph Aiguier, 13402 Marseille Cedex 20, France, lefebvre@lma.cnrs-mrs.fr), and Serge Mensah (Ecole Superieure de Mecanique de Marseille, IMT, 13451 Marseille Cedex 20, France)

Ultrasonic tomography (UT) is a technique to reconstruct the spatial distribution of some physical parameter of an object from measurements of the scattered field. The measurements are made for more or less dense sets of emitter and receiver positions and of frequencies of the interrogating wave. This inverse scattering problem was solved by using a Born approximation that leads to a particularly simple and attractive linear relation between the object function (OF) and the scattered field, particularly in the far-field (2-D or 3-D Fourier transform), making it possible, in principle, to reconstruct the $\mathrm{OF}$ in near real time for a sufficiently large set of scattering data. Ultrasonic tomography was investigated numerically and experimentally. Numerical simulations, using ideal measures with ideal objects, allow the examination in detail of the influence of various parameters such as the object's dimension and contrast, transducers bandwidth, etc. It allows one to analyze what happens when the Born approximation is no longer valid (high frequencies, high contrasts), to find limits of quantitative and qualitative imagery, and to imagine various improvement procedures (e.g., superresolution procedures leading to high resolution with low frequencies). Experimentations (with a mechanical and an antenna-based system) show the applicability of the method for medical and materials applications. 


\title{
Contributed Posters
}

Posters will be on display in the Poster Gallery from Monday to Wednesday, 15-17 March. Authors will be at their posters from 2:00 p.m. to 4:00 p.m. on Tuesday, 16 March.

2aPAb13. Nonlinear acoustic testing of defects for the evaluation of risk for insurance of thermal electric power stations. Vadim A. Robsman and Yury V. Novak (Dept. of Information Technol., Transport Construction's Res. Inst., 1 Kolskay St., 129329 Moscow, Russia)

The testing of defects in buidings, smoke tubes, groundworks of turbine generators, and other principal constructions of an electric power station without their removal from service was performed. Both externally exited acoustic pulses with narrow-band spectrum and acoustic emission pulses caused by vibration of power equipment were used as sources of structural information. Harmonics generation was observed at a small concentration of defects. Increase in concentration causes the growth of higher harmonic intensity and the broadening of their spectral lines. In highly damaged constructions, the spectral lines merge together into a continuous wideband spectrum. Acoustic emission can complicate the spectral distribution by introducing the random frequency components. It was discovered that the analysis of different stages of nonlinear evolution of spectra makes it possible to distinguish the different kinds of internal defects and, consequently, to predict the risk of emergency. This approach was put into use recently for insurance of thermal energy plants in Russia.l

2aPAb14. Experience in nonlinear wave testing of constructions inside the earthquake area. Vadim A. Robsman and V. E. Stepanyan (Dept. of Information Technol., Transport Construction's Res. Inst., 1 Kolskay St., 129329 Moscow, Russia)

In large-scale experiments on damaged engineering constructions disposed inside an earthquake area, the new nonlinear acoustic phenomena were observed which offer the possibility to evaluate the degree of dam- age and the strength of constructions. Specifically, the different parts of buildings (ground works, walls, etc.) were tested, as well as bridges damaged by catastrophic earthquake in Speatak, Armenia (1988). Such nonlinear technology was verified in the laboratory using a test seismic installation. Nonlinear testing was used later at the deep tunnel construction in rocks to predict the possible injury. Nonlinear effects were recorded in both transient internal radiation and in signals (regular and noise waves) generated by an external source. The direct relation was discovered between the rate of development of nonlinear distortion of spectra and the degree of damage of constructions subjected to earthquake shock.

2aPAb15. Experiments in solids using air-coupled ultrasound. D. A. Hutchins, D. R. Billson, and C. S. McIntyre (Univ. of Warwick, Coventry CV4 7AL, UK)

Electrostatic (capacitance) transducers have been designed and constructed for the imaging of solids in air, at frequencies of up to $1 \mathrm{MHz}$. The devices use a micromachined silicon backplate and a flexible membrane. The sensitivity and bandwidth of the resultant devices is such that signals can be propagated through solid samples in air. Examples will be given of imaging experiments in a wide range of materials, including fiber-reinforced polymer composites, paper, and metals. Measurements of material property can also be made, and examples will be given of how such measurements can be made at various temperatures. Particular reference will be made to measurements where it is possible to study changes in structure, by measurement of the elastic properties of the material.

\section{Session 2aPAc}

\section{Physical Acoustics: Interaction of Acoustic Fields with Suspended Particles I}

\author{
Robert E. Apfel, Cochair \\ Faculty of Engineering, Yale University, 9 Hillhouse Avenue, New Haven, Connecticut 06520-8286, USA \\ Ewald Benes, Cochair \\ Institut für Allgemeine Physik, Vienna University of Technology, A-1040 Vienna, Austria
}

Chair's Introduction-7:55

Invited Paper

\begin{abstract}
8:00
2aPAc1. Separation of aerosol fine particles by high-power ultrasound. Juan A. Gallego-Juarez, Iziar Gonzalez-Gomez, and Enrique Riera F. de Sarabia (Instituto de Acustica, CSIC, Serrano, 144, 28006 Madrid, Spain, jgallego@fresno.csic.es)

The application of high-intensity acoustic fields to an aerosol can produce the agglomeration of the suspended particles, shifting their size distribution to a large range. Acoustic agglomeration is a process in which acoustic forces cause particles to interact and eventually to collide. The complex mechanism of this process involves orthokinetic and hydrodynamic effects. The orthokinetic effect
\end{abstract}


refers to direct collisions produced among particles of different size and/or density which are differently entrained by the acoustic field. The hydrodynamic effects refer to particle interactions generated through the surrounding fluid by hydrodynamic forces induced by the acoustic field. One important hydrodynamic effect is the acoustic wake effect which is related to the asymmetry of the flow field around the moving particles. Acoustic agglomeration has a potential use in the separation of fine particles (smaller than $2.5 \mu \mathrm{m}$ ) which are emitted from industrial and residential combustion and from vehicle exhaust. In this paper recent theoretical and experimental advances in the knowledge of the basic mechanisms of acoustic agglomeration are presented, together with some results about the improvement of fine particle separation efficiency of conventional filters when the aerosol is previously treated with high-power ultrasound. [The authors acknowledge the support of CICYT-AMB96-A211-C02.]

\section{Contributed Papers}

8:20

2aPAc2. Studying drop cluster and array behavior using an acoustoelectric levitator. Yibing Zheng and Robert E. Apfel (Dept. of Mech. Eng., Yale Univ., New Haven, CT 06520-8286, yibing.zheng@yale.edu)

An acousto-electric levitator is developed for studying the formation and behavior of drop clusters and arrays. The device enables us to form suspended 2-D drop clusters and arrays by using electric and acoustic fields to levitate and manipulate drops. The spacing and the size of drops can be easily controlled by varying electric and/or acoustic field intensities. Experiments have been done on single- and multi-component drops, such as octane and dodecane, which are primary materials in natural fuels. Studies are focused on the evaporation rate of each drop due to the interaction in the array. The acoustic frequency has been increased from 28 $\mathrm{kHz}$ to more than $150 \mathrm{kHz}$ so that generation and control of smaller drops, associated with spray combustion, are possible. The formation process of clusters and arrays, and the force balance among drops are analyzed. A theoretical calculation based on the diffusion-controlled model is compared with these experimental results. This high-frequency acoustic levitator is also used to study solid particle clusters whose size is around $10 \mu \mathrm{m}$ by using a mixture of solid particles and liquid, and then letting the liquid evaporate. [Work supported by NASA through JPL Grant No. 958722 and NASA Grant No. NAG3-2147.]
8:40

2aPAc3. Generation of a standing wave field using crossing sound beams for acoustic micromanipulation. Teruyuki Kozuka, Toru Tuziuti, Hideto Mitome (Natl. Industrial Res. Inst. of Nagoya, AIST, 1-1 Hirate-cho, Kita-ku, Nagoya 462-8510, Japan, kozuka@nirin.go.jp), Toshio Fukuda, and Fumihito Arai (Nagoya Univ., Furo-cho, Chikusa-ku, Nagoya 464-8603, Japan)

A novel sound-field generation method for noncontact manipulation of particles has been studied experimentally. Although it was possible to trap particles in water at nodes of a standing wave field generated between a transducer and a reflector and to transport them using a frequency-shifting operation, there were several problems, such as unstable force due to resonance of the sound field and different movement of trapped particles. The present paper describes a method to generate a standing wave field using plural sound sources without a reflector. When two transducers were settled with their sound-beam axes crossing to each other, a standing wave field was generated in the crossing region. Changing the mutual phase of transmitting signals between the transducers, the sound field was shifted laterally, keeping the same form. When polystyrene particles were poured with a pipette into the sound field, they were trapped at the nodes of the sound-pressure distribution and were transported stably. Using three transducers whose sound-beam axes were crossed at an angle of $120^{\circ}$ to each other in a plane, a standing wave field was generated with a hexagonal node pattern, and two-dimensional manipulation of a particle was accomplished.

\section{Invited Papers}

9:00

2aPAc4. Ultrasonic micromanipulation of particles and effects of acoustic streaming. Hideto Mitome, Teruyuki Kozuka, and Toru Tuziuti (Natl. Industrial Res. Inst. of Nagoya, AIST, 1 Hirate-cho, Kita-ku, Nagoya 462-8510, Japan, mitome@ nirin.go.jp)

Noncontact manipulation of particles suspended in water has been studied using action of acoustic radiation pressure. A standing wave field in an unconfined vessel was used but the sound field was not necessarily resonant. When alumina suspension was poured into the sound field, particles were trapped and formed agglomeration. Changing the frequency continuously, the agglomerated particles were transported along the sound beam axis corresponding to the changes of wavelength. Selecting an appropriate frequency increment, they were transported in the opposite direction at different positions. Using a line-focused transducer with a back electrode divided into strips, it was possible to transport the particles perpendicular to the sound beam axis. A combination of these techniques realized two-dimensional manipulation without contact. Generation of acoustic streaming from the transducer to the reflector was observed even in the standing wave field due to nonuniformity of the sound field. To suppress this phenomenon for the stable manipulation, use of a tone-burst wave was proposed, noting the differences in the characteristic time between the streaming and trapping; the former is much longer than the latter and it is possible to keep the effective trapping force unchanged while suppressing the generation of streaming.

\section{9:20}

2aPAc5. Studies of particle separation using acoustophoresis. Todd L. Brooks and Robert E. Apfel (Dept. of Mech. Eng., Yale Univ., New Haven, CT 06520-8286, todd.brooks@yale.edu)

Particles in a liquid medium can be manipulated using the acoustic radiation force generated by a high-intensity ultrasonic standing wave. The resulting motion of the particles depends on the relative contrasts in particle density and compressibility with the surrounding host liquid. This allows the possibility of a separation technique that can distinguish particles based on these elastic properties, which is not possible with other separation methods. A flow-through resonator has been designed in which an acoustic standing wave is oriented perpendicular to the direction of fluid flow. This allows incoming particles to be moved perpendicular to the flow so that they can be separated into separate streams at the output end. Results will be shown for several different types of particles in a range of sizes $(5-100 \mu \mathrm{m})$. There is also a secondary force which can cause an attraction between particles. This force is usually considered negligible except when particles are very close together. Some preliminary calculations will be shown predicting the effects of the secondary particle force and these will be compared to observations. [Work supported by NASA through Grant No. NAG8-1351.] 
2aPAc6. Analysis of composite resonators for ultrasonic micromanipulation and separation. Martin Gröschl, Felix Trampler, Ewald Benes (Institut für Allgemeine Physik, Vienna Univ. of Technol., Wiedner Hauptstraße 8/134, A-1040 Wien, Austria, groeschl@iap.tuwien.ac.at), and Helmut Nowotny (Vienna Univ. of Technol., A-1040 Wien, Austria)

Piezoelectric composite resonators for the separation or manipulation of particles dispersed in a liquid by means of acoustic forces are investigated theoretically and experimentally. Such devices typically comprise at least four material layers: a piezoelectric transducer, a carrier (e.g., glass), the liquid (suspension), and an acoustic reflector. A mathematical matrix model for multilayer structures [M. Gröschl, Acust. Acta Acust. 84, 432-447 (1998)] is used to calculate the electric admittance spectrum of the resonator and results are compared to measurements obtained with a specialized computer-controlled measuring system. Additionally, the model allows the calculation of the spatial course of electric and acoustic field quantities (e.g., displacement amplitude, energy flow, energy density) along the multilayer structure. To characterize resonators for particle separation, the resonator performance number is introduced, which is a measure for the stored acoustic energy in the liquid (suspension) with respect to the total electric energy supplied by the driving source. It is shown that, for a given setup, the performance number depends strongly on frequency. The frequency range of maximum performance numbers defines the optimal operating range of the particle separator. [Work supported in part by the European Commission, Project \# FMRX-CT97-0156.]

\section{$10: 20$}

2aPAc7. Scattering enhancements and resonator frequency shifts resulting from the interaction between ultrasonic fields and suspended particles and bubbles. Philip L. Marston (Dept. of Phys., Washington State Univ., Pullman, WA 99164-2814), Christopher S. Kwiatkowski (Washington State Univ., Pullman, WA 99164-2814), and Harry J. Simpson (Naval Res. Lab., Washington, DC 20375-5350)

The radiation pressure of ultrasonic standing waves in water is known to cause suspended particles to migrate to energetically preferred locations. The spatial periodicity of the resulting bands of enhanced particle concentration makes possible significant acoustic signatures of this migration even if the volume fraction for the initially uniform spatial distribution is quite small. Our initial approach used acoustic Bragg scattering of a tilted high-frequency probe beam from the induced layers [H. J. Simpson and P. L. Marston, J. Acoust. Soc. Am. 98, 1731-1741 (1995); in Nonlinear Acoustics, edited by M. F. Hamilton and D. T. Blackstock (Academic, San Diego, 1998), Chap. 14]. The scattering is enhanced because the particles in the induced layers scatter sound with what amounts to the same phase. This effect was also observed with a probe wave collinear with the pump standing wave [C. S. Kwiatkowski, Ph.D. thesis, Washington State University, 1997]. The particle migration can also shift the frequency of the standing wave resonance [C. S. Kwiatkowski and P. L. Marston, J. Acoust. Soc. Am. 103, 3290-3300 (1998)] and a related frequency shift with dilute bubble clouds has also been observed. [Work supported by the Office of Naval Research.]

\section{0:40}

2aPAc8. Agglomeration and collection of suspended particles using resonant acoustic fields within porous media. Donald $\mathrm{L}$. Feke and Alan E. Penrod (Dept. of Chemical Eng., Case Western Reserve Univ., Cleveland, OH 44106-7217)

The collection of micron- to millimeter-size solids from flowing suspensions is of fundamental importance in many chemical and materials processing technologies. Recently, a technique has been developed that enables the collection of such solids within porous meshes and other filtration media having pore size up to 200 times larger than the particles being retained. This is accomplished by energizing the filtration medium with a low power, one-dimensional resonant acoustic field. In laboratory tests, collection efficiencies up to $90 \%$ have been achieved. The retention of the solids by the porous medium is reversible, and the solids can be easily flushed from the filter as soon as the acoustic field is terminated. In this presentation, data that illustrate the agglomeration and collection effects will be provided. The three possible mechanisms that can cause the observed phenomena will be discussed. In order to model the particle collection phenomena, a particle trajectory analysis has been completed. In this model, the path of individual particles toward elements of the porous mesh are calculated as a function of the fluid flow conditions and the acoustic force acting between the particle and collector. Design strategies for optimization of the particle collection phenomena will also be presented.

\section{1:00}

2aPAc9. Ultrasonic cell micro-manipulation and separation. W. T. Coakley (School of Biosciences, Cardiff Univ., Cardiff CF1 3TL, UK)

The manipulation of small ( $<5-\mu \mathrm{m}$-diam) eukaryotic cells and of bacteria (ca. 1.5- $\mu \mathrm{m}$ diameter) has been examined here in a number of batch and flow systems containing ultrasonic standing waves. The The different systems, involving plane and tubular ultrasonic transducers and a range of acoustic pathlengths, have been designed to treat suspension volumes of analytical scale, i.e., 5 $\mathrm{ml}$ to $50 \mu \mathrm{l}$ for both single-sample and "on-line" situations. The frequencies employed range from 1-12 MHz. Removal efficiencies of $99.5 \%$ have been achieved for yeast and for the bacterium Escherichia coli. The influence of secondary cell-cell interaction forces in determining the cell concentration dependence of harvesting efficiency in batch sedimentation systems is considered. The contributions of pulsing and of field modulation techniques to achieving high harvesting efficiencies are illustrated. Applications of standing wave radiation forces to (i) levitate cells in suspension for microscopic examination of cell interactions; (ii) to enhance particle agglutination immunoassay detection of cells or cellular products, and (iii) accelerate the aqueous biphasic separation of cells are presented. [Work supported by BBSRC and EU.] 


\title{
Session 2aPAd
}

\section{Physical Acoustics: Cavitation Physics and Sonoluminescence II}

\author{
Ronald A. Roy, Cochair \\ Department of Aerospace and Mechanical Engineering, Boston University, Boston, Massachusetts 02215, USA
}

\author{
Werner Lauterborn, Cochair \\ Drittes Physikalisches Institut, Universität Göttingen, Bürgerstrasse 42-44, 37073 Göttingen, Germany
}

\section{Invited Papers}

\section{8:00}

2aPAd1. Stimulating sonoluminescence. Joachim Holzfuss and Matthias Rüggeberg (Inst. für Angew. Physik, TU Darmstadt, Schlossgartenstr. 7, 64289 Darmstadt, Germany)

Single-bubble sonoluminescence is characterized by the light emission of an acoustically driven bubble in a liquid. It is due to an enormous compression of the bubble volume, where a change in volume by six orders of magnitude is followed by a violent collapse. The question has been asked if the violence of the collapse can still be increased. In the theory of nonlinear dynamical systems, methods have been developed to control systems toward higher energetic response. As a complex system that is excited within a resonant cell is being dealt with, harmonic series expansions of the driving signal have been used. It is shown that already two-mode signals with optimized amplitudes and phases are able to boost sonoluminescence. The difficulties concerning multimode signals are analyzed experimentally and numerically. The effects of nonsinusoidal excitation concerning spatial and temporal bubble dynamics are presented. [Work supported by the DFG (SFB 185).]

8:20

2aPAd2. Sonoluminescence light emission. Sascha Hilgenfeldt (DEAS, Harvard Univ., 29 Oxford St., Cambridge, MA 02138, sascha@stokes.harvard.edu), Detlef Lohse (Univ. of Twente, The Netherlands), and Siegfried Grossmann (Univ. of Marburg, Marburg, Germany)

Extending the hydrodynamical/chemical approach to single-bubble sonoluminescence (SBSL) to include light emission, it is shown that a few well-known, simple physical concepts are sufficient to account for the observed characteristics of emitted light pulses: Rayleigh-Plesset bubble dynamics, polytropic gas heating, dissociation of molecular gases, and thermal radiation of optically thin bodies. With these ingredients, a model can be developed which yields correct widths, shapes, intensities, and spectra for the pulses of emitted SBSL light. These quantities all depend in a characteristic way on each other, as well as on experimental parameters like driving pressure, water temperature, or dissolved gas concentration. A direct comparison of the theoretical results to experimental findings shows good agreement. The theory furthermore predicts that strongly forced xenon bubbles should show a dependence of the pulse width on light wavelength, in contrast to argon bubbles. Moreover, if upscaled SBSL bubbles of larger size at larger driving pressures can be created (e.g., using smaller driving frequencies), the resulting light emission intensities should be greatly increased.

8:40

2aPAd3. Computed optical emissions from a star in a jar. William Moss (Lawrence Livermore Natl. Lab., L-200, 7000 East Ave., Livermore, CA 94550, wmoss@1lnl.gov)

A sonoluminescing bubble has been modeled as a thermally conducting, partially ionized plasma. The model accounts for most of the observed experimental trends, including (i) the asymmetric pulse shape; (ii) the temperature and driving pressure dependence of the pulse width and intensity; and (iii) spectral shapes, in particular, the 300-nm peak in the spectrum of xenon sonoluminescence. The agreement between the calculations and the data, as well as the models predictions of almost every experimental trend, leave little doubt that the spectral and temporal properties of the emissions of a single sonoluminescing bubble are due to adiabatic- or shockinitiated thermal emission from a cool dense plasma. [Work was performed under the auspices of the U. S. Department of Energy by Lawrence Livermore National Laboratory under Contract No. W-7405-Eng-48.]

9:00

2aPAd4. Water vapor and sonoluminescence. Andrew J. Szeri and Brian D. Storey (Univ. of California, Dept. of Mech. Eng., Berkeley, CA 94720-1740, aszeri@me.berkeley.edu)

In recent work, it has been shown that segregation of gas mixtures within sonoluminescence bubbles is driven by strong thermal and pressure gradients inside the bubble [B. D. Storey and A. J. Szeri, "Mixture segregation within sonoluminescence bubbles," in review]. Although these rather exotic types of diffusive transport are often neglected, they are of crucial importance in a number of applications. These effects conspire to increase the fraction of the lighter gas in a mixture of noble gases near the center, and to increase the fraction of the heavier gas near the bubble wall at the moment of extreme collapse. This results in a greater energy concentration at the bubble center. While the temperature and pressure peaks have a duration (FWHM) of only a few hundred 
picoseconds at most, it has been found that appreciable mixture segregation persists for several orders of magnitude longer. Armed with this new understanding of how gas mixtures behave, it is possible to investigate more deeply the question of water vapor within the bubble. Several investigators have shown that the presence of water vapor has important consequences for compression versus shock heating, for hydrodynamics of the bubble collapse, and for chemistry.

\section{Contributed Papers}

\section{9:20}

2aPAd5. Optimal resonant stimulation of sonoluminescence. Matthias Rüggeberg and Joachim Holzfuss (Inst. fuer Angew. Physik, TU Darmstadt, Schlossgartenstr. 7, 64289 Darmstadt, Germany)

In single-bubble sonoluminescence, a bubble is levitated and forced to oscillate by a standing sound wave. Once per cycle, at the maximum compression of the bubble volume, the emission of a pulse of visible light is observed; due to the strong bubble collapse, a shock wave is emitted in the liquid also. It was found that these shock waves can be visualized by the means of a schlieren method. Using a stroboscopic illumination of the bubble, its dynamical behavior and the shocks can be recorded in slow motion by a video camera. Experimentally measured velocities of the shock waves were compared with numerical calculations. Further, the recorded shock waves contain information about the bubble position and collapse time. In the regime of instable sonoluminescence, sudden changes in bubble position depending on bubble size were observed. The phenomenon in the context of interactions of the bubble with the complex sound field is discussed. [Work supported by the DFG (SFB 185).]

9:40-10:00 Break

10:00

2aPAd6. Sonoluminescing bubbles rectify argon: Comparing predictions with latest experiments. Sascha Hilgenfeldt (Div. of Eng. and Appl. Sci., Harvard Univ., 29 Oxford St., Cambridge, MA 02138) and Detlef Lohse (Univ. of Twente, 7500 AE Enschede, The Netherlands, lohse@tn.utwente.nl)

For the ASA meeting in Hawaii in 1996, the Session Chair, Robert Apfel, posed a challenge to the theoreticians working on single-bubble sonoluminescence (SBSL): experiments should be suggested and predictions made. The crucial predictions following from the hydrodynamical/ chemical approach to SBSL were phase diagrams for SBSL bubbles, the rectification of argon in SBSL air bubbles, the existence of stable nitrogen bubbles, and the ejection of chemical reaction products from the SBSL bubble [S. Hilgenfeldt, D. Lohse, and M. Brenner, Phys. Fluids 8, 2808 (1996); D. Lohse et al., Phys. Rev. Lett. 78, 1359 (1997); D. Lohse and S. Hilgenfeldt, J. Chem. Phys. 107, 6986 (1997)]. Meanwhile, many beautiful experiments have been done by Crum and Matula, by Apfel and Ketterling, by Gaitan and Holt, by Suslick et al., by Barber, Putterman et al., and by many others. In this talk a comparison between these experiments and above predictions is presented.

\section{0:20}

2aPAd7. Sonoluminescence and acoustics within nonlinearly driven cavities. Ralf Guenther (Naturwissenschaftliches und Medizinisches Institut (NMI), D-72770 Reutlingen, Germany, guenther@nmi.de)

The field distributions inside a nonlinearly driven cavitation bubble are obtained as a function of the dynamics of the bubble radius. They represent a straightforward generalization of standing wave solutions within a one-dimensional cavity to nonlinearly driven cavities. By coupling the solution to the Rayleigh-Plesset equation, a bubble equation is obtained which includes heat transfer from the bubble to the liquid and the spatial variation of the pressure field. The range of validity of the perturbational solution is dependent on the nature of the bubble content, and is largest for monoatomic gases. The inclusion of the internal field distributions into the simulation of the bubble dynamics is possible with very small computational effort. Applications to sonoluminescence are discussed. [Work supported by the German Ministry of Education and Research.]

\section{0:40}

2aPAd8. The search for electrical discharges from single- and multibubble sonoluminescence. Vassilious J. Bezzerides, Thomas J. Matula, and Lawrence A. Crum (Appl. Phys. Lab., Univ. of Washington, 1013 NE 40th St., Seattle, WA 98105, matula@apl.washington.edu)

Previous research supported the notion that electrical discharges resulted from the collapse of bubbles in cavitation fields [G. Giminez and F. Goby, in Cavitation and Inhomogeneities in Underwater Acoustics, edited by W. Lauterborn (Springer-Verlag, New York, 1980), pp. 101-107]. Further research was performed in a cavitation field, as well as with a single cavitating bubble. The cavitation field was generated in a standing wavefield using gassy water, resulting in a small confined region of cavitation. Single-bubble cavitation was also performed in this system, but with degassed water. Two methods were used to detect electrical pulses. In one case a small probe consisting of two fine wires coupled to a low-current differential amplifier was placed near the cavitation bubble(s). Signals were only observed when the probe was in contact with the cavitation field (no signals were observed in the single-bubble system). The second method utilized a coiled loop of wire surrounding the levitation cell, connected to a high-bandwidth lock-in amplifier. In this case, the reference frequency was scanned from about $25 \mathrm{kHz}$ to more than $20 \mathrm{MHz}$. Signals were observed at harmonics of the drive frequency. [This research supported by DOE-EM.]

\section{1:00}

2aPAd9. Multibubble sonoluminescence at 2 MHz: Optical spectral data and attempt at interpretation. T. Lepoint, E. Hankenne, F. Lepoint-Mullie, and N. Vogelet (Lab. de Sonochimie et d'Etude de la Cavitation, Institut Meurice, Av. Emile Gryzon 1, B-1070 Brussels, Belgium)

In order to obtain information on the physical conditions created by transient cavitation bubbles imploding at acoustic frequencies (fac) of therapeutic interest, the UV-visible spectral distribution of multibubble sonoluminescence at $1.7 \mathrm{MHz}$ is focused on. Original optical spectra (wavelength range: 220-950; resolution: $0.24 \mathrm{~nm}$ ) will be reported for aqueous solutions containing various alkali-metals chlorides and dissolved noble gases. Special emphasis will be put on the profile of $D 1$ and $D 2$ lines associated with the reduced metals [i.e., (i) their blending toward the red zone (much less than for fac $20 \mathrm{kHz}$ ); (ii) the absence of measurable shift and broadening; (iii) the absence of fusion of the $D$ lines]. Several scenarios for (i) the reduction of the cation and (ii) the surrounding of the emitting metal atoms will be discussed. [The present authors-particularly T.L. and F. L-M.- are very grateful to Dr. J-L. Domanchain and G. Nivez (Jobin-Yvon I.S.A.).] 
2aPAd10. The stability of microbubbles and sonoluminescence. U. H. Augsdörfer, ${ }^{\text {a) }}$ A. K. Evans, and D. P. Oxley (The Sci. and Eng. Res. Ctr., De Montfort Univ., Leicester LE1 7BH, England)

Sonoluminescence (SL), the light emission of a micron-sized bubble of gas trapped in water by an acoustic field, is associated with the collapse of a bubble nonlinearly oscillating under the sound field. Recent advances have made it possible to trap a single bubble at the pressure antinode of a standing wave, where it collapses and reexpands with the periodicity of the applied sound and emits light with every cycle. A critical feature of SL is the spherical symmetry of the bubble. If the collapse is violent, irregularities in the spherical shape of the bubble may develop towards the end of the collapse. As a result, the bubble may get destroyed shortly after the collapse. Due to the microscopic size of the bubble, molecular fluctuations constitute an additional force to which the gas-liquid interface is exposed. This additional force causes irregularities in the spherical shape of the bubble. The stability of a bubble is discussed by analyzing results obtained from hydrodynamic calculations of the bubble dynamics and the fluid dynamical processes outside the bubble taking molecular fluctuations into consideration. Results of these calculations reveal a surprising stability behavior and are in good agreement with observed behavior in singlebubble SL experiments. ${ }^{\text {a) }}$ ua@dmu.ac.uk
2aPAd11. Numerical simulations of stationary single-bubble sonoluminescence. Vladimir N. Nogin, Nikolay G. Karlykhanov, Gennadiy V. Kovalenko, and Vadim A. Simonenko (Russian Federal Nuclear Ctr., ITP, P.O. Box 245, 456770 Snezhinsk, Chelyabinsk region, Russia,sva@sva.ch70.chel.su)

In one-dimensional approximation, the approach was developed to simulate the regime of stationary behavior (groves, collapse, and rebouncing) for a gas-filled single bubble located in the center of a spherical water-filled vessel under an external resonant periodic sound field. The simulations were performed for realistic equations of state for water and gas (air or argon), with identification of the shock wave in the bubble, with account of surface tension on the water-gas interface, and heat conduction for the matter in the bubble (molecular and radiative). The bubble radius dependence versus time was obtained for varying external conditions: amplitude and frequency of acoustic field, temperature of water, and magnitude of surface tension. Calculated parameters for a stationary bubble (pressure in bubble vicinity, minimal and stationary radii of bubble) were demonstrated to show a good agreement with experimental data. [Work supported by CRDF.]

\title{
Session 2aPPa
}

\section{Psychological and Physiological Acoustics: The Role of Peripheral Compression in Hearing}

\author{
Mary Florentine, Cochair \\ Department of Speech, Language Pathology and Audiology, Northeastern University, 1000 Forsythe Street, \\ Boston, Massachusetts 02115, USA \\ Brian C. J. Moore, Cochair \\ Department of Experimental Psychology, University of Cambridge, Downing Street, Cambridge CB2 3EB, UK
}

Chair's Introduction-7:55

Invited Papers

8:00

2aPPa1. Amplification and compression in the cochlea. Ian Russell (School of Biological Sci., Univ. of Sussex, Falmer, Brighton BN1 9QG, UK, I.J.Russell@ sussex.ac.uk)

The large dynamic range of the auditory system is due to processes within the cochlea which amplify low-level and compress high-level signals. Both the amplification and compression are associated with outer hair cell function and are reflected in their voltage responses to tones. Outer hair cells control the mechanical properties of the cochlear partition for frequencies close to the peak of the traveling wave which propagates along it. Thus they also control the mechanical input to the inner hair cells and excitation of the auditory nerve fibres. The amplification of basilar membrane responses can exceed $60 \mathrm{~dB}$ for high-frequency, low-level tones close to the characteristic frequency of the measurement place. For responses to levels above about $40 \mathrm{~dB}$ SPL, hair cell and basilar membrane responses are compressive and increase at a rate close to $0.2 \mathrm{~dB}$ per dB SPL. The cochlear efferent system has been implicated in controlling compression, but indications of the underlying mechanism of compression can be gained from measurements of the spatial distribution of basilar membrane responses both along its length and width, and from temporal changes in these responses to tones.

\section{8:40}

2aPPa2. Measurements of peripheral compression using forward masking and pulsation threshold. Christopher J. Plack (Dept. of Psych., Univ. of Essex, Wivenhoe Park, Colchester CO4 3SQ, UK) and Andrew J. Oxenham (Dept. of Speech-Lang. Pathol. and Audiol., Northeastern Univ., Boston, MA 02115)

Development of a reliable psychophysical measure of the basilar-membrane (BM) response function is of great importance, not least as a diagnostic tool for sensorineural hearing loss. Described here are attempts to estimate the response function using forward masking and pulsation threshold. Both techniques measure, in effect, the masker level needed to mask a higher frequency signal as a 
function of signal level. In the forward-masking design, the signal is a 4-ms tone burst presented $2 \mathrm{~ms}$ afer a 100 -ms masker. In the pulsation-threshold design, 200-ms signal bursts are alternated with 200-ms masker bursts and the listener determines the masker level at which the signal just begins to sound "continuous" as opposed to "pulsed." This is assumed to occur when the masker and signal excitation levels are equal at the BM place tuned to the signal. The experiemnts are based on the finding that the BM response function is roughly linear for stimuli with frequencies well below characteristic frequency (CF). A plot of masker level at threshold against signal level provides, therefore, an estimate of the BM response to the (CF) signal. Results from normal and impaired listeners will be presented and the relative merits of the techniques discussed.

\section{9:00}

2aPPa3. Nonlinear cochlear processing of harmonic complexes. Marjorie R. Leek (Army Audiol. and Speech Ctr., Walter Reed Army Med. Ctr., Washington, DC 20307, leek@wrair-emh1.army.mil)

Some harmonic complexes with component phases that change monotonically with harmonic frequency are characterized by temporal waveforms with relatively flat envelopes. With an appropriate choice of component phases, the fine structure within periods represents an upward or downward sweep of instantaneous frequency. One such class of phase selections creates "Schroeder-phase waveforms," named after the developer of the phase algorithm. These stimuli have been used recently in a number of psychoacoustic studies because of some intriguing characteristics of the waveforms, along with some compelling hypotheses regarding the interactions of the waveforms with cochlear processing mechanisms. Properties of Schroeder waveforms will be discussed in relation to hypotheses regarding internal alteration of waveform shapes by normal and impaired cochlear processing. Studies will be reviewed that involve the effects of phase selection on discrimination of temporal waveforms, as well as masking by Schroeder-phase stimuli in normal and impaired auditory systems. Results from these studies appear to reflect properties of the traveling wave within the cochlea, as well as evidence of active nonlinear gain in normal cochleas. [Work supported by NIH DC00626.]

\section{9:20}

2aPPa4. Loudness and ABR findings with short signals: More compression than expected? Birger Kollmeier, Jesko Verhey, Oliver Wegner, and Torsten Dau (AG Medizinische Physik, Universität Oldenburg, Germany)

Previous work on loudness perception was primarily focused on stationary stimuli. However, knowledge about the processing and perception of temporally varying sounds is of special interest for various applications, such as, e.g., speech perception and hearing aids. We therefore studied the loudness of brief sounds both with psychophysical and electrophysiological methods (i.e., auditory evoked potentials) as a function of the stimulus bandwidth. The loudness summation effect appeared to be larger for brief stimuli than for long stimuli. Also, the magnitude of wave $\mathrm{V}$ in ABR recordings for brief stimuli with equal subjective loudness was found to depend on the stimulus bandwidth. Both results indicate that the effective compression for short signals is larger than for long signals. This finding is in conflict with predictions derived with current loudness models or with a model of the effective processing in the auditory system. The consequences of these findings for the nonlinear compression stage in auditory models will be discussed.

9:40

2aPPa5. On the relation between peripheral compression and the growth of loudness. Sbren Buus (Commun. and Digital Signal Processing Ctr., ECE Dept. (409 DA), Northeastern Univ., 110 Forsyth St., Boston, MA 02115), Mary Florentine (Northeastern Univ., Boston, MA 02115), Torben Poulsen (Tech. Univ. Denmark, DK 2800 Lyngby, Denmark), and Hannes Müsch (Northeastern Univ., Boston, MA 02115)

This presentation reviews similarities and differences between normal listeners' pure-tone loudness functions and the compressive basilar-membrane, BM, input-output function. Loudness matches between pure tones and multitone complexes with widely separated components reveal that the loudness function can be modeled by a modified power function with local exponents around unity near threshold and 0.2 at moderate levels [S. Buus et al., J. Acoust. Soc. Am. 104, 399-410 (1998)]. Loudness matches between short and long tones show that the SPL difference at equal loudness is a nonmonotonic function of SPL and is largest at moderate levels [S. Buus et al., J. Acoust. Soc. Am. 101, 669-680 (1997)]. These data can be explained by a loudness function that is flattest at moderate levels, where its local exponent also is around 0.2 . This value is close to that obtained for BM input-output functions. The steepening of the loudness function at low levels is qualitatively similar to BM measurements. The steepening at high levels is absent in BM data when the state of the animals' cochleae is carefully controlled. One possible explanation is that the cochleae of normal adult listeners are in worse condition than those of carefully treated animals. [Work supported by NIH/NIDCD.]

\section{0:00}

2aPPa6. Relationship between loudness perception and peripheral compression in subjects with unilateral cochlear hearing loss. Brian C. J. Moore, Deborah A. Vickers (Dept. of Exp. Psych., Univ. of Cambridge, Cambridge CB2 3EB, England), Christopher J. Plack (Univ. of Sussex, Brighton BN1 9QG, England), and Andrew J. Oxenham (Northeastern Univ., Boston, MA 02115)

Loss of compression on the basilar membrane (BM) associated with outer hair cell damage $(\mathrm{OHC})$ is thought to be the main factor underlying loudness recruitment in people with cochlear heating loss. To assess this idea, subjects with unilateral cochlear hearing loss were tested. Recruitment was measured by obtaining loudness matches between tones presented alternately to the two ears. Compression was estimated from the slopes of growth-of-masking functions in forward masking. The ratio of slopes for a masker centered well below the signal frequency and a masker centered at the signal frequency gives a measure of BM compression at the place corresponding to the signal frequency [A. J. Oxenham and C. J. Plack, J. Acoust. Soc. Am. 101, 3666-3675 (1997)]. The measures of loudness recruitment were used to derive a parameter $\mathrm{HL}_{\mathrm{OHC}}$, the amount of the hearing loss attributable to OHC damage [B. C. J. Moore and B. R. Glasberg, Auditory Neurosci. 3, 289-311 (1997)]. The value of $\mathrm{HL}_{\mathrm{OHC}}$ is directly related to the amount of recruitment. The estimated compression decreased systematically as $\mathrm{HL}_{\mathrm{OHC}}$ increased from 0 to $55 \mathrm{~dB}$. These results are consistent with the idea that loudness recruitment is directly related to loss of compression on the BM. [Work supported by the MRC, UK.] 


\section{Contributed Papers}

\section{0:40}

2aPPa7. Nonlinear amplification in the cochlea-Implications of a computer model. Martin Steurer (Dept. of Otorhinolaryngology, Univ. of Vienna, Waehringer Guertel 18-20, A-1090 Vienna, Austria)

In the cochlear amplifier the output is fed back to the input. Namely, the contraction of the outer hair cells (OHCs) is believed to be controlled by the deflection of the ciliary bundle caused by the movements of the tectorial membrane. The contraction of the OHCs leads to an intensified movement of the basilar and tectorial membrane, and by this closing the loop. Such a model implies minimal response time of up to $300 \mathrm{~ms}$ to reach the steady state of amplification and even more time to return from maximum amplification to zero movement (except thermal movement). These time limits can be reduced when accepting intensity-dependent optimal frequencies of the cochlea amplifiers. The optimum frequency defined by best phase shift at a certain place of the basilar membrane starts nearby the characteristic frequency of the basilar membrane and increases with increasing intensity. Therefore, the peak amplification for a fixed frequency moves in the apical direction with increasing intensity. The results of this model simulated on a computer show improved time responses and explain how to control phase shifts for the optimum frequency in the cochlear amplifier.

\section{1:00}

2aPPa8. Monaural, cross-frequency coincidence detection as a mechanism for decoding perceptual cues provided by the cochlear amplifier. Michael G. Heinz (Speech and Hearing Sci. Prog., MIT, Cambridge, MA 02139 and Hearing Res. Ctr., Boston Univ., Boston, MA 02215, mgheinz@mit.edu), Laurel H. Carney, and H. Steven Colburn (Boston Univ., Boston, MA 02215)

Basilar membrane (BM) response properties associated with the cochlear amplifier are consistent with broadened BM tuning as level increases from approximately 20 to $90 \mathrm{~dB}$ SPL or higher. Associated with broadened tuning are compressive BM magnitude responses and leveldependent phase changes. To evaluate the perceptual significance of the cochlear amplifier, the associated nonlinear response properties of the auditory nerve (AN) and physiologically realistic mechanisms for decoding the perceptual cues they provide were studied. Most AN fibers' rate and synchrony responses to tones are saturated above $40 \mathrm{~dB}$ SPL; however, nonlinear phase changes with level are encoded in AN responses up to 90 $\mathrm{dB}$ SPL or higher. Broadened tuning produces an increase in the correlation between adjacent AN fibers. Monaural coincidence detection is a physiologically realistic mechanism that can extract nonlinear phase cues across AN fibers with similar characteristic frequencies over a wide dynamic range. Methods from statistical decision theory [Colburn, J. Acoust. Soc. Am. 54, 1458-1470 (1973)] were combined with an analytical AN model to provide a quantitative framework for evaluating the potential of a coincidence detector to explain human performance in pure-tone intensity and frequency discrimination. [Work supported by NIH Grant No. T32DC00038 and NSF Grant No. 9601215.]

\section{1:20}

2aPPa9. Benefits of nonlinear active gain for detection of brief probes in Schroeder-phase complex maskers. Van Summers and Philip Seitz (Army Audiol. and Speech Ctr., Walter Reed Army Med. Ctr., Washington, DC 20307-5001, vsummers@erols.com)

Masking period patterns for positive and negative Schroeder-phase complexes were determined for tonal probes at 1 and $4 \mathrm{kHz}$. Maskers included components from $200-5000 \mathrm{~Hz}$ of a $100-\mathrm{Hz}$ fundamental. The 5 -ms probes had onsets occurring 153,155.5, 158, 160.5, or $163 \mathrm{~ms}$ fol- lowing masker onset. For listeners with normal hearing, thresholds in the positive Schroeder-phase masker varied by as much as $25 \mathrm{~dB}$ depending on the position of the probe within the masker period. Thresholds in the negative Schroeder-phase maskers were nearly constant as probe onset varied. The maskers were more nearly equal in effectiveness for listeners with sensorineural hearing loss and in testing at high presentation levels. The findings support an interpretation involving differences in the shape of the basilar-membrane waveform generated by each masker, and influences of nonlinear active gain on these internal responses. The positive Schroeder-phase masker appears to produce a highly modulated basilar membrane response. Nonlinear active gain enhances the signal-to-masker ratio for signals presented during low-amplitude "troughs" of these modulated internal waveforms. Consistent with this account of the present findings, active gain is reduced or eliminated at high presentation levels and with cochlear damage.

\section{1:40}

2aPPa10. Loudness fluctuation and temporal masking in normal and hearing-impaired listeners. Josef Chalupper (Inst. of Man-Machine-Commun., Tech. Univ. München, Arcisstr. 21, D-80333 München, Germany, cha@mmk.e-technik.tu-muenchen.de)

The model of fluctuation strength as proposed by Fastl is based on the level difference $\Delta L$ between the maximum and minimum of the temporal masking pattern of dynamically varying sounds. Compared to normalhearing subjects this level difference can be strongly reduced in hearingimpaired listeners. Recent findings, however, suggest that perceived fluctuations in hearing-impaired listeners are magnified by recruitment. Based on fitted loudness fluctuations-measured by a categorical scaling procedure-for each subject a "uniform loud noise" was generated, having equal loudness across all critical bands. These noises were amplitude modulated with different rates and depths. In experiment 1 , subjects had to judge "how strong (not how fast) fluctuates the loudness" by marking one of seven verbal categories. In experiment 2 , the $\Delta L$ 's of the various noises were measured by determining the thresholds in the envelope's maximum and minimum. Although measured level differences were markedly reduced in the hearing impaired, the perceived loudness fluctuations are nearly the same for normal and hearing-impaired listeners. These results support the idea that the model of fluctuation strength can be applied to hearing-impaired listeners by transforming the difference in level into the corresponding individual difference in loudness. [Work supported by Geers.]

\section{2:00}

2aPPa11. Relation of long-term adaptation to the loudness-function slope. William Hellman (Dept. of Phys. and Hearing Res. Ctr., Boston Univ., 590 Commonwealth Ave., Boston, MA 02215, hellman@buphyc.bu.edu) and Rhona Hellman (Northeastern Univ., Boston, MA 02115)

The decline in loudness over time, defined as simple loudness adaptation, is modeled by introducing temporal factors into the nonadapted loudness function. This modification generates effective time-dependent intensity and threshold shifts. Model predictions for several frequencies are in accord with recent psychophysical measures [Hellman et al., J. Acoust. Soc. Am. 101, 2176-2185 (1997)]. In contrast to the time constant description of adaptation, the present method produces a direct connection to properties of the loudness function. A consequence of this connection is the demonstration that the increase in loudness adaptation with decreases in SL is linearly related to the loudness-function slope in $\log -\log$ coordinates. Moreover, the good agreement between the measured and predicted loudness-adaptation data implies a consistency and stability in the use of numbers for loudness judgments. 


\title{
Session 2aPPb
}

\section{Psychological and Physiological Acoustics: Spatial Factors in Binaural Hearing (Poster Session)}

\author{
D. Wesley Grantham, Chair \\ Bill Wilkerson Center for Otolaryngology and Communication Science, Department of Speech and Hearing Science, \\ Vanderbilt University Medical Center, Nashville, Tennessee 37212, USA
}

\section{Contributed Papers}

Posters will be on display in the Poster Gallery from Monday to Wednesday, 15-17 March. Authors will be at their posters from 10:00 a.m. to 12:00 noon on Tuesday, 16 March.

2aPPb1. Near-field localization in echoic rooms. Scott Santarelli, Norbert Kopco, Barbara G. Shinn-Cunningham (Dept. of Cognit. and Neural Systems, Boston Univ., 677 Beacon St., Boston, MA 02215, shinn@cns.bu.edu), and Douglas Brungart (Armstrong Lab., WrightPatterson AFB, OH 45433-7901)

The current study examines the ability of subjects to indicate the distance and direction of sources within $1 \mathrm{~m}$ of the head in a medium-sized, echoic classroom. Two conditions were tested in the same group of subjects. In the first, subjects were seated in the center of the room, relatively far from any hard reflective surfaces. In the second condition, subjects were located at the same position in the room, but a $12 \times 4$ enamelcovered wallboard was positioned next to the listener to create an additional artificial wall approximately 6 in. from the left ear of the listeners. The initial hypothesis was that previous localization results from tests in anechoic space would be nearly indistinguishable from the results in the first condition, since for sources near the head, the direct-to-reverberant energy ratio in this first condition would be very large. However, it was believed that the addition of a single, short-latency echo might bias some localization judgments, particularly judgments of distance and elevation. Instead the results indicate that localization accuracy and variability are comparable for the two echoic conditions, but that both measures of localization ability are worse in echoic conditions than in anechoic conditions.

2aPPb2. The effect of sentence onset asynchrony on call sign detection and message intelligibility in a simulated "cocktail party." Brian D. Simpson (Dept. of Psych., Wright State Univ., 3640 Colonel Glenn Hwy., Dayton, OH 45435, bsimpson@sdl.psych.wright.edu), Robert S. Bolia (Veridian, Dayton, OH 45431), Mark A. Ericson, and Richard L. McKinley (Air Force Res. Lab., Wright-Patterson Air Force Base, OH 45433-7901)

Previous research has demonstrated that the spatial separation of multiple simultaneous talkers improves detection and intelligibility of a critical speech signal among non-signal speech events [Nelson et al., J. Acoust. Soc. Am. 103, 2341-2342(A) (1998); M. L. Hawley et al., J. Acoust. Soc. Am. 99, 2596(A) (1996)]. However, few findings on the effects of varying phrase onsets have been reported [J. C. Webster and P. O. Thompson, J. Acoust. Soc. Am. 26, 396-402 (1954)]. The purpose of the present study was to investigate the effect of varying the interval between sentence onsets on call sign detection and message intelligibility. The relative onset times of two to eight temporally overlapping phrases were varied systematically in both spatially separated and nonspatially separated conditions on the horizontal plane. The phrases were presented virtually to five normal-hearing listeners. All possible temporal positions of the target phrase were examined. Results will be discussed in the context of listening to speech in real-world situations. [Work supported by AFOSR.]
2aPPb3. Speech intelligibility in real and virtual adverse auditory environments. Nancy L. Vause (U.S. Army Res. Lab., Human Res. and Eng. Directorate, Aberdeen Proving Ground, MD 21005) and D. Wesley Grantham (Vanderbilt Univ. Med. Ctr., Nashville, TN 37212)

The purpose of this investigation was to examine the effects of real and virtual adverse conditions on speech intelligibility. The experiment was designed to examine the effect of presentation method on speech intelligibility in conditions of noise and reverberation as signal-to-noise ratio was systematically varied. Additionally, this investigation evaluated the influence of talker gender on speech intelligibility in anechoic and reverberant environments as a function of signal-to-noise ratio and presentation method. Speech intelligibility scores were obtained from 21 normal hearing subjects using a nonsense syllable test. The syllables were recorded in three environments (diotic anechoic, virtual anechoic, and virtual reverberant) with three signal-to-noise ratios $(0,5,9 \mathrm{~dB})$ using two simultaneous masking sources. The findings indicate that (a) traditional diotic presentation of these stimuli degrades speech intelligibility compared to virtual presentation and may not accurately represent real-world performance; (b) the signal-to-noise ratios did not influence performance trends between presentation modes, as intelligibility improved with increasing SNR; (c) reverberation decreased intelligibility although the effects were not as dramatic as expected; and (d) using these stimuli, the female talker tokens were more easily identified than male talkers in a virtual presentation, but not generally for diotic presentation. [Work supported by NICDC and U.S. Army.]

2aPPb4. Monaural and binaural minimum audible angles for virtual sound sources. Robert S. Bolia (Veridian, 5200 Springfield St., Dayton, OH 45431, rbolia@falcon.al.wpafb.af.mil), Alan D. Musicant, and Daniel A. Gajewski (Middle Tennessee State Univ., Murfreesboro, TN 37132)

A number of researchers have employed the minimum audible angle (MAA) paradigm to investigate the precision with which listeners can discriminate differences of sound-source location in the free field [A. W. Mills, J. Acoust. Soc. Am. 30, 237-246 (1958); W. M. Hartmann and B. Rakerd, ibid. 85, 2031-2041 (1989)]. Surprisingly, this has been done only for binaural listening conditions, although Man's capacity for monaural localization is well documented [J. R. Angell and W. Fite, Psych. Rev. 8, 225-246 (1901); R. A. Butler, R. A. Humanski, and A. D. Musicant, Perception 19, 241-256 (1990)]. In the present study, monaural and binaural MAAs were determined using a broadband stimulus for seven virtual sound-source positions in the horizontal plane. Listeners indicated via a three-alternative, forced-choice paradigm whether the stimulus presented in the second interval appeared to move clockwise, counterclockwise, or not at all, with respect to that presented in the first interval. 
Results will be discussed in terms of data obtained in previous studies of binaural MAA, and compared with data on monaural and binaural localization acuity.

2aPPb5. The cocktail party effect with conversational speech. James W. Kondash, Robert H. Gilkey, Scott K. Isabelle, and Brian D. Simpson (Dept. of Psych., Wright State Univ., Dayton, OH 45435)

Most often the cocktail party effect has been evaluated for relatively simple stimuli (e.g., isolated words or simple sentences masked by noise) in anechoic environments. Here, a stimulus situation is used which is similar to that employed by Plomp [Acustica 34, 200-211 (1976)] (i.e., continuous discourse for both target and masker) in a reverberant room. However, instead of asking subjects to make a subjective judgment of intelligibility, the "tracking procedure" of DeFillipo and Scott is used [J. Acoust. Soc. Am. 63, 1186-1192 (1978)] to simulate a conversation. This procedure measures the number of words per minute (WPM) successfully transmitted between the target talker and the subject. Preliminary results show increases in WPM of about $20 \%$ when the masker is separated from the target by $90 \mathrm{deg}$ in the horizontal plane relative to the case when both the target and masker are at the same location in front of the subject. (The change in S/N necessary to maintain constant WPM between these conditions indicates that the magnitude of the effect is similar to that observed by Plomp.) Separating the target and masker(s) in elevation has little effect on WPM. [Work supported by AFOSR and the Ohio Board of Regents.]

2aPPb6. Monaural azimuth sound localization with linguistic and nonlinguistic stimuli. Barbara S. Muller and Pierre Bovet (Dept. of Psych., Univ. of Geneva, 9 rte de Drize, 1227 Geneva, Switzerland, Barbara.Muller@pse.unige.ch)

Many studies have been conducted to measure monaural azimuth sound localization performances with different sounds varying in frequency and complexity, but few are using language. This experimental design included phonologically and semantically different sentences, that is, they either contained fricative consonants or not, and all sentences were presented right way out or reversed. Analysis of response correctness of 30 subjects showed that localization performance is better with the left ear than with the right ear, but that neither the phonological nor the semantic aspect of the stimuli have any influence on localization accuracy. Analysis of response times of the subjects revealed an effect of the stimuli's nature. Reversed sentences as well as those containing fricative consonants need more time to be localized than other stimuli. These results suggest that monaural sound localization is independent of phonological or semantic attributes of the signal, but that linguistic stimuli are processed more quickly than nonlinguistic ones.

2aPPb7. Aided and unaided performance on a clinical test of sound localization. Joan Besing, Janet Koehnke (U.S. Army Res. Lab, Human Res. and Eng. Directorate, AMSRL-HR-SD, Bldg. 520, Aberdeen Proving Ground, MD 21005, jkoehnke@arl.mil), Patrick M. Zurek, Kosuke Kawakyu (Sensimetrics Corp.), and Jennifer Lister (Univ. of South Alabama)

A test of sound field localization using two small loudspeakers has been developed to evaluate performance on this task in typical clinical settings. The intensity-difference stereophony technique, incorporating interspeaker intensity differences, is used to create phantom sound images in the horizontal plane at locations between the actual loudspeakers. When the intensity of the stimulus from the speakers is the same, the source is perceived straight ahead; as the interspeaker intensity difference increases, the perceived source location moves toward the speaker with the more intense stimulus. Using speakers located at \pm 40 degrees, localization of short phrases has been measured for subjects with normal hearing and subjects with hearing loss. The subjects with hearing loss were tested with and without their hearing aids. Results indicate that subjects with normal hearing have good localization with errors averaging 8.0 degrees. Unaided, subjects with hearing loss generally have larger localization errors than subjects with normal hearing, although there is a wide range of per- formance. With their hearing aids, the majority of subjects with hearing loss have poorer localization than without their hearing aids. Repeated administration of this test over a period of days or weeks indicates good test-retest reliability. [Work supported by NIH, NIDCD/SBIR No. DC02435.]

2aPPb8. Auditory spatial attention influences discrimination of interaural time differences: Evidence from a probe-signal paradigm. Andrew J. Sach, Nicholas I. Hill, and Peter J. Bailey (Dept. of Psych., Univ. of York, York YO10 5DD, UK, a.sach@psych.york.ac.uk)

Performance on a same-different ITD discrimination task was used as a metric for auditory spatial attention. In several variants of a probe-signal procedure, attention was directed as detailed below. In all experiments, performance was better for signals lateralized on the expected side of the head. In experiment 1 , a lateralized auditory cue preceded the signal. Lest this functioned not simply as an attentional cue but also as a spatial landmark, experiment 2 used no cue but manipulated expectation by presenting trials more frequently on a particular side. To establish whether the effects in experiment 2 generalized to all sounds within the spatial focus or were attributable simply to greater familiarity with the expected stimulus, a rove was incorporated in the pitch (experiment 3 ) or the timbre (experiment 4 ) of the signals on each trial. Experiment 5 introduced trial-by-trial visual cueing which, necessarily endogenous, provided evidence for topdown attentional control. Further work established whether analogous effects are seen on a frequency discrimination task, and when sounds are lateralized by IID. [Work supported by UK BBSRC.]

2aPPb9. Effects of low-frequency thresholds on sound-source identification in the frontal horizontal plane using interaural time difference cues. Stuart Gatehouse, Catherine Lever, Christian Lorenzi, and Patrick Howell (MRC Inst. of Hearing Res., Royal Infirmary, Glasgow G31 2ER, Scotland)

Eight listeners with symmetric sensorineural hearing loss were investigated using a sound-source identification paradigm, to study localization abilities in noise for sources in the frontal horizontal plane. Stimulae were low-pass filtered click trains making available the primary cue of interaural time differences. Compared to performance of a normally hearing reference group, the listeners with sensorineural hearing loss exhibited systematic deficits in localization ability which were strongly related to hearing level. However, parallel detection experiments also showed deficits and the localization data might be a function of simple audibility. The listeners were further tested using a number of binaural hearing aid fittings for which detectability was returned to levels displayed by the normalhearing listeners. Aided localization ability did improve but still showed residual deficits compared to the normal-hearing references and, furthermore, still had a systematic relationship with hearing level. The gradient of this relationship was approximately half that in the unaided data. The results suggest that sensorineural hearing loss compromises the use of interaural time difference cues to achieve sound-source identification and that the deficit is in part independent of audibility considerations.

2aPPb10. Acoustic weapons? Sources and propagation of strong sound. Jürgen Altmann (Experimentelle Physik III, Universität Dortmund/Institut für Experimentalphysik III, Ruhr-Universität Bochum, 44780 Bochum, Germany, Altmann@EP3.Ruhr-Uni-Bochum.DE)

Acoustic weapons are under research and development in a few countries. Reliable information is scarce; allegations, mainly in the military press, have stressed the infrasound region. By means of a literature review and own analysis potential strong sources from infra- to ultrasound were identified. Speakers with horns, sirens, and whistles can produce acoustic powers of kilowatts to tens of kilowatts. Explosive blasts have no upper limit. Repetitive explosions, e.g., of a fuel-air mixture, might produce powers on the order of megawatts. Most sources would be too large to be 
handheld. Propagation of strong sound to some distance meets several problems, however. Diffraction impedes or prevents directed propagation, in particular at low frequencies. Sound of high intensity propagates nonlinearly, deforming into shocked waves with strong attenuation. The level at a given distance depends in a complicated way on details, such as source level, source size, frequency, wavefront form, and humidity. Keeping certain sound-level limits, e.g., at the ears of targeted people, would require very complex processing and automatic control. Acoustic weapons may turn out cumbersome and may not add important new options for the military; this could facilitate preventive limits up to a ban.

\title{
Session 2aSAa
}

\section{Structural Acoustics and Vibration: Inverse Problems in Structural Acoustics II}

\author{
Bjorn A. Petersson, Cochair \\ Department of Aeronautical and Automotive Engineering, Loughborough University of Technology, \\ Loughborough, Leicestershire LE11 3TU, UK \\ Sean F. Wu, Cochair \\ Department of Mechanical Engineering, Wayne State University, Detroit, Michigan 48202, USA \\ Chair's Introduction-7:55
}

\section{Contributed Papers}

\begin{abstract}
8:00
2aSAa1. Parameter estimation of layered structures with one accessible surface. G. Dixon, J. L. Horner, and B. A. Petersson (Dept. of A.A.E.T.S., Loughborough Univ., Ashby Rd., Loughborough, Leicestershire LE11 3TU, UK, g.dixon@lboro.ac.uk)

It is frequently desirable, for inspection purposes, to test a layered structure nonintrusively. In practice, it is not always possible, or convenient, to access both surfaces. The aim of this paper is to determine the accuracy with which the parameters of a layered structure can be found, measurements being taken from only one surface. Using a matrix propagator model, a layer over a half-space is examined. Modeling point excitation, the sensitivity of the response to changes in the parameters of the layers are presented. The information gained will support an extended study, focusing upon systems with many layers, some of which may be composed of inhomogeneous materials.
\end{abstract}

\section{8:20}

2aSAa2. Order tracking using the Vold-Kalman tracking filter. Svend Gade, Henrik Herlufsen, and Hans Konstantin-Hansen (Brüel \& Kjær, Skodsborgvej 307, DK-2850 Nærum, Denmark, sgade@bk.dk)

The Vold-Kalman filter allows for the high-performance simultaneous tracking of orders in systems with multiple independent axles. The interactions associated with close and crossing orders give rise to beating and complex psychoacoustic phenomena. With this new filter and using multiple tachometer references, waveforms as well as amplitude and phase may be extracted without the beating interactions. The Vold-Kalman filter provides several filter shapes for optimum resolution and stopband suppression. Orders extracted as waveforms have no phase bias, and may hence be used in synthesis and tailoring. The input to Vold-Kalman filtering is time history data. The class of algorithms based on Kalman filters shows no data between preselected orders, but has fine resolution, and is independent of slew rates. The calculation speed may be an issue if a large number of orders is to be extracted. This paper shows an example of the various steps of order tracking using the Vold-Kalman method applied on a simple single-shaft motor. Filter shapes are discussed and an example of decoupling of orders from a multishaft system is demonstrated. The VoldKalman filter is implemented in PULSE, the Brüel \& Kjær Multianalysis System.

\section{8:40}

2aSAa3. Characterization of complex systems from inverse radiative transfer measurements. John Burkhardt (Dept. of Mech. Eng., U.S. Naval Acad., Annapolis, MD 21402)

A technique is proposed for the characterization of complex systems using measurements of time-dependent backscattered acoustic intensity. Utilizing a statistical model of system disorder, radiative transfer theory is used to model backscattered acoustic intensity. An algorithm for the estimation of system characteristics from observed backscatter is developed and presented. The technique is tested by numerically simulating the backscattered acoustic intensity from a disordered acoustic half-space with prescribed characteristics. Inversely estimated system characteristics are then compared with known system parameters.

\section{9:00}

2aSAa4. Indirect determination of input forces generated by the pump of a watercraft under operating conditions: Some guidelines. J-M. Janin, Y. Champoux, and N. Attala (G.A.U.S., Dept. of Mech. Eng., Univ. of Sherbrooke, Sherbrooke, QC J1K 2R1, Canada, Jean-Marc.Janin@gaus.gme.usherb.ca)

Engineers are currently able to calculate the dynamic characteristics of a complex structure using numerical tools. However, from an optimization point of view, it would be more valuable to predict correctly the actual response of the structure under working conditions. That requires that the engineers have the ability to determine precisely the input forces that are injected to the structure while operating. The direct measurement of the input forces is often impossible. That is due to the difficulties in mounting 
transducers and to the additional modifications it implies on the structure. Indirect measurement of forces has already been applied to a few industrial structures with some success, but no guidelines on the method have been given yet. In this paper, the work that has been done on the determination of the forces generated by the pump of a watercraft under real operating conditions, i.e., in the water, where direct measurements are made with difficulty, is presented. A comparison of the different inversion techniques (pseudoinversion, TLS) has been made. Attention here has been focused on clearly defining limitations and guidelines concerning the use of indirect force measurement methods in order to obtain results with a good confidence level.

\section{9:20}

2aSAa5. Sensor proximity error in the conformal near-field acoustic holography and its solution by using the nonsingular boundary integral equation. Jeong-Guon Ih and Sung-Chon Kang (Ctr. for Noise and Vib. Control, Dept. of Mech. Eng., Korea Adv. Inst. of Sci. \& Technol., Taejon 305-701, Korea, ihih@sorak.kaist.ac.kr)

In the conformal near-field acoustic holography (NAH) using the boundary element method (BEM), the transfer matrix relating the vibroacoustic properties of source and field depends solely on the geometrical condition of the problem. This kind of NAH is known to be very powerful in dealing with the sources having irregular boundaries. When the vibroacoustic source field is reconstructed by using this conformal NAH, one tends to position the sensors as close as possible to the source surface in order to get rich information on the nonpropagating wave components. The conventional acoustic BEM based on the Kirchhoff-Helmholtz integral equation has the singularity problem in the very-near field of the source surface: computation error increases very rapidly as approaching from the far field. This problem originated from the singular kernel of the fundamental solution of the BIE and can influence the reconstruction accuracy. In this paper, the holographic BIE is reformulated to remove the singularity by introducing an additional propagating plane wave. Reconstructed results by the conventional and the nonsingular BIE are compared for a simple radiator model. It is observed that the nonsingular formulation can yield accurate vibro-acoustic transfer matrix and thus improve the resolution of the reconstructed source field.

9:40

2aSAa6. A vibroacoustic inverse problem regularized by SVD technique coupled with Miller's method. C. Marquis-Favre (LASH/ DGCB URA CNRS 1652, ENTPE, rue M. Audin, 69518 Vaulx-en-Velin, France, Cathy.Marquis@entpe.fr), N. Hamzaoui, and C. Boisson (LVA, INSA, 69621 Villeurbanne, France)

This communication presents the resolution of a vibroacoustic inverse problem. This latter is a typical ill-posed problem introduced by a Fredholm integral equation of the first kind. This formulation is used in the mathematical model established for an approach dealing with numerical construction of the vibroacoustic transfer function for sound radiation prediction from an industrial structure operating in its real environment [Marquis et al., ICA (1995)]. The construction of the vibroacoustic transfer function is based on the use of acoustic pressures and vibratory velocity measurements defining the structure in situ. The acoustic pressures are data for computation of a density function allocated to points sources distributed on the structure. This calculation lies in solving a vibroacoustic inverse problem. This is carried out with the SVD technique. The regularization process is based on the Miller's method using a priori information on the solution and on the measurement errors contaminating the data. Some numerical simulations for an academic vibroacoustic system illustrate the sensitivity of different parameters and their influence on the application conditions of the approach. Some practical indications concerning SVD and the regularization are given and some results show the regularization effects and the consequences in the presence of measurements errors

TUESDAY MORNING, 16 MARCH 1999

ROOM EB301, 10:00 A.M. TO 12:20 P.M.

\title{
Session 2aSAb \\ International Workshop on Active Noise and Vibration Control
}

\section{Structural Acoustics and Vibration: Active Control of Structural Vibration}

\author{
M. Osman Tokhi, Cochair \\ Department of Automatic Control and Systems Engineering, University of Sheffield, Sheffield S1 3JD, UK \\ Andy Von Flotow, Cochair \\ Hood Technology Corporation, 1750 Country Club Road, Hood River, Oregon 97031, USA
}

\section{Invited Papers}

10:00

\begin{abstract}
2aSAb1. Nonlinear modeling of the forward path in a mechanical active control system. Linus Pettersson, Lars Hakansson, Ingvar Claesson, and Thomas Lago (Dept. of Appl. Signal Processing, ITS/HK-R, 37225 Ronneby, Sweden, LINUSPETTERSSON@ITS.HK-r.se)
\end{abstract}

In most active control applications the estimate of the forward path is closely related to the performance of the controlled system. Nonlinearities in the forward path are most likely to degrade the performance. In the active control of machine tool vibration in a lathe, the plant under control principally consists of two physical parts: amplifier and a tool holder construction with integrated actuators. One applicable actuator type was used and it was based on highly magnetostrictive material. However, these types of actuators generally have nonlinear behavior. To obtain deeper understanding on the dynamic properties of the plant under control and thereby obtain a solid base for the feedback control of the machine tool vibration, nonlinear modeling of the forward path was proposed. Two methods were used: The approach of neural networks and the reverse multiple-input single-output by Bandat have been used in order to describe the forward path as accurately as possible. In the active control system of machine tool vibration in a lathe the forward path is the transfer from the actuator amplifier to the output from the accelerometer mounted on the toolholder shank in the toolholder construction. 
2aSAb2. Control of vibration of flexible manipulators. M. O. Tokhi (Dept. of Automatic Control and Systems Eng., The Univ. of Sheffield, Mappin St., Sheffield S1 3JD, UK, o.tokhi@ sheffield.ac.uk)

Flexible manipulator systems are receiving increasing attention due to their advantages over conventional rigid manipulators. The advantages of flexible manipulators are faster response, lower energy consumption, relatively smaller actuators, higher payload to weight ratio and, in general, less overall cost. However, due to their flexible nature, induced vibrations appear in the system during and after a positioning motion. This implies that in developing a control strategy for such systems, both the rigid body as well as flexible motion (vibration) have to be taken into account. A considerable amount of research work has already been carried out on the vibration control of flexible manipulators. These range from open-loop control to closed-loop control strategies of fixed and adaptive nature. This paper presents an investigation into the development of several approaches for vibration control of flexible manipulators. A single-link flexible manipulator is considered. Open-loop control techniques, including Gaussian-shaped and filtered command methods, and closed-loop control approaches of fixed and adaptive nature, including joint-based collocated control and hybrid collocated and non-collocated control, are developed. These are verified within simulation and practical rig-based environments. Finally, a comparative assessment of the performance of these strategies in vibration suppression of flexible manipulators is provided.

\section{Contributed Papers}

10:40

2aSAb3. Real-time control of a vibrating plate excited by a turbulent boundary layer. Emmanuel Friot, Marc Georgelin (CNRS-LMA, 31 Chemin Joseph Aiguier, 13402 Marseille Cedex 09, France, friot@1ma.cnrs-mrs.fr), Christophe Durand, and Gilles Robert (LMFA, 69131 Ecully, France)

Optimal feedback control was designed and successfully implemented to reduce the vibrations of a steel plate excited by a turbulent boundary layer. One side of the plate was equipped with piezoelectric sensors and actuators. The other side was imbedded in the wall of the CNRS-LMFA anechoic wind tunnel. Measurements of the wall pressure at flow speed ranging from 70 to $130 \mathrm{~m} / \mathrm{s}$ showed that the plate was excited by a standard boundary layer; the parameters of a Corcos model for the wall pressure were identified from flow data. For control a state-space model for the plate was first identified from the actuator-to-sensor transfer functions; nine plate modes in the $0-1000-\mathrm{Hz}$ range were taken into account. Covariance matrices for the boundary layer excitation were also identified from vibration data. An optimal linear quadratic Gaussian (LQG) controller was then designed from the plate and noise model. The LQG algorithm was implemented on the CNRS-LMA multichannel control system and tested at three flow velocities. A $10-\mathrm{dB}$ reduction of the vibration level was achieved for the three modes mostly excited by the boundary layer.

\section{1:00}

2aSAb4. Semiactive damping of vibration in mixing or propelling systems. George Vachtsevanos and Peng Wang (School of Elec. and Computer Eng., Georgia Inst. of Technol., Atlanta, GA 30332-0250, gjv@ee.gatech.edu)

Vibration damping for mixing or propelling systems is investigated in this research, through the use of semiactive control techniques. The mixing or propelling systems considered consist of a motor mounted on some support, a long shaft driven by the motor, and a propeller. Such sytems can be found in many engineering areas like chemical and materials engineering. Because the shaft is long and the propeller is working in liquids, occurrence of vibrations in such a system is inevitable. Damping of the vibration is critical to prolong the life of the motor and thus of the whole system. However, it is difficult to establish an accurate mathematical model for the operation of the mixing or propelling systems since they involve a great deal of material characteristics and structure dynamics. This paper proposes a dynamic wavelet neural network to model the relationship between vibration observations and operating parameters. This model is them employed to design a semiactive controller regulating the operating variables so that vibrations can be damped. Experimental and simulation results are presented. The experimental setup consists of an AC motor, a triaxial transducer, a data acquisition module, in addition to LABVIEW software from a Dual-400-MHz Pentium-II workstation.
$11: 20$

2aSAb5. A distributed active vibration absorber for sound radiation and vibration control. C. R. Fuller and P. E. Cambou (Vib. and Acoust. Labs., Mech. Eng. Dept., Virginia Tech, Blacksburg, VA 24061)

This paper discusses the analytical and experimental development of a distributed active vibration absorber (DAVA) designed to reduce sound radiation and vibration over extended areas of elastic structures. The distributed vibration absorber, in contrast to the classical vibration absorber, consists of a continuous active elastic layer covered with a continuous mass distribution. The distribution of the mass layer can be optimally designed to reduce either sound radiation or vibration over selected areas or frequency bands for particular structures. The paper first discusses an analytical model of the DAVA based upon variational techniques and used in conjunction with a genetic algorithm to design the mass distribution optimally. Simulated results on vibration and sound radiation control for a vibrating beam are presented and are compared to experimental realizations of the DAVA. The analytical and experimental tests confirm that the DAVA can passively attenuate vibration and sound radiation through an extended area and over an extended frequency range. The DAVA is shown to outperform the classical point vibration absorber significantly. The introduction of an active signal to the DAVA is seen to provide significant additional sound and vibration reduction. The DAVA is demonstrated to provide effective passive and active vibration and sound control in a conformal, compact, skin treatment. [Work funded by the ONR, Dr. Kam Ng, Technical Monitor.]

\section{1:40}

2aSAb6. Multichannel feedback control for the isolation of equipment from base vibration. Marc J. Serrand and Stephen J. Elliott (ISVR, Univ. of Southampton, Highfield, Southampton SO17 1BJ, UK, msc97ms@isvr.soton.ac.uk)

Passive systems for the isolation of equipment from base vibration involve an inherent compromise between good high-frequency isolation, which requires low values of isolator damping, and limited excitation of the rigid body modes, which requires high values of isolator damping. It is known that a single-channel active control system using "skyhook damping," in which a secondary force acts in proportion to the absolute velocity of the equipment, can give good damping in a lumped mass-springdamper system without compromising the high-frequency isolation. In this experimental study a multichannel skyhook damper was used to study the isolation of a rigid mass from the vibrations of a flexible base plate. With the actuators and sensors wired together and the system excited symmetrically, a single-channel controller was initially implemented which not only showed reductions in the equipment vibration of up to $40 \mathrm{~dB}$ at the heave resonance at $15 \mathrm{~Hz}$, but also showed substantial reductions at modes of the flexible base plate below about $200 \mathrm{~Hz}$. The multichannel controller was also able to control the pitching mode of the equipment which is apparent when the system is not excited symmetrically. 
the active force. The structures of the linear controller, employing both the feed-back "sky-hook"-type additional damping and the feed-forward vibration control for both types of electro-pneumatic transducers, will be illustrated. Both systems, of different control structure, are capable of mitigation of the vibration exposure of the driver by 8 to $10 \mathrm{~dB}$, measured by a standardized method. The theoretical possibility of use of active or semi-active vibration control systems for smart/intelligent vibration and shock control will be briefly discussed. The idea is to use already available active or semi-active means to rapidly modify the damping properties of a linear oscillatory system according to commands based on fast evaluation of input vibration and so intelligently adapt the structure to the character of vibration excitation.

TUESDAY MORNING, 16 MARCH 1999

ROOM H1028, 7:55 A.M. TO 12:20 P.M.

\title{
Session 2aSCa
}

\section{Speech Communication: Data Based Speech Synthesis II}

\author{
Nick Campbell, Cochair \\ Advanced Telecommunication Research Laboratory, Hikari-dai 2-2, Seika-cho, Kyoto 619-02, Japan \\ Juergen Schroeter, Cochair \\ AT\&T Laboratories-Research, 180 Park Avenue, Florham Park, New Jersey 07932-0971, USA
}

Chair's Introduction-7:55

Invited Papers

8:00

2aSCa1. Rules, measures, and corpora for speech synthesis. Yoshinori Sagiksaka (ATR-ITL, Kyoto, Japan)

A speech synthesis system is characterized by three factors: control rules, evaluation measures for rule development, and speech corpora to quantify and test these rules. In traditional speech synthesis by rule, the latter two factors have not been clearly acknowledged as system building constituents. Unclear acknowledgment of these two factors has often made it difficult for researchers to develop and evaluate a system consistently with objective measures. At ATR, corpus-based speech synthesis was proposed more than a decade ago to cope with this deficiency. In this talk, research efforts in corpus-based synthesis are overviewed to show how these three factors have been studied. Through this introduction, it is stressed that both clear formulation of engineering problems and basic scientific investigation of human mechanism are quite important to build a system efficiently and to understand human characteristics properly in a scientific fashion. To figure out the future problems, research efforts combining subjective evaluation and objective measures will also be introduced on the control of temporal characteristics for speech synthesis.

8:20

2aSCa2. An overview of Microsoft's Whistler text-to-speech system. X. D. Huang (Microsoft Research, Redmond, WA 98052)

The data-driven approach can significantly facilitate the process of creating text-to-speech (TTS) systems for a new language, a new voice, or a new style. As such, Whistler TTS engine was designed to benefit from automatically constructed model parameters. Efforts to improve Whistler with the use of additional training data and better learning algorithms that make full use of these data will be reviewed. Training data have been augmented for a number of speakers. To better use these data, the hidden Markov model speech recognition system has been used to segment the training corpora and select more representative acoustic units. The classification and regression tree was used for both grapheme to phoneme conversation and unseen triphone generalization. Speech signal reconstruction was based on the mixed excitation source-filter model that leads to better compression of the acoustic inventory. A number of ways to smooth the spectral parameters were also studied to minimize the concatenation distortion. To improve automatically extracted prosodic templates, the learning process was refined with an analysis-by-synthesis approach. However, the coverage remains a challenge for the data-driven approach to make Whistler produce synthetic speech that resembles the original speaker. This is especially true for the prosody model.

8:40

2aSCa3. Data-driven speech synthesis. Nick Campbell (ATR-ITL, Kyoto, 619-02 Japan)

Described in this paper are the theoretical background and implementation of a speech synthesis engine that uses an index of features describing a natural speech source to provide pointers to waveform segments that can then be re-sequenced to form novel utterances. By efficiently labelling the features in speech that are minimally sufficient to describe the perceptually relevant variation 
in acoustic and prosodic characterisitcs, reduce the task of synthesis to "retrieval" rather than "replication," and is reduced reuse of original waveform segments is possible without the need for (perceptually damaging) signal processing. The drawback of this system is that it requires a large corpus of natural speech from one speaker, but current improvements in data-storage devices and cpu technology have overcome this problem. The style of the corpus speech determines the style of the synthesis, but experiments with corpora of emotional speech confirm that by switching source corpora one can easily control the speaking style. By shifting "knowledge" out of the synthesizer into the source data an engine is produced that can work on any adequately labelled speech corpus. The interesting work for the future lies in determining which features are relevant to capture the variation in speech, and how they can be best described.

\section{9:00-9:20 Discussion}

\section{9:20}

2aSCa4. The AT\&T Next-Gen TTS System. Mark C. Beutnagel, Alistair D. Conkie, Juergen Schroeter, Yannis Stylianou, and Ann K. Syrdal (AT\&T Labs—Res., 180 Park Ave., Florham Park, NJ 07932-0791, jsh@ research.att.com)

The new AT\&T TTS system for general U.S. English text is based on best-choice components picked from the AT\&T Flextalk TTS, the Festival System from the University of Edinburgh, and ATR's CHATR system. From Flextalk, it employs text normalization, letter-to-sound, and (optionally) baseline prosody generation. Festival provides general software-engineering infrastructure (modularity) for easy experimentation and competitive evaluation of different algorithms or modules. Finally, CHATR's unit selection was modified to guarantee the intelligibility of a good $n$-phone ( $n=2$ would be diphone) synthesizer while improving significantly on perceived naturalness relative to Flextalk. Each decision made during the research and development phase of this system was based on formal subjective evaluations. For example, the best voice found in a test that compared TTS systems built from several speakers gave a 0.3 -point head start (on a 5-point rating scale) in quality over the mean of all speakers. Similarly, using our Harmonic-plusNoise speech representation gave us a 0.25 -point advantage over standard TD-PSOLA. Finally, not performing prosodic modifications (other than some smoothing across concatenation points) on the units but using the system-generated prosody as a target in unit selection, 0.4 points were gained on overall quality. In conclusion, the new system combines the best of rule-based and data-driven worlds in TTS technology to deliver on the long-standing promise of truly natural-sounding synthesis.

\section{9:40}

2aSCa5. Domain-specific prominence-based concatenation. Thomas Portele (IKP, Univ. of Bonn, Bonn, Germany)

Some recent approaches to unlimited text-to-speech conversion do not use "designed" inventories of concatenative units like diphones. Instead, an annotated corpus of read speech is searched for the realization of a speech segment whose features best match the corresponding ones demanded by the synthesis input. Crucial for this approach are the size and the variety of the corpus, the number and kind of annotations, and the definition of "best match"' based on these annotations. In this implementation, the parameter "perceived prominence" is relied on as the most important parameter for the selection of a prosodically appropriate realization. A previous investigation with a German corpus indicated, however, that a corpus has to be very large in order to allow prosodic variations (e.g., different focus placements) of one utterance while retaining an acceptable intelligibility, if no post-selection signal modification is applied. The application of the same procedure to an American English corpus with domain-specific utterances (travel planning) indicates that the restriction to specific domains yields better results, but that signal manipulation is inevitable in order to obtain intelligible speech.

\section{0:00-10:20 Break}

\section{0:20}

2aSCa6. Phonetic transcriptions—Aligning canonical and pronunciation forms. Diane Hirschfeld (Tech. Acoust. Lab., Dresden Univ. of Technol., 01062 Dresden, Germany)

In database preparation for concatenative speech synthesis, the alignment of canonical and pronunciation form phone sequences plays a key role for further processing. Syllabification and word boundary detection are based on canonical transcriptions, but accent detection and phrasing is coupled to the speech signal and therefore to the pronunciation form. In text-to-speech processing, canonical transcriptions are available. Unit selection is done on the speech signal and has to take into account the realized phone sequence. If a good alignment of canonical and pronunciation form of the database is available, pronunciation variants are generated implicitly in unit selection. Finally, correct alignment of phone sequences is interesting for other disciplines, too (i.e., generation of pronunciation lexica for recognition). On the bases of manual alignment for our database of a male speaker of German, the most frequent deviations from the canonical form are categorized and assigned to syllable constituents and phone combinations. For automatic alignment, a system of articulatory/acoustic features of phone combinations is developed. Similarity measures are derived from this feature system for a given phone sequence. The presented algorithm is evaluated in relation to manual alignment results. 
2aSCa7. HMM-based database segmentation and unit selection for concatenative speech synthesis. Holzapfel Martin (SIEMENS AG ZT IK 5, 81739 Munich, Germany, Martin.Holzapfel@mchp.siemens.de)

In the proposed paper methods for improved HMM-based segmentation and unit selection for concatenative speech synthesis from a large corpus will be presented. The proposed algorithm is based on triphone HMMs. For training only the database to be segmented and its canonical phonetic transcription are used. Experiments on the optimization of these HMMs for segmenation are reported. Results in changes of the HMM topology, overadaption to the database, and variations on the acoustical features will be highlighted. Special attention is given to the tree-based clustering of the models according to their phonetic context. Contrary to other approaches, the clustering is performed on phone-model level. The clustering-tree is the basis of the phonetically motivated selection of the phone-sized speech units. Using the binary membership of those clusters as selection criterion results in a target conflict on the size of those clusters. (Tight phonetic description versus prosodic and contextual variety in one cluster.) Using the distance of the resulting nodes of the tree offers a still discrete but much finer scaled phonetic selection criterion overcoming the above-mentioned shortcomings. These techniques decreased the distances of the segmentation from manual labeling and significantly improved the overall quality of the Siemens TTS System PAPAGENO.

\section{1:20}

2aSCa8. Waveform models for data-driven speech synthesis. Michael W. Macon (Oregon Grad. Inst., 20000 NW Walker Rd., Beaverton, OR 97006)

Many "data-driven" models for synthesizing speech rely on concatenating waveforms extracted from a database. However, the number of perceptually important degrees of freedom in speech make it unlikely that enough data could be collected to cover all combinations of phonetic variables. By utilizing models that can transform the waveform in perceptually relevant ways, the space of acoustic features covered by the data can be expanded. The minimal requirement for such a model is parametric control of the fundamental frequency and duration. In addition to this, dimensions such as voice quality characteristics (breathiness, creak, etc.), phonetic reduction, and voice identity can be altered to expand the range of effects realizable from a given database. A few classes of models have been proposed to allow varying degrees of control over these dimensions. Trade-offs between flexibility, fidelity, and computational cost exist with each. This paper will describe common threads running through the best-known approaches, including handling of nonperiodic components and sensitivity to measurement errors. Although each of these approaches has its own merits, none has been proven to satisfy all the desired properties for synthesis simultaneously. The limits of some of the simplifying assumptions underlying these methods will be demonstrated, and areas needing improvement will be outlined.

11:40

2aSCa9. Concatenative synthesis with subwords for restricted-prosody applications. Marian Macchi and Dan Kahn (E-Speech Corp., Princeton, NJ)

For applications of text-to-speech in which the message prosody is restricted, very-high-quality speech can be achieved by concatenation of prerecorded words without physical modifications to the recorded words, if the words were spoken with the appropriate prosody. However, if the vocabulary is unlimited or changeable, it is necessary to have strategies for producing out-ofvocabulary words. Experiments were done with concatenating subword units from a prerecorded inventory to produce names (First name + last name pairs) spoken in isolation, a message set that is rich in phonemic and word-level prosody but extremely restricted in phrasal prosody. In the present approach, the use of rules predicting duration, pitch, and coarticulatory smooth was minimized. Those phonetic events that minimize distortion when functioning as splice points were identified, and then units were defined as the interval between these phonetic events. The units are labeled in terms of constituent phonemes, stress, word structure, intonation, and adjacent phonemes. Some of the labels were then collapsed into equivalence categories. Physical manipulations of the units were limited to time-domain smoothing of pitch at the concatenation boundary. Work is under way to optimize the set of splice points and maximize the size of the equivalence classes.

12:00-12:20 Discussion 


\title{
Session $2 \mathrm{aSCb}$
}

\section{Speech Communication: Cross Language and L2 Phonetics I}

\author{
James E. Flege, Cochair \\ Department of Rehabilitation Sciences, University of Alabama, Birmingham, Alabama 35294-0019, USA \\ Ocke-Schwen Bohn, Cochair \\ English Department, Aarhus University, DK-8000 Aarhus C, Denmark
}

Chair's Introduction-7:55

Invited Papers

8:00

2aSCb1. Spoken word recognition by non-native listeners. Ann R. Bradlow (Dept. of Linguist., Northwestern Univ., 2016 Sheridan Rd., Evanston, IL 60208, abradlow@ nwu.edu)

While the ability to perceive novel phoneme contrasts is necessary for accurate spoken word recognition by non-native listeners, it is likely that novel phoneme perception functions in a linguistically meaningful manner only once the contrast in question signals a known lexical contrast. In other words, knowledge of the sound-based structure of the target language lexicon is just as important for non-native speech perception as experience with the structure of the target language phoneme inventory. In order to understand non-native speech perception fully, there is a need to investigate recognition of word-sized units by non-native listeners using stimulus materials that are well controlled in terms of the sound-based structure of the target language lexicon. Accordingly, how non-native listeners recognize English words under conditions known to affect spoken word recognition by native English listeners was investigated. Results showed that the ability to take advantage of consistent surface phonetic information, such as a consistent talker across items, is a perceptual skill that transfers easily from first to second language perception. However, non-native listeners had particular difficulty with words that are easily confused with other similar sounding words, suggesting that non-native word recognition may be compromised when fine phonetic discrimation at the segmental level is required.

\section{8:20}

2aSCb2. The role of speaking rate in the perception of L2 speech. Murray J. Munro (Dept. of Linguist., Simon Fraser Univ., Burnaby, BC V5A 1S6, Canada, mjmunro@sfu.ca)

Previous work has shown that late second language (L2) learners typically speak more slowly than native speakers, and that speaking rate tends to correlate with overall L2 proficiency. However, the role of rate in native listeners' judgments of the global accentedness of L2 speech has not yet been established. Because rate tends to covary with proficiency, it is not known, for instance, whether the poorer global accent judgments typically assigned to slow L2 speech are due to rate differences or to other properties (such as segmental accuracy). This paper is a synthesis of the results of a series of experiments addressing this relationship. The effect of rate differences on evaluations of global accentedness of sentence-length utterances was assessed by compressing and expanding L2 speech and presenting it to listeners for accentedness ratings. Rate differences accounted for a small but significant portion of the variance in listeners' judgments. Overall, slightly accelerated L2 speech tended to be rated more highly than slower speech. These results are discussed in the context of rate measurements from a variety of types of L2 speech samples. The discrepancy between typical L2 production rates and native listeners' rate preferences is examined. [Work supported by SSHRC.]

\section{8:40}

2aSCb3. Toward further understanding of second language speech learning: An approach utilizing speech technology. Reiko Akahane-Yamada (ATR Human Information Processing Res. Labs., Kyoto, 619-0288 Japan, yamada@hip.atr.co.jp)

Recent training studies have clarified several aspects of second language (L2) learning. However, many questions still remain unanswered, and further training studies are necessary. In this paper, today's speech technology was applied to speech perception and production training. First, new speech resynthesis algorithm (STRAIGHT) was used to enhance training stimuli. Japanese speakers were trained to identify English /r/ and /l/ using several versions of synthetic stimuli with different manipulations. All the versions showed equivalent training effect as the original stimuli, suggesting that this resynthesis technique has a potential to enhance training materials. Second, two attempts were made to build an automated speech production training system, focusing on the issue of how to provide useful feedback for learners. Spectrogram and HMM-based speech recognition scores were attempted as feedback, and Japanese speakers were trained to produce /r/ and /l/. Subjects with either feedback improved significantly from pretest to posttest in their production ability as evaluated by human judges. A correlation was also found between evaluation scores obtained from HMM and human judges, further supporting the applicability of a HMM-based speech recognition system. Various approaches to optimize L2 speech training methods will be discussed in the context of the theories of speech perception/production development. 
2aSCb4. Non-natives' production of vowels in conversational speech. Thorsten Piske, James E. Flege (Dept. of Rehabilitation Sci., Univ. of Alabama, 503 Voker Hall, Birmingham, AL 35294-0019), Ian R. A. MacKay (Univ. of Ottawa, Ottawa, ON K1N 6N5, Canada), and Diane Meador (Speech and Lang. Sci. Res. Lab., Juneau, AK 99802)

The aim of this study was to determine if native speakers of Italian can ever learn to produce accurately English vowels not found in Italian. A recent study [J. E. Flege, I. R. A. MacKay, and D. Meador, J. Acoust. Soc. Am., under review], in which native Italian subjects were asked to repeat real words presented via a loudspeaker, showed that early but not late bilinguals produced English II $v \gamma /$ accurately. None of the subjects, however, did so when asked to repeat nonwords. But what about the most crucial evidenceconversational speech? Here a new technique with satisfactory experimental control ws developed to assess vowels produced in conversational speech. Speech samples were obtained from the same 90 subjects who participated in the earlier study. The subjects' production of /I $U$ r/ (not found in Italian) and /i æo o/ was evaluated auditorily by native English-speaking listeners. Preliminary results indicate that early bilinguals can learn to produce /I $U x /$ accurately in conversational speech, but that only a declining proportion of subjects do so as the age of learning English increases. High scores for /o/, which is much like an Italian vowel, were obtained. This suggested that listeners are not unduly influenced by overall degree of foreign accent in the samples examined.

\section{9:20}

2aSCb5. Age of learning affects the learning of $\mathbf{L 2}$ pronunciation but not morphosyntax. James E. Flege (Dept. of Rehabilitation Sci., VH503, Univ. of Alabama, Birmingham, AL 35294, jeflege@uab.edu), Grace Yeni-Komshian (Univ. of Maryland, College Park, MD 20742), and Serena Liu (Univ. of Alabama, Birmingham, AL 35294)

Many studies have shown that performance in a second language (L2) decreases as the age of L2 learning increases, but such findings are difficult to interpret because many factors are confounded with age. Here we assessed the overall degree of foreign accent in English sentences, and scores obtained on a 144-item grammaticality judgment test (GJT). The subjects were 240 Koreans who differed according to their age of arrival (AOA) in the United States ( 2 to 23 years). As AOA increased, foreign accents grew stronger and the GJT scores decreased steadily. However, when variables confounded with AOA were controlled using a subgroup matching technique, AOA was found to exert a significant effect on the foreign accent ratings but not on the GJT scores. Total years of education in the United States, but not language use or AOA, significantly affected scores for a subset of GJT items that tested rule-based, generalizable aspects of English morphosyntax. Conversely, language use, but not education or AOA, affected the scores for GJT sentences testing lexically based aspects of morphosyntax. Age effects on L2 pronunciation thus appear to be real. However, differences in morphosyntactic knowledge may instead be due to age-related variation in education or English-language use.

\section{9:40-10:00 Break}

\section{0:00}

2aSCb6. On what it takes to predict perceptual difficulty in cross-language vowel perception. Ocke-Schwen Bohn (English Dept., Aarhus Univ., DK-8000 Aarhus C, Denmark), Winifred Strange (CUNY Grad. Ctr., New York, NY), and Sonja A. Trent (Univ. of South Florida, Tampa, FL)

According to current models of cross-language speech perception and second language (L2) speech learning, perceptual difficulty depends upon how listeners map the phonetic segments of the foreign language onto their native language (L1) categories. A number of indirect methods have been suggested for predicting perceptual difficulty, e.g., contrastive phonemic analyses and acoustic comparisons of L1 and L2 sounds. This presentation summarizes recent studies which directly assessed perceptual assimilation patterns for North German vowels (as perceived by American English listeners) and for British English vowels (as perceived by Danish listeners). Vowels were produced in CVC syllables in various consonantal contexts in both citation form and sentence frames. As expected, contrastive phonemic analyses were not good predictors of assimilation patterns. Somewhat surprisingly, acoustic comparisons didn't always predict assimilation patterns either. In particular, measures of acoustic similarity from "canonical" forms didn't predict acoustic similarity for coarticulated vowels, nor did these measures predict perceptual similarity. Our results strongly suggest that perceptual difficulty in cross-language speech perception cannot be predicted using indirect methods, nor can difficulty be predicted by perceptual studies of citation form utterances. Instead, successful predictions of perceptual difficulty must be based on direct assessments of assimilation patterns. [Work supported by NIDCD.]

10:20

2aSCb7. Phonemic repertoire effects in lexical activation. Anne Cutler (MPI for Psycholinguist., P.O. Box 310, 6500 AH Nijmegen, The Netherlands)

Languages differ in the size and makeup of their phonemic repertoire. Phoneme-detection experiments have shown that speakers of languages with large, confusable vowel repertoires (English, Dutch) differ in their expectations of phonetic variability from speakers of languages with small distinct vowel repertoires (Spanish, Japanese). The present study asks whether these different expectations also constrain the activation of spoken words, via two word reconstruction experiments, in which listeners reconstruct from nonwords (e.g., eltimate) a real word by changing just one phoneme (ultimate, estimate). Dutch listeners responded significantly faster and made fewer errors when required to change vowels as opposed to consonants; when allowed to change any phoneme, they altered vowels significantly more often than consonants, and vowel responses were made more rapidly than consonant responses. This suggests that for these listeners, vowel information constrains lexical activation less tightly (allows more potential alternative candidates) than consonant information. In a language with few, distinct vowels, however, this weaker constraint of vowel information on lexical activation is not found: Spanish subjects, in the same task, responded equally rapidly and accurately whether required to alter vowels or consonants; when allowed to change any phoneme, they marginally preferred to alter consonants rather than vowels. 
2aSCb8. Native-language phonetic and phonological constraints on perception of non-native speech contrasts. Catherine Best (Dept. of Psychol., Wesleyan Univ., Middletown, CT 06459 and Haskins Labs, 270 Crown St., New Haven, CT 06511, best@ haskins.yale.edu)

Adults discriminate many non-native speech contrasts poorly, especially from an unfamiliar language that is not (yet) an L2. The Perceptual Assimilation Model (PAM) [Best et al., JEP: HPP 14, 34560 (1988)] posits that this difficulty stems from knowledge of both the phonological functions and the phonetic details of native speech segments. Thus, discrimination of an unfamiliar contrast depends not only on whether it resembles a native phonological contrast, but also on perceived goodness of fit between the non-native segments and native phonetic category(s). Cross-language comparisons support PAMs prediction that perceptual assimilation of contrasting non-native segments to a single native category yields poorer discrimination than assimilation to two categories (TC contrasts). Discrimination is worst if both phones are equally similar to a single category (SC), substantially better if they differ in category goodness (CG), near ceiling for TC assimilations, and good to excellent for consonants that fail to be assimilated as speech, instead being perceived as nonspeech events (non-assimilable: NA). Recent findings indicate that, as predicted, SC, CG, and TC assimilations are associated with preferential activation of left hemisphere language regions, whereas NA stimuli yields bilateral brain activation. Other findings with fluent bilinguals indicate a persisting L1 effect on non-native consonant discrimination even if their L2 is acquired prior to 5 years. [Work supported by NICHHD and NIDCD.]

\section{1:00}

2aSCb9. Cortical representation of language in monolinguals and bilinguals. Jacques Mehler (CNRS VA1198, 54 B. D. Raspail, Paris 75006, France)

A presentation will be made of experiments trying to evaluate language representation in monolingual and bilingual subjects. The impact of distance of the languages on the cortical representations of L1 and L2 will be thus evaluated. Furthermore, also considered will be the age at which L2 has been acquired and the degree of proficiency that the bilinguals have attained in L2. Data will be presented from PET, fMRI, MEG, and high-density evoked potentials. Results tend to show that level of proficiency is more important than age of acquisition to explain the cortical representation of L2. Comparing and contrasting standard psycholinguistic evaluations with imaging studies, the respective advantages and shortcomings will be discussed. In the second part of the presentation the hypothesis that languages can be arranged into a limited number of classes will be explored. A review of work showing that infants discriminate a change of language only if the test and habituation languages have different rhythmical properties will be presented. The notion that languages can be sorted into rhythmical classes can be evaluated experimentally and results will be presented.

\section{1:20}

2aSCb10. Research in cross-language and second-language speech perception and production: Issues, trends, and future directions. Winifred Strange (Speech and Hearing Sci., CUNY Grad. School, 33 W. 42nd St., New York, NY 10036-8099)

Theoretical and methodological advances presented in the preceding papers of this special session will be summarized and critiqued. Suggestions for future research will be offered. The comments offered in this talk are intended to generate discussion by the participants and audience of the special session.

11:40-12:20 Discussion 


\title{
Session 2aSP
}

\section{Signal Processing in Acoustics: Acoustic Image Synthesis for Arbitrary Listeners}

\author{
Anthony J. Brammer, Cochair \\ IMS/ASP, National Research Council, M-36 Montreal Road, Ottawa, Ontario K1A OR6, Canada \\ Adelbert W. Bronkhorst, Cochair \\ Perception Department, TNO Human Factors Research Institute, P.O. Box 23, 3769 ZG Soesterberg, The Netherlands
}

\author{
Chair's Introduction-7:55 \\ Invited Papers
}

2aSP1. Are individualized head-related transfer functions required for auditory information displays? Elizabeth M. Wenzel (NASA Ames Res. Ctr., M.S. 262-2, Moffett Field, CA 94035-1000, bwenzel@mail.arc.nasa.gov) and Durand R. Begault (San Jose State Univ., Moffett Field, CA 94035-1000)

In aerospace applications, spatial cues in auditory displays can allow an astronaut, ground-controller, or other human operator to take advantage of their natural ability to localize sounds in three-dimensional space. Synthetic localization of acoustic images can be used to enhance situational awareness, improve segregation of multiple audio signals through selective attention, and provide a means of detecting a desired signal against noise for enhanced speech intelligibility. A number of studies in the literature have suggested that individualized head-related transfer functions (HRTFs) produce the best localization performance for a given listener in a spatial auditory display. However, it is often impractical to measure the HRTFs for each potential user of an aerospace display. Further, whether or not individualized cues are required may depend upon the nature of the task that is being aided. For example, a communications display may not require individualized cues to enhance speech intelligibility, while a display that represents the direction of incoming aircraft to an air traffic controller may benefit from individualized HRTFs. This paper describes some of the spatial auditory displays that have been investigated at NASA Ames and discusses the efficacy of using nonindividualized cues in the context of various aerospace tasks.

8:20

2aSP2. Localization in real life and with individual, nonindividual, and artificial head binaural recordings. Henrik Moller (Acoust. Lab., Aalborg Univ., Fredrik Bajers Vej 7 B4, DK-9220 Aalborg O, Denmark)

Human sound localization was studied in real life (1), and with binaural recordings made in the ears of the listener him/herself (2), in the ears of other humans (3), and with artificial heads (4). The localization with individual binaural recordings turned out to be identical to that of real life. An increased number of errors was seen for nonindividual binaural recordings, especially in terms of confusions between median plane sources. It proved possible to select human "recording heads" that offered recordings which gave more favorable results than others. The highest number of errors was seen with artificial head recordings. This is surprising, since the artificial heads aim at replicating the acoustics of the "average"' human. Measurements of head-related transfer functions confirmed differences between data obtained with artificial heads and those obtained from humans. The paper gives an overview of experiments and measurements carried out at Aalborg University over a number of years.

8:40

2aSP3. Relearning sound localization with new ears. P. M. Hofman, A. J. Van Opstal, and J. G. A. Van Riswick (Dept. of Medical Phys. and Biophys., Univ. of Nijmegen, Geert Grooteplein 21, NL-6525 EZ Nijmegen, The Netherlands)

Because the inner ear is not organized spatially but tonotopically, sound localization relies on the neural processing of implicit acoustic cues. To determine a sound's position, the brain must learn and calibrate these individual cues, using accurate spatial feedback from other sensorimotor systems. Experimental evidence for such a system has been demonstrated in barn owls, but not in humans. In this study, the existence of ongoing spatial calibration in the adult human auditory system is demonstrated. The spectral elevation cues of human subjects were disrupted by modifying their outer ears (pinnae) with molds. Although localization of sound elevation was dramatically degraded immediately after the modification, accurate performance was steadily reacquired. Interestingly, learning the new spectral cues did not interfere with the neural representation of the original cues, as subjects could localize sounds with both normal and modified pinnae. 
2aSP4. Explaining individual differences in head-related transfer functions. Frederic L. Wightman and Doris J. Kistler (Waisman Ctr., Univ. of Wisconsin, 1500 Highland Ave., Madison, WI 53705, Wightman@waisman.wisc.edu)

The fidelity of virtual auditory images is degraded by differences between the listener's head-related transfer functions (HRTFs) and the HRTFs used to synthesize the virtual images. Middlebrooks (this meeting) argues that simple frequency scaling accounts for many of these individual differences. In an attempt to understand more fully both the capabilities and limitations of frequency scaling, the HRTFs from 100 individuals were analyzed. The difference between each pair of HRTFs was expressed as a single number, the average of the squared $\mathrm{dB}$ differences at $40 \mathrm{log}$-spaced frequencies from 0.12 to $12 \mathrm{kHz}$. A multidimensional scaling analysis (ALSCAL) was performed on the resulting difference matrix. A two-dimensional solution accounted for over $80 \%$ of the total variance. The frequency scaling factor needed for the optimal fit of each individual's HRTF set to the HRTF set at the center of the 2-D solution was then determined. The correlation between the optimal frequency scalar and the coordinate of each HRTF set on one dimension of the scaling solution was over 0.95. Attempts to reveal a physical basis for the second dimension have been less successful. [Work supported by NIH, NASA, and ONR.]

9:20

2aSP5. Adapting head-related transfer functions to individual listeners. Adelbert W. Bronkhorst (TNO Human Factors Res. Inst., P.O. Box 23, 3769 ZG Soesterberg, The Netherlands, bronkhorst@tm.tno.nl)

A highly efficient data reduction scheme will be described that captures the interindividual variability, present in head-related transfer functions (HRTFs), in only a limited number of parameters. The techniques used are principal component analysis and least-squares fitting of orthogonal spherical functions. The distortions introduced by the data reduction were evaluated using a distance measure based on the $\mathrm{dB}$ differences between HRTFs within $1 / 6$ octaves. The distance measure correlates strongly with results of listening tests in which original HRTFs were compared with HRTFs reconstructed from the compressed data. Adaptation of HRTFs to an individual can be performed by reconstructing a set of HRTFs using estimated parameter values. Two methods for estimating these values will be discussed: prediction based on anthropometrical data and optimization using a simple listening test.

9:40-10:00 Break

\section{Contributed Papers}

\section{0:00}

2aSP6. The contribution of spectral cues to human sound localization. Erno H. Langendijk and Adelbert W. Bronkhorst (TNO Human Factors Res. Inst., Kampweg 5, 3796 DE Soesterberg, The Netherlands, erno@tm.tno.nl)

The contribution of spectral cues to human sound localization was investigated by removing cues in 1/2-, 1-, or 2-octave bands in the frequency range above $4 \mathrm{kHz}$. Localization responses were given by placing an acoustic pointer at the same apparent position as a virtual target. The pointer was generated by filtering a 100-ms harmonic complex with equalized head-related transfer functions (HRTFs). Listeners controlled the pointer by a hand-held stick that rotated about a fixed point. In the baseline condition the target, a 200-ms noise burst, was filtered with the same HRTFs as the pointer. In other conditions the spectral information within a certain frequency band was removed by replacing the directional transfer function within this band with the average transfer of this band. Analysis of the data showed that the effect of bandwidth is larger than that of center frequency. There appears to be an interaction between center frequency and target position. Effects related to center frequency varied substantially among listeners. This indicates that listeners use different cues when localizing sounds from a certain direction.

\section{0:20}

2aSP7. Sound localization using other people's ears. John C. Middlebrooks (Kresge Hearing Res. Inst., Univ. of Michigan, 1301 E. Ann St., Ann Arbor, MI 48109-0506, jmidd@umich.edu)

When subjects attempt to localize virtual targets that have been synthesized with directional transfer functions (DTFs) recorded from other subjects' ear canals, they typically show a considerable increase in localization error relative to the condition in which DTFs are recorded from their own ear canals. This study explored intersubject differences in DTFs and developed a procedure to reduce such differences, thereby improving virtual localization. DTFs varied systematically among subjects in regard to the center frequencies of spectral features such as peaks and notches. Generally, spectral features appeared at lower frequencies in the DTFs of larger subjects and at higher frequencies in DTFs of smaller subjects.
Characteristic errors in virtual localization could be related to frequency differences between spectral features in the listener's own DTFs and those with which virtual targets were synthesized. Scaling transfer functions in frequency by an optimal scalar could reduce intersubject variance in DTFs, sometimes by more than $50 \%$. Frequency scaling of DTFs resulted in corresponding improvements in virtual localization. In the majority of cases, optimal frequency scaling reduced by more than half the increase in error that resulted from listening through another subject's ears. [Work supported by ONR Grant No. N00014-94-0481 and NIH Grant No. DC00420.]

\section{0:40}

2aSP8. Holophony versus ambisonic: Deriving a hybrid method for 3-D sound reproduction in videoconferencing. Rozenn Nicol and Marc Emerit (France Telecom CNET, 2 Ave. Pierre Marzin, 22307 Lannion Cedex, France, rozenn.nicol@ cnet.francetelecom.fr)

Current research in videoconferencing intends to introduce 3-D reproduction methods both for image and sound. This paper will deal only with the problem of 3-D sound reproduction. First, one specific requirement must be borne in mind: in a videoconference, the listening area has to be wide, since it is addressed to several listeners. Two approaches have been considered: Holophony-acoustical equivalent to holography-and ambisonic. In a previous paper, it has been shown that these two methods are based on the same principle, i.e., physical reconstruction of the acoustical 3-D field, which appears as the most relevant solution to obtain an extensive listening area. However, holophony and ambisonic achieve opposite compromises between system complexity (number of signals recorded, number of microphones and loudspeakers) and reconstruction performance (accuracy, size of listening area, implementation opportunities). The two approaches are compared in order to point out a hybrid method, which optimizes the result. First studies focus on sound pick-up and show that ambisonic is more efficient concerning the coding of spatial information. In this paper, this analysis will be completed, first by examining the sound reproduction step, and second by studying the method robustness toward the actual behavior of transducers. 


\title{
Session 2aUWa
}

\section{Underwater Acoustics, Acoustical Oceanography, and Physical Acoustics: Acoustics and the Comprehensive Test Ban Treaty I}

\author{
Arthur B. Baggeroer, Cochair \\ Department of Ocean Engineering, Massachusetts Institute of Technology, 77 Massachusetts Avenue, Cambridge, \\ Massachusetts 02139, USA \\ Martin W. Lawrence, Cochair \\ Provisional Technical Secretariat, Comprehensive Nuclear-Test-Ban Treaty Organization, Vienna International Centre, \\ P.O. Box 1200, A-1400 Vienna, Austria
}

Invited Papers

2aUWa1. Overview of the hydroacoustic monitoring system for the Comprehensive Nuclear-Test-Ban Treaty. Martin W. Lawrence (Provisional Tech. Secretariat, Comprehensive Nuclear-Test-Ban Treaty Organization, Vienna Intl. Ctr., A-1400 Vienna, Austria,mlawrence@ctbto.org)

A global hydroacoustic monitoring system is being implemented for use in verification of the Comprehensive Nuclear-Test-Ban Treaty (CTBT). This system will provide hydroacoustic monitoring of all the world's oceans for $24 \mathrm{~h}$ a day, every day of the year, into the indefinite future. This unique resource will utilize two types of station. One type will be based on a hydrophone at the SOFAR axis depth, cabled back to shore. The other will be based on a seismometer on a small island using detection of the T-phase signal. This latter station relies on a signal which has propagated predominantly through the ocean, but has been converted to seismic energy at the margin of the island. This new application of hydroacoustics highlights a number of scientific questions which have not been adequately addressed. Progress in implementation of this network will be described.

8:40

2aUWa2. Acoustic source calculations for nuclear bursts. Douglas B. Clarke, Philip E. Harben, and David B. Harris (Lawrence Livermore Natl. Lab., Livermore, CA)

Research has been conducted on the source term for long-range underwater propagation of signals from nuclear explosions in and above the ocean, in support of CTB monitoring objectives. A suite of source-region simulations is reviewed to study the variation of wave properties and source-region energy partition as a function of height or depth of burst. The multistep calculation combines LLNL's CALE hydrodynamics code in the strong shock region with NRL's weak shock code, NPE, at intermediate ranges. The source term calculations are intended as a starter field for long-range linear propagation models to obtain signature estimates at normal observational ranges. Calculations are presented to examine the effect of sea ice below an air burst on acoustic coupling into the water column. The ice was modeled as a continuous elastic layer 5-m thick at the water surface, and the source as a 1-Kt explosion $50 \mathrm{~m}$ above the ice. Calculations with and without the ice layer predict a moderate, but noticeable reduction in coupled acoustic energy in signals observed at the 10000-m range. [Work performed under the auspices of the U. S. Department of Energy by the Lawrence Livermore National Laboratory under Contract No. W-7405-ENG-48.]

9:00

2aUWa3. Hydroacoustic monitoring of the oceans. Y. Caristan (CEA/DASE, BP. 12, 91680 Bruyers Le Chatel, France, caristan@dase.bruyeres.cea.fr)

In order to verify compliance with this Treaty, an international Monitoring System based on four technologies, seismology, infrasounds, hydroacoustic, and radionuclides, shall be implemented. Therefore, a network of hydrophones and seismic T-wave stations has been designed and evaluated in terms of detectability and location capability. Hydroacoustic waves generated by an undersea explosion or by oceanic earthquakes are propagating over several thousands of kilometers within an oceanic waveguide called So.F.A.R., channel (sound fixing and ranging) as shown by Ewing and Tolstoy (1950). When they are reaching any continental slope or island they are converted in seismic T-waves which are recorded by seismographs. By using both numerical simulations and experimental analysis, a methodology has developed which allows to better understand and quantify the effects of the source parameters and of the propagation characteristics (specifically on the So.F.A.R. channel and of the continental slope) on the recorded waveforms. The results obtained should be useful to improve the evaluation of detectability and location capability of the future hydroacoustic network and should provide some effective guidance to the site survey process. For that purpose and also to have a better understanding of the source parameters and of the acoustic wave propagation, a methodology has developed using both a numerical simulation and an experimental analysis. 
2aUWa4. Hydroacoustics in monitoring the comprehensive test ban treaty. John A. Orcutt (Inst. of Geophys. and Planetary Phys. (0225), Scripps Inst. of Oceanogr., La Jolla, CA 92093, jorcutt@igpp.ucsd.edu)

The Comprehensive Test Ban Treaty was signed by the U. S. in 1996 and many other nations have since joined in supporting the treaty. On a planet which is more than $70 \%$ covered by oceans, only 11 sites will monitor acoustic propagation in the oceans. The capabilities of the system are further limited through the avoidance of any directionality capability at the sites and five of the sites are simply seismometers located on islands. Several research projects become very important in developing understanding to the level needed to remove some of the more substantial problems introduced by this limited capability. In particular, the coupling of acoustic to seismic wave propagation in the vicinity of islands is an important issue. Research has demonstrated that the efficiency of coupling, for example, is highly dependent on azimuth and, hence, the offshore morphology. Additional work is required to develop a predictive capability for coupling. Typically hydrophones in the ocean detect far more events than seismometers on land. This, coupled with the slow speed of propagation, makes location of events difficult. Research involving the joint location of events using hydrophones and seismometers will be reviewed.

9:40

2aUWa5. Automated detection of underwater explosions by the IMS hydroacoustic network. Hyrum Laney, Paul Dysart, Herbert Freese (Science Applications Intl. Corp., 1710 Goodridge Dr., McLean, VA 22102, dysart@osg.saic.com), Douglas Brumbaugh, Ronan Le Bras, and Jeffrey Hanson (Science Applications Intl. Corp., San Diego, CA 92121)

An important component of the CTBT monitoring system is the 11-station hydroacoustic network designed to monitor the oceans for unannounced underwater explosions. Explosions that do not breach the sea surface consist of a series of pulses due to gas bubble oscillations. The period of oscillation is a well-known function of the yield and detonation depth for explosives up to several thousand kilograms of TNT equivalent. The presence of a bubble pulse is a strong indicator of an explosive source and, therefore, is useful in event characterization. Examples of underwater explosions, which can be used to calibrate signal processing algorithms and propagation models, are rare. A set of explosive and other impulsive events recorded on hydrophones at Wake Island and Point Sur, CA is presented. These include the French nuclear tests, volcanic events, and chemical explosions. The set also includes pressure time-series simulated using a normal-mode propagation model. The automatic signal processor has been developed to search the real cepstrum for peaks using a noise-spectral equalization algorithm similar to those used in passive sonar. Examples illustrate complications due to range, depth, ambient noise, and waveguide distortion.

10:00-10:20 Break

10:20

2aUWa6. Characterization of reflected hydroacoustic signals for ctbt localization. Jeffrey Angell, Ted Farrell, and Jay Pulli (GTE/BBN Technologies, Arlington, VA 22209)

The sparse nature of the proposed IMS hydroacoustic network implies that maximizing the information from each sensor is critical to overall network performance for nuclear event localization. Performance may be enhanced using information contained in late-time reflected arrivals from continental shelves, islands, and seamounts. The primary focus here is to characterize reflected signals and determine the conditions under which reflections are likely to occur. Several data sets, including a 1970 ship-scuttling explosion conducted in the North Atlantic (CHASE 21 event) are analyzed. Travel-time ellipses and bearing estimates generated from measured arrival times of waveform envelopes are used to determine possible geographic locations of acoustic reflections. Environmental characteristics at these locations are examined in order to understand the conditions under which reflections are likely to occur. Timeand frequency-domain characteristics of both the direct arrivals and reflections are measured and discussed. [Work sponsored by Defense Special Weapons Agency Contract Number DSWA01-97-C-0164.]

\section{Contributed Papers}

10:40

2aUWa7. Modeling hydroacoustic waveform envelopes for comprehensive test-ban treaty monitoring. Yevgeniy Y. Dorfman (BBN Technologies, 70 Fawcett St., Cambridge, MA 02138) and Jay J. Pulli (BBN Technologies, Rosslyn, VA 22209)

Robust and accurate localization of explosion events in the oceans requires modeling signal arrival times for a number of receivers. Additional information contained in the waveform envelope may also be used to recover other source properties and reduce the probability of false alarms in nuclear explosion monitoring. The feasibility of efficient envelope modeling was investigated by comparing adiabatic normal-mode model predictions with explosion data. The KRAKEN computer code, combined with appropriate dispersion curve interpolation, was used to compute modal shapes and wave numbers in each range segment. To facilitate CTBT knowledge base development, the model has been used to illustrate the various factors that contribute to the shape of the received hydroacoustic waveform envelope, and provide design feedback for sensor placement and data analysis algorithms. The model was compared with data for two source-receiver paths: (a) the Chase 21 ship scuttling explosion on 25 June 1970 off the New Jersey coast and recorded at the Ascension Island hydroacoustic array; and (b) nuclear explosions on Mururoa Atoll and received at the hydrophone at Point Sur, California. Adiabatic normal-mode approach was found inadequate for source and receiver located far from the SOFAR axis. [Work supported by U.S. DOE, Office of Nonproliferation and National Security.]

\section{1:00}

2aUWa8. Propagation modeling of $T$-phase time series via RDOASES. Brian Sperry (MIT/WHOI Joint Prog. in Oceanogr. and Ocean Eng., Woods Hole Oceanogr. Inst., Woods Hole, MA 02543), Henrik Schmidt, and Arthur Baggeroer (MIT, Cambridge, MA 02139)

Properly situated earthquakes and explosive sources often radiate acoustic energy into the oceanic waveguide and are thereby detectable at long ranges. Details of how this energy actually couples into the ocean remain elusive. Recent development of a range-dependent version of OASES has made it possible to model acoustic propagation in range- 
varying, elastic environments. It has been very successful in investigating the effect of epicenter depth for $T$-phase excitation on a sloping bottom. The present study extends earlier work to consider time-domain characteristics of the arrival and their dependence upon properties of the source and its surrounding region. The general environment considered here is a Munk sound-speed profile and sloping bottom out to a range of $30 \mathrm{~km}$, after which the bottom depth is constant. The seismoacoustic field is computed using RDOASES, and then projected onto the local mode shapes. The complex mode coefficients are propagated to a distant receiving array, where the time-domain signal is synthesized. Understanding the source excitation in terms of mode and frequency content is critical to interpreting the arrival structure. At teleseismic ranges, the dependence of group delays on mode number and frequency leads to significant time spreading of the various signal components.

\section{1:20}

2aUWa9. Normal-mode composition of earthquake $\mathbf{T}$ phases recorded in the deep ocean. Gerald L. D'Spain, Lewis P. Berger, William A. Kuperman (Scripps Inst. of Oceanogr., La Jolla, CA 92093-0704), Jeff L. Stevens, and G. Eli Baker (Maxwell Technologies, San Diego, CA 92123-1506)

For upslope propagation in an ocean environment, the place(s) where underwater acoustic field energy couples into the land seismic field is determined by the local water depth and the normal-mode composition of the acoustic energy, to first approximation. Therefore, the use of earthquake-generated $\mathrm{T}$ phases as natural probes of water-to-land coupling characteristics is aided by knowledge of their modal makeup. During the 1989 VAST experiment, conducted in 5000-m-deep water in the midlatitude Northeast Pacific Ocean (34.0 deg N, $140.0 \mathrm{deg}$ W), the Marine Physical Lab deployed a 200-element, 3000-m-aperture vertical hydrophone array from R/P FLIP. Several earthquakes generated detectable $\mathrm{T}$ phases during the 11-day recording period and the modal composition of five such events, including one on land, have been determined. Because of the extended nature of the T-phase source region, the individual modes are uncorrelated, permitting an eigenanalysis of the data cross spectral matrix at a given frequency to provide approximate mode amplitude and eigenfunction information. Results indicate that for the predominant T-phase energy around $5 \mathrm{~Hz}$, the first four or five modes are important, with the mode having largest amplitude often not being the lowest one. [Work supported by ONR and DSWA.]

\section{1:40}

2aUWa10. Progress in the implementation of the T-phase subnetwork for monitoring the oceans. Marta Galindo Arranz (Provisional Tech. Secretariat, Comprehensive Nuclear-Test-Ban Treaty Organization, Vienna Intl. Ctr., A-1400 Vienna, Austria, mgalindo@ctbto.org)

For the first time seismic technology is being used for monitoring the oceans, detecting and evaluating acoustic signals. Five seismic stations, called T-phase stations, are being established to complement six hydrophone stations, thus constituting the hydroacoustic international monitoring network. This T-phase subnetwork is mostly localized in the northern hemisphere and has been designed to work in synergy with the seismic network. Many technical difficulties arise at the time of installing new T-phase stations, due to lack of some relevant scientific knowledge. The T-phase stations are located on small islands with abrupt bathymetric properties and an open view of the ocean. Within this environment it is necessary to find the optimum distance from the coastline in order to achieve a compromise between sea-induced noise and good T-phase detection capability. Other open questions include the details of how acoustic energy is coupled into seismic energy, as well as how factors such as location and seasonal variations of the SOFAR channel affect the acousticto-seismic coupling. These questions are investigated at each site survey, upgrading, or new installation within the subnetwork. Results of these investigations will be presented as well as possible implications for technical specifications of the T-phase stations.

\section{2:00}

2aUWa11. Perturbative eigenvalue techniques for global-scale hydroacoustic pulse propagation. Gregory J. Orris, John S. Perkins, and Laurie T. Fialkowski (Naval Res. Lab., Washington, DC 20375)

Because of the computational difficulties associated with threedimensional acoustic propagation on global scales in the ocean, current models require a hybrid approach. In the Global-Scale Ocean Acoustic Raytrace Model, horizontal refraction of eigenrays is accomplished by first separating the vertical dependence of the field from the horizontal dependence and solving for the vertical eigenvalues and eigenmodes. This approximation is valid as long as the ocean medium is adiabatic in the horizontal direction. The eigenvalues and eigenmodes directly affect the local phase speed and group velocity, and consequently, refraction and travel time of a given ray. If Fourier synthesis is used in conjunction with another full-field method to obtain time-domain results along a horizontally refracted ray, then the computing requirements of determining the local eigenvalue spectrum as a function of frequency can become overly burdensome. An approach based on perturbation methods is introduced that can be used to compute the eigenvalue spectrum quickly at frequencies close to those at which the spectrum has already been determined, yet far enough away in frequency to be useful for the Fourier synthesis of pulse propagation. [Work supported by the U.S. Department of Energy, Office of Non-proliferation and National Security, Office of Research and Development, Contract No. DE-A101-95IS20011.] 


\title{
Session 2aUWb
}

\section{Underwater Acoustics: Rapid Ocean Environmental Assessment}

\author{
Ronald A. Wagstaff, Cochair \\ Code 7183, Naval Research Laboratory, Stennis Space Center, Mississippi 39529-5004, USA \\ Jürgen Sellschopp, Cochair \\ SACLANT Undersea Research Center, Viale San Bartolomeo 400, 19138 La Spezia, Italy \\ Invited Papers
}

\begin{abstract}
8:00
2aUWb1. Rapid response, a demonstration of ocean assessment capabilities. Jürgen Sellschopp and Tuncay Akal (SACLANT Undersea Res. Ctr., Viale San Bartolomeo 400, 19138 La Spezia, Italy)

The suitability of sound waves as a carrier of information in the ocean is known to be highly variable. In the past, the assessment of the controlling environmental parameters has typically been subject to post-cruise analysis and often took years. SACLANT Undersea Research Centre and NATO's organization for military oceanography agreed upon a three-year series of surveys in the Mediterranean Sea aimed at the development of a methodology for rapid environmental assessment (REA). A large number of research ships and patrol aircraft were provided by various nations. Researchers of numerous institutes were engaged in the REA trials. Time-invariant parameters such as bottom properties were measured directly or determined by inversion. The results were transferred for optional replacement in a standard database. Time-dependent parameters such as the sound velocity structure were predicted by means of real-time ocean models initialized and updated with survey data. The infrastructure for timely delivery of REA products and forecasts was developed, the main requirements being appropriate data channels and data fusion capacity. Rapid response was the first demonstration by the research community that the critical characteristics of the acoustic environment can be provided by the end of a mission.
\end{abstract}

8:20

2aUWb2. Rapid assessment of undersea ambient noise. Ronald A. Wagstaff (Naval Res. Lab., Stennis Space Center, MS 39529-5004) and Reginald D. Hollett (SACLANT Undersea Res. Ctr., La Spezia, Italy)

Undersea ambient noise is a critical parameter in determining the performance of passive sonar systems, and sometimes active systems. There are many properties of the ambient noise that must be considered in a rapid ambient noise field assessment, including mean and percentile levels as a function of frequency, temporal and spatial variation, vertical and horizontal directionality, and depth dependence. In addition, rapid interpretation and extrapolation of the measured and analyzed ambient noise results require a credible onboard ambient noise prediction model and collateral noise model input measurements of oceanographic, acoustic, meteorological, and noise source parameters, e.g., sea and swell height, wind speed, and the positions, speeds, courses, and lengths of near and distant ships. Finally, turnkey data processing programs and time proven analysis procedures must be utilized with onboard graphics and word processing capabilities to generate the assessment document onboard in final form. The methods and techniques used by the SACLANT Undersea Research Centre (SACLANTCEN) and the Naval Research Laboratory (NRL) for rapid onboard assessment of the ambient noise are presented and discussed. [Work supported by Project 01 of SACLANTCEN and the Office of Naval Research through the management of the WISPR Filter Development and Evaluation Project by NRL.]

8:40

2aUWb3. Summary of bottom reverberation findings and model/data comparisons during three rapid environmental assessment trials. John R. Preston (ARL, Penn State Univ., P.O. Box 30, State College, PA 16804) and Dale D. Ellis (Defence Res. Establishment Atlantic, Dartmouth, NS B2Y 3Z7, Canada)

From August 1996 through February 1998 the authors participated with SACLANTCEN in three rapid environmental assessment (REA) trials, including experiments for bottom reverberation. Eight sites were visited: three sites south of Sicily, three sites in the Ionian Sea, and two sites in the Atlantic near the Straits of Gibraltar. SUS charges were used as sources. The receivers were a nested horizontal array with 128 elements spaced at $0.5,1$, and $2 \mathrm{~m}$ and a second horizontal array with 32 elements spaced at $0.18 \mathrm{~m}$. Sets of nearly monostatic recordings were analyzed in frequency bands from 80 to $4000 \mathrm{~Hz}$. Polar plots of the beam time series were superimposed on bathymetric charts, revealing a number of scattering features not on the charts. The results illustrate the use of directional reverberation measurements as a useful remote-sensing tool. Another objective of the reverberation experiments was to quickly invert for estimates of scattering and geo-acoustic parameters that can be used in a wider variety of conditions. The horizontal array data were compared with the generic sonar model (GSM) predictions for selected cases. A summary of the model parameters found at the eight sites is presented. [Work partially supported by ONR.] 
2aUWb4. Shallow-water REA using autonomous ocean sampling networks. Henrik Schmidt, James G. Bellingham (MIT, Cambridge, MA 02139), and Allan Robinson (Harvard Univ., Cambridge, MA 02138)

Environmental management and naval operations in coastal waters are severely limited by a lack of oceanographic predictive capability associated with the strong spatial gradients and temporal variability inherent to such environments. A key to improving the predictive capability is a measurement system which can provide data in close to real time for assimilation into oceanographic circulation models. In that regard, the Autonomous Ocean Sampling Network (AOSN) combines small, autonomous underwater vehicles (AUV) with an underwater communication and navigation network to provide an ocean measurement system with real-time data access and adaptive sampling capabilities. The acoustic communication capability of the AOSN allows for feedback to the sensor platforms of uncertainties of the field estimates, information which can then be used to redistribute the AOSN resources optimally. The development of a new observation and prediction capability combining the AOSN with efficient coastal circulation models is the objective of an ongoing multidisciplinary research effort, but the feasibility has already been demonstrated through a series of field deployments. The results of these experiments are reviewed, and the potential for this new rapid environmental assessment capability for littoral environments is discussed.

9:20

2aUWb5. Rapid assessment of bottom backscattering. Tuncay Akal and Anthony P. Lyons (SACLANT Undersea Res. Ctr., Viale San Bartolomeo 400, 19138 La Spezia, Italy)

There is a strong need to obtain information about the expected acoustic reverberation environment that will be encountered in various shallow-water sites to aid in planning and evaluating the effectiveness of operational systems. A new technique is being developed at the SACLANT Undersea Research Centre to quickly sense the mean reverberation levels of a shallow-water area. A prototype device and analysis algorithms have been developed and used as part of rapid response operations. The system transmits and receives acoustic signals from an $80-\mathrm{kHz}$ transducer and simultaneously calculates the quantitative acoustic response of the seafloor. The reverberation measurements made so far have concentrated on mapping the mean seafloor scattering level (backscattering strength) and amplitude statistics over an area for various grazing angle regimes. Differences in scattering strength between high and low reverberation zones within an operational area were significant, with differences in mean level reaching 10-12 dB. Additionally, the reverberation measurements were compared with other techniques to verify relevant bottom-type classification. The experimental technique and data obtained from different areas are presented.

\section{9:40}

2aUWb6. In-situ update of geoparameter databases. Charles Holland, John Osler, Edward McDonald, and Carlo Ferla (SACLANT Undersea Res. Ctr., 19138 La Spezia, Italy, holland@saclantc.nato.int)

Low- and mid-frequency system performance is often controlled and degraded by bottom interaction, especially in littoral regions. However, performance prediction models generally give unreliable estimates of bottom interaction. The poor estimates are most often due to the databases that drive the models rather than the model physics. In this study a new approach is taken in rapid environmental assessment for overcoming this problem. The concept is to use in-situ propagation data to extract geoacoustic information about the bottom which is then used to update the bottom database. An analogous approach has long been employed for the water column, where in-situ bathythermograph (BT) data are used to update historical databases. While techniques for screening/merging BT data are now under development, there are no comparable techniques for treating bottom geoacoustic data. In this paper, a high-level methodology for updating bottom databases with in-situ measurements is outlined. The methodology includes a series of critical questions sequenced to lead to decisions about the robustness of the historical databases in a given region and the potential for database update. Results of the methodology, i.e., the in-situ measurements, modeling, and resulting bottom geoparameter database updates, from several littoral areas, will be shown.

10:00-10:20 Break

\section{Contributed Papers}

\section{0:20}

2aUWb7. Preliminary results from at-sea tests of the Autonomous Buoyed Environmental Sensor System (ABES). J. Mark Stevenson (Acoust. Branch, Space and Naval Warfare Systems Ctr., San Diego, CA) and John L. Newton (Ocean Sensors, Inc., San Diego, CA)

ABES is an autonomous oceanographic measurement system which provides relatively dense spatial and temporal sampling of the highly variable shallow-water ocean environment. Compared to more traditional sampling methods, ABES is low cost; albeit providing slightly lower measurement accuracy and resolution. ABES is not intended to replace existing high-resolution oceanographic instrumentation, but fill the niche for a low-cost, lower-resolution system that would provide higher spatial sampling. The instrument is a sensor string connected to a cylindrical electronics canister. The prototype sensor string contains one electromagnetic current meter, three tilt sensors, three magnetoresistive compasses, and ten conductivity, temperature, and depth sensors. These sensors are distributed along a 40-m cable. A system controller, GPS receiver, and spread- spectrum radio are housed in the 1-m-long electronics canister. Together, these components weigh $10 \mathrm{~kg}$ in air and occupy a volume of 51 . Data collected during three at-sea tests in 1998 and compared to traditional CTD measurements suggest that ABES can effectively define the temporal and spatial characteristics of the shallow-water environment. [Work supported by ONR 321-SS and 322-PO.]

\section{0:40}

2aUWb8. Estimation of modal eigenvalues from an arbitrary distribution of receivers in a planar array. Subramaniam D. Rajan (Sci. Solutions, Inc., 18 Clinton Dr., Hollis, NH 03049)

Acoustic propagation in shallow-water areas is strongly influenced by the acoustic properties of the sediment layers. Many approaches have been proposed for obtaining the acoustic properties of the sediment layers. A simple and powerful approach has been the inversion algorithms based on modal eigenvalues [Rajan et al., J. Acoust. Soc. Am. 82, 998-1017 (1987)]. The performance of this approach has been demonstrated in field 
experiments. In these field experiments, the data were obtained on a linear synthetic aperture horizontal array. In recent years there has been an effort to extend the procedure to cover wider areas so that the range-dependent properties of the sediment can be determined. A means of achieving this objective is to collect the acoustic data on a planar array of receivers from which the range-dependent properties of the sediment can be obtained. Schemes for extracting modal eigenvalues of the propagating modes from data acquired on a curvilinear track with nonuniform grid spacing will be presented and the performance of the schemes evaluated using synthetic and field data.

\section{1:00}

2aUWb9. Bottom reflection phase inversion using modal dispersion curves. B. T. Cox and P. Joseph (Inst. of Sound and Vib. Res., Univ. of Southampton, Southampton SO17 1BJ, UK)

A technique to obtain an estimate of the seabed reflection coefficient phase from modal dispersion curves is presented. The technique is demonstrated on experimental data with a frequency range of 4 to $100 \mathrm{kHz}$ in a 10-cm-deep water waveguide. Thirteen modal dispersion curves, defined as the pressure amplitude as a function of frequency and horizontal wave number, were obtained by measuring the acoustic pressure as a function of range and performing a Hankel transform. Initial attempts have produced estimates of the phase that are in close agreement with an independent estimate obtained by measuring the seabed wave speeds and density. [Work sponsored by EPSRC and DERA (Winfrith).]

\section{1:20}

2aUWb10. Extracting sea-bottom information from reverberation data. Dale D. Ellis (Defence Res. Establishment Atlantic, P.O. Box 1012, Dartmouth, NS B2Y 3Z7, Canada, ellis@drea.dnd.ca) and John R. Preston (The Pennsylvania State Univ., State College, PA 16804)

Reverberation measurements contain information about both the scattering and propagation effects. This paper will discuss our efforts to extract bottom loss and bottom scattering from reverberation data, including at-sea results obtained during three recent Rapid Environmental Assess- ment (REA) trials with the SACLANT Undersea Research Centre. There is often a strong interrelationship between the bottom loss and scattering, and attempts to deal with the uniqueness of the extracted parameters are discussed. Earlier attempts using another simplified, but automated, inverse scheme indicated very good agreement with independently measured bottom loss. In the recent REA trials the initial bottom loss was obtained using some a priori geologic information for the experiment area. Then the bottom parameters were manually adjusted to obtain a good fit to the data from several nearby locations and array headings. The best fits were obtained by first adjusting the bottom loss to fit the rate of decay, and then adjusting the strength of the Lambert coefficient to fit the level.

\section{$11: 40$}

2aUWb11. Estimation of the channel scattering function. Steven Kay (Dept. of Elec. Eng., Univ. of Rhode Island, Kingston, RI 02881, kay@ele.uri.edu) and S. Bradford Doyle (Naval Undersea Warfare Ctr. Div., Newport, RI 02841)

The estimation of the range-Doppler scattering function for an arbitrarily spread (underspread or overspread) channel is addressed. Conventional methods typically require collection of reverberation data from many pings over time to characterize the channel accurately. A new method is proposed based on a minimum mean-square error (MMSE) estimator of a single realization of the channel's time-varying frequency response. Next, a two-dimensional autoregressive spectral estimation technique is used as a scattering function estimator. This approach does not suffer from the usual convolutional smoothing with the signal ambiguity function encountered with Fourier-based methods. The method is compared to traditional methods through simulation. Insight into signal design based on this new method is also provided. [Work supported by NUWC Division, Newport.] 


\title{
Posters from various technical sessions remain on display in the Poster Gallery.
}

Posters from sessions which contain both lecture and poster presentations will be attended by the authors as listed below.

$\begin{array}{ll}\text { 1pNSa12 } & \text { Mirowska, Marianna } \\ \text { 2aPAb13 } & \text { Robsman, Vadim A. } \\ & \text { Robsman, Vadim A. } \\ \text { 2aPAb14 } & \text { Hutchins, D. A. } \\ \text { 2aPAb15 } & \text { Murphy, William J. } \\ \text { 3aNSb12 } & \text { Zannin, Paulo H. T. } \\ \text { 3aNSb13 } & \end{array}$

$$
\text { 2:00-4:00 }
$$

Assessment of low frequency noise in dwellings. New Polish recommendations

Nonlinear acoustics testing of defect for evaluation of risk of insurance of the therman electric power stations

Experience in nonlinear wave testing of constructions inside the earthquake area Experiments in solids using air-coupled ultrasound

Four protectors in search of a rating system

Effects of cup, cushion, headband force, and foam lining on the attenuation of earmuffs

Also, the following poster sessions are scheduled:

Poster Session $2 \mathrm{pSCb}$

Poster Session 2pSPb

TUESDAY AFTERNOON, 16 MARCH 1999

ROOM H105, 1:55 TO 4:00 P.M.

\author{
Session 2pAAa \\ Architectural Acoustics: Worship and Theatre Spaces \\ Emmanuel G. Tzekakis, Cochair \\ Department of Architecture, Aristotle University of Thessaloniki, 540 06, Thessaloniki, Greece \\ Daniel E. Commins, Cochair \\ Commins Acoustics Workshop, 15 rue Laurence Savart, F-75020 Paris, France
}

Chair's Introduction-1:55

Contributed Papers

2:00

2pAAa1. The acoustical design of a new open air theater in Thessaloniki, Greece. Emmanuel G. Tzekakis (Dept. of Architecture, Aristotle Univ. of Thessaloniki, 540 06, Thessaloniki, Greece) and Gottfried Schubert (Consultant in Acoust., Athens, Greece, emil49@compulink.gr)

The international experience in the design of open air theaters is limited. Most new theaters are used exclusively with electroacoustic installations, because they are very large or in very noisy surroundings. As a result, no new architectural or acoustical breakthroughs are available. The new theater reported in this paper is a 5000 seat open air theater, with a shape derived from the ancient Greek prototypes, optimized for best listening conditions without electroacoustic installations. The background noise level reduction and the geometry of the theater were the main concerns of the acoustical design. Modeling tools were used to predict the final acoustical conditions. Measurements made in the almost ready theater indicate that using known and reliable prototypes in a careful way results in high quality acoustical conditions.
2:20

2pAAa2. The ancient Greek theater and its acoustical quality for contemporary performances. Gottfried Schubert (Consultant in Acoust., 22 Apolonos Str., 153 44, Kantza Pallini, Athens, Greece, emil49@compulink.gr) and Emmanuel G. Tzekakis (Aristotle Univ. of Thessaloniki, 540 06, Thessaloniki, Greece)

The acoustical quality of the ancient Greek theater is often described as a phenomenon with mythical dimensions. According to recent experiences with contemporary theater performances at famous historical ancient Greek theaters, the acoustical situation is much more simple and even more problematic than described. To understand the real situation, it is helpful to review the evolution of the ancient theater in terms of construction as well as the theater play as a religious and cultural event. The Dionysus Theater of Athens is one of the most important examples to base this review on. Modern theater festivals also take place in the ancient theater of Epidaurus, which compared to many others, is in a remarkably good condition especially after its restoration. Nevertheless, the scene building is missing and this leads to several acoustical problems. The Epidaurus Theatre is used as an example for the description of the experiences with contemporary performances in ancient Greek theaters. 